\title{
The GALEX/S ${ }^{4}$ G Surface Brightness and Color Profiles Catalog. I. Surface Photometry and Color Gradients of Galaxies
}

\author{
Alexandre Y. K. Bouquin ${ }^{1}\left(\mathbb{D}\right.$, Armando Gil de Paz ${ }^{1}$ (iD, Juan Carlos Muñoz-Mateos ${ }^{2}$, Samuel Boissier ${ }^{3}$ (D), Kartik Sheth ${ }^{4}$ (i), \\ Dennis Zaritsky $^{5}$ (D), Reynier F. Peletier ${ }^{6}$ (D), Johan H. Knapen ${ }^{7,8}$ (D), and Jesús Gallego ${ }^{1}$ (D) \\ ${ }^{1}$ Departamento de Astrofísica y CC. de la Atmósfera, Universidad Complutense de Madrid, E-28040 Madrid, Spain \\ ${ }^{2}$ European Southern Observatory, Casilla 19001, Santiago 19, Chile \\ ${ }^{3}$ Aix Marseille Univ., CNRS, LAM, Laboratoire d'Astrophysique de Marseille, Marseille, France \\ ${ }^{4}$ NASA Headquarters, Washington, DC 20546-0001, USA \\ ${ }^{5}$ Steward Observatory, University of Arizona, 933 North Cherry Avenue, Tucson, AZ 85719, USA \\ ${ }^{6}$ Kapteyn Astronomical Institute, Postbus 800, 9700 AV Groningen, The Netherlands \\ ${ }^{7}$ Instituto de Astrofísica de Canarias, Vía Láctea, S/N, E-38205 La Laguna, Tenerife, Spain \\ ${ }^{8}$ Departamento de Astrofísica, Universidad de La Laguna, E-38205 La Laguna, Tenerife, Spain \\ Received 2017 March 20; revised 2017 October 1; accepted 2017 October 2; published 2018 January 25
}

\begin{abstract}
We present new spatially resolved surface photometry in the far-ultraviolet (FUV) and near-ultraviolet (NUV) from images obtained by the Galaxy Evolution Explorer (GALEX) and IRAC1 $(3.6 \mu \mathrm{m})$ photometry from the Spitzer Survey of Stellar Structure in Galaxies $\left(S^{4} \mathrm{G}\right)$. We analyze the radial surface brightness profiles $\mu_{\mathrm{FUV}}, \mu_{\mathrm{NUV}}$, and $\mu_{[3.6]}$, as well as the radial profiles of (FUV - NUV), (NUV - [3.6]), and (FUV - [3.6]) colors in 1931 nearby galaxies $(z<0.01)$. The analysis of the $3.6 \mu \mathrm{m}$ surface brightness profiles also allows us to separate the bulge and disk components in a quasi-automatic way and to compare their light and color distribution with those predicted by the chemo-spectrophotometric models for the evolution of galaxy disks of Boissier \& Prantzos. The exponential disk component is best isolated by setting an inner radial cutoff and an upper surface brightness limit in stellar mass surface density. The best-fitting models to the measured scale length and central surface brightness values yield distributions of spin and circular velocity within a factor of two of those obtained via direct kinematic measurements. We find that at a surface brightness fainter than $\mu_{[3.6]}=20.89 \mathrm{mag} \mathrm{arcsec}^{-2}$, or below $3 \times 10^{8}$ $M_{\odot} \mathrm{kpc}^{-2}$ in stellar mass surface density, the average specific star formation rate (sSFR) for star-forming and quiescent galaxies remains relatively flat with radius. However, a large fraction of GALEX Green Valley galaxies show a radial decrease in sSFR. This behavior suggests that an outside-in damping mechanism, possibly related to environmental effects, could be testimony of an early evolution of galaxies from the blue sequence of star-forming galaxies toward the red sequence of quiescent galaxies.
\end{abstract}

Key words: catalogs - galaxies: photometry - galaxies: star formation - infrared: galaxies - ultraviolet: galaxies

Supporting material: machine-readable tables

\section{Introduction}

Observing the ultraviolet (UV) part of the electromagnetic spectrum is a direct way to determine the current star formation rate (SFR) in nearby galaxies. The far-ultraviolet (FUV; $\lambda_{\text {eff }}=1516 \AA$ ) band and near-ultraviolet (NUV; $\lambda_{\text {eff }}=$ $2267 \AA$ ) band luminosities are tracers of the most recent star formation in galaxies, up to about 100 million yr, because they are mainly produced by short-lived $\mathrm{O}$ and $\mathrm{B}$ stars and are directly related to the current SFR of galaxies (Kennicutt 1998). Consequently, the FUV observations of nearby galaxies by the Galaxy Evolution Explorer (GALEX) space telescope (Martin et al. 2005) allow us to obtain the amount of stars formed in nearby disk galaxies and dwarfs. In the last two decades, restframe UV observations have also been used to analyze the evolution of the SFR throughout the history of the universe (see the review by Madau \& Dickinson 2014). However, a detailed analysis of the spatial distribution of the SFR, starting from local galaxies, is needed if we want to understand the origin and mechanisms involved in the evolution of the SFR in general and the observed decay in the SFR since $z \sim 1$.

In spite of the rather quick evolution since $z=1$, many galaxies have kept forming stars until now, some of them vigorously at all galactocentric distances (the so-called extended UV-disk galaxies constitute a prime example in that regard; Gil de Paz et al. 2005, 2007; Thilker et al. 2005, 2007). However, many others (especially massive ones, but not exclusively) have had their star formation quenched or at least damped, in the sense that their star formation substantially decreased (and not in the sense that gas has been exhausted) at different epochs and galactocentric distances. Our ultimate goal is to address the study of these objects using multiwavelength surface photometry combined for an unprecedented large sample of galaxies in the local universe. The sensitivity of the UV emission to even small amounts of star formation allows us to identify objects that are going through a transition phase and to determine whether this transition occurs at all radii at the same time or in an outside-in or inside-out fashion. However, in order to relate the current SFR with that having occurred in the past, the distribution of the UV emission must be compared with that of the galaxy's stellar mass all the way to the very faint outskirts of galaxies. Deep rest-frame nearinfrared imaging data are key in that regard, such as those provided by the Infrared Array Camera (IRAC) onboard the Spitzer satellite in the case of nearby galaxies and soon by the James Webb Space Telescope at intermediate-to-high redshifts. These observations allow us to probe the radial variations of the SFR in relation to the stellar mass surface density. Spatially resolved radial color profiles are a powerful diagnostic tool to 
gain insight into the relative number of young to old stars. However, most of the results obtained to date have focused on the global properties of galaxies, even in nearby galaxies. There are noticeable exceptions, such as the work of Muñoz-Mateos et al. (2011) and Pezzulli et al. (2015), but usually for relatively small samples (75 and 35 nearby spiral galaxies, respectively, in these examples).

Studies of the integrated (NUV $-r$ ) versus $r$ colormagnitude diagram for nearby galaxies have revealed a clear bimodal distribution (e.g., Martin et al. 2007; Wyder et al. 2007): quiescent, early-type galaxies (ETGs) are seen to form a "red sequence," whereas actively star-forming late-type galaxies are seen to form a "blue sequence." This has been seen both in the field galaxy population and in nearby clusters such as Virgo (Boselli et al. 2005). A recent study of the so-called "Green Valley Galaxies" (GVG) using the Sloan Digital Sky Survey (SDSS) data and defined in the $(u-r)$ color-mass diagram by Schawinski et al. (2014) shows that GVGs span a wide range of colors and masses. As pointed out by Schawinski et al. (2014), using UV-optical bands helps constrain the star formation quenching timescale. We have shown in Bouquin et al. (2015) that using the (FUV - NUV) versus (NUV - $3.6 \mu \mathrm{m}$ ) color-color diagram constrains the star formation quenching timescale to be less than $1 \mathrm{Gyr}$.

Integrated color-color diagrams have been widely used in the past to investigate integrated properties of galaxies. For example, the (FUV - NUV) versus (NUV - K) (Gil de Paz et al. 2007) or the (FUV - NUV) versus (NUV - [3.6]) (Bouquin et al. 2015) color-color diagrams can well separate the star-forming galaxies from quiescent galaxies. Bouquin et al. (2015) showed that the combination of UV and IR reveals a better sequential distribution than the "classical" optical-IR color-color diagrams, especially for star-forming (Blue Clouds) systems. These color-color diagrams separate nearby galaxies into a very narrow sequence of star-forming galaxies populated mostly by late-type galaxies, which we dubbed the GALEX Blue Sequence (GBS), and a broader sequence, the GALEX Red Sequence (GRS), where quiescent galaxies such as ETGs are distributed.

The above studies utilize global properties of galaxies but do not assess the distribution of star formation within galaxies. It is of crucial importance that we understand how star formation is happening within nearby galaxies, where the active zones are, and, based on that information, determine what mechanism(s) are in effect for activating or suppressing star formation in order to compare star formation of galaxies at high redshift. Looking at the spatially resolved radial profiles is of the utmost importance, as it can give us insight into galaxy disk growth and how quenching takes places (from the inside out or from the outside in).

Recently, a deep infrared survey of nearby galaxies, the Spitzer Survey of Stellar Structure in Galaxies $\left(S^{4} G\right.$; Sheth et al. 2010), has been undertaken using the IRAC onboard the Spitzer Space Telescope. We used the $\sim 2300 \mathrm{~S}^{4} \mathrm{G}$ galaxies as our base sample, complemented it with the publicly available GALEX counterparts (GR6/7) for those galaxies, and performed new FUV (1350-1750 ̊) and NUV (1750-2800 $)$ photometry. We obtained surface brightness profiles in FUV and NUV, as well as (FUV - NUV) color profiles, for 1931 nearby galaxies up to $40 \mathrm{Mpc}$. These data provide both broad wavelength coverage and good physical spatial resolution. At the median distance of the survey, $23 \mathrm{Mpc}$, a GALEX point spread function (PSF) of $6^{\prime \prime}$ corresponds to $\sim 700 \mathrm{pc}$ (but varies from 12 to $2737 \mathrm{pc}$ for ESO $245-007$ at $0.42 \mathrm{Mpc}$ to PGC 040552 at $94.1 \mathrm{Mpc}){ }^{9}$

This paper follows a classical approach in its structure, starting with an overview of the criteria used to constrain the initial sample of galaxies (Sections 2.1, 2.2). Once the sample is defined, we describe the reduction processes to obtain our science-ready products (Sections 3, 3.1) and the analysis performed (Sections 3.2, 3.3). Results and discussion of that analysis are described in the section that follows (Section 4). Then, we also show in Sections 5 and 5.1 and a study on obtaining the circular velocities and spin parameters from the models of Boissier \& Prantzos (2000; hereafter BP00)and how they compare to the observed values (Section 5.2). This is followed by a discussion of the results of this work in Sections 6 and 6.1-6.3. Finally, the summary and conclusions are in Section 7. The derivation of stellar mass surface density from the $3.6 \mu \mathrm{m}$ surface brightness is included in Appendix A, followed by the derivation of the specific star formation rate (sSFR) from the (FUV - [3.6]) color in Appendix B.

We assume a standard $\Lambda$ CDM cosmology with $H_{0}=$ $75 \mathrm{~km} \mathrm{~s}^{-1} \mathrm{Mpc}^{-1}$, and all magnitudes throughout this paper are given in the $\mathrm{AB}$ system unless stated otherwise.

\section{Sample}

In this section, we briefly describe the criteria used to select the $S^{4} \mathrm{G}$ sample (Section 2.1) and, in more detail, the method of retrieval of the cross-matched UV data (Section 2.2). However, the reader is referred to Sheth et al. (2010) for more details about the $S^{4} \mathrm{G}$ sample selection. This study is uniquely based on imaging data.

\section{1. $S^{4} G$}

The $S^{4} G$ galaxy sample is a deep infrared survey of a (mainly) volume-limited sample of nearby galaxies within $d<40 \mathrm{Mpc}$ observed at 3.6 and $4.5 \mu \mathrm{m}$ with the IRAC (Fazio et al. 2004; see Sheth et al. 2010 for a full description of the survey). Additional selection criteria are size-limited with $D_{25}>1^{\prime}$, magnitude-limited in $B$-band (Vega) $<15.5 \mathrm{mag}$, and above and below the Galactic plane $|b|>30^{\circ}$. The total sample size is 2352 galaxies. A follow-up survey was done to include more ETGs, but those data are not included in this catalog.

A multiwavelength analysis of the $S^{4} G$ sample has since been carried out as part of the Detailed Anatomy of Galaxies (DAGAL) project, and it is now complemented with FUV and NUV data from GALEX (see also Zaritsky et al. 2014b, 2015 and Bouquin et al. 2015 for preliminary analyses of the UVobserved sample), ugriz images from SDSS, and various other data, such as H I data cubes (see Ponomareva et al. 2016) and $\mathrm{H} \alpha$ images (e.g., Knapen et al. 2004; Erroz-Ferrer et al. 2012). Additional analyses and catalogs, such as a classical morphological classification (Buta et al. 2015), bulge/disk decomposition (from the $\mathrm{S}^{4} \mathrm{G}$ P4 pipeline; Salo et al. 2015), catalog of morphological features (Herrera-Endoqui et al. 2015), and stellar mass catalog (P5; Querejeta et al. 2015), have also been produced and are publicly available online. ${ }^{10}$ Much more

\footnotetext{
9 One of the sample selection criteria uses the distance inferred from the radial velocity measurements from HI observations, whereas here we use the redshift-independent distance, hence the discrepancy.

${ }^{10}$ http://www.astro.rug.nl/ dagal/
} 
detailed analyses of specific subsamples within $\mathrm{S}^{4} \mathrm{G}$ are also available elsewhere, such as a catalog of structural parameters from BUDDA decomposition (de Souza et al. 2004; Gadotti 2008) of $3.6 \mu \mathrm{m}$ images (Kim et al. 2016) and $\mathrm{H} \alpha$ kinematic studies of the inner regions (Erroz-Ferrer et al. 2016).

In this paper, we have used the surface photometry at $3.6 \mu \mathrm{m}$ (IRAC1) measurements from the output of pipeline 3 (P3) of the $S^{4} G$ sample (see Muñoz-Mateos et al. 2015 for a detailed description of the $\mathrm{S}^{4} \mathrm{GP} 3$ treatment). We have collected these data from the IRSA database ${ }^{11}$ via their dedicated website. We only used the $3.6 \mu \mathrm{m}$ surface photometry performed with a fixed aperture geometry (file names of the form *.1fx2a_noclean_fin.dat) where the center, position angle, and ellipticity are all kept fixed and only the aperture radius is increased by radial increments of $2^{\prime \prime}$ along the semimajor axis. Subsequent mentions of $\mu_{[3.6]}$ correspond to the aperture-corrected surface brightness (columns SB_COR and its error ESB_COR, as well as the cumulative magnitude TMAG_COR and its error ETMAG_COR) found in these publicly available data. Since our GALEX photometry is performed every $6^{\prime \prime}$ in major-axis radius steps, we only use the data outputs obtained at the same step values for the $3.6 \mu \mathrm{m}$ photometry.

Table 1 shows the first galaxies of our GALEX/S ${ }^{4} \mathrm{G}$ sample sorted by R.A. and lists the FUV and NUV asymptotic magnitudes obtained for our sample along the $3.6 \mu \mathrm{m}$ asymptotic magnitudes obtained by Muñoz-Mateos et al. (2015). The complete table, with additional columns such as which GALEX tiles were used, is publicly available online through VizieR (Ochsenbein et al. 2000).

\subsection{GALEX Counterparts}

We gathered all available GALEX FUV and NUV images and related data products for $1931 \mathrm{~S}^{4} \mathrm{G}$ galaxies that had been observed in at least one of these two UV bands. Over 200 galaxies do not have GALEX data at all. We obtained the original GALEX data using the GALEXview ${ }^{12}$ tool. Priority was given to galaxies that have both FUV and NUV images, with the longest FUV exposure time. If this condition was met for several product tiles (including a very similar exposure time to the NUV in the FUV), we chose the one where the target galaxy was best centered in the field-of-view (FOV). We collected imaging data from all kinds of surveys, such as the All-sky Imaging Survey, Medium Imaging Survey, Deep Imaging Survey, and Nearby Galaxy Survey, as well as from Guest Investigator (GIs/GIIs) Programs.

The collected data, once processed, yielded a total of 1931 galaxies with both FUV and NUV photometry available. We call this sample, derived from the $\mathrm{S}^{4} \mathrm{G}$ and having FUV, NUV, and IRAC1 $3.6 \mu \mathrm{m}$ photometry, the $G A L E X / \mathrm{S}^{4} \mathrm{G}$ sample. We compare the $\mathrm{S}^{4} \mathrm{G}$ sample and the GALEX/S $\mathrm{S}^{4} \mathrm{G}$ sample in Figure 1. The distributions of distances, apparent $B$-band magnitudes, and morphological types of the two samples and the distribution of the integrated (FUV - NUV) colors of the final $G A L E X / \mathrm{S}^{4} \mathrm{G}$ sample are shown. Demographics are shown in Table 2. Our GALEX $/ \mathrm{S}^{4} \mathrm{G}$ sample is clearly representative of the whole $S^{4} G$ sample with only minor differences in the case of the absolute magnitude distribution. Note that every $\mathrm{S}^{4} \mathrm{G}$ galaxy targeted with GALEX was detected and its UV fluxes measured.

\footnotetext{
${ }^{11}$ http://irsa.ipac.caltech.edu/data/SPITZER/S4G/

12 http://galex.stsci.edu/GalexView/
}

We also subdivided the $G A L E X / \mathrm{S}^{4} \mathrm{G}$ sample into three subsamples. This was done according to the preliminary analysis of the UV-to-IR photometry of Bouquin et al. (2015), where we presented our sample of 1931 galaxies with their asymptotic magnitudes plotted on an (FUV - NUV) versus (NUV - [3.6]) color-color diagram. From this integrated color-color diagram, we were able to select three subsamples of galaxies, namely the GBS, GRS, and GALEX Green Valley (GGV) galaxies, which were defined as follows:

$$
\text { GBS: } 0.12 x+0.16-2 \sigma_{\mathrm{GBS}} \leqslant y \leqslant 0.12 x+0.16+2 \sigma_{\mathrm{GBS}} \text {, }
$$

$$
\begin{aligned}
\mathrm{GRS}: & -0.23 y+5.63-1 \sigma_{\mathrm{GRS}} \leqslant x \leqslant-0.23 y \\
& +5.63+1 \sigma_{\mathrm{GRS}}
\end{aligned}
$$

and

$$
\begin{aligned}
\mathrm{GGV}: y>0.12 x & +0.16+2 \sigma_{\mathrm{GBS}} \text { and } x<-0.23 y \\
& +5.63-1 \sigma_{\mathrm{GRS}},
\end{aligned}
$$

where $x=(\mathrm{NUV}-[3.6]), y=(\mathrm{FUV}-\mathrm{NUV}), \sigma_{\mathrm{GBS}}=0.20$, and $\sigma_{\mathrm{GRS}}=0.45$. Both the GBS and GRS are defined to be stripes defined between two parallel lines (Equations (1) and (2)). Note that the GRS equations are expressed in the $y x$ space. The GGV is the region bluer in (NUV - [3.6]) than the GRS but redder in (FUV - NUV) than the GBS (Equation (3)).

The GBS is populated by star-forming galaxies and mostly late-type galaxies, while the GRS is populated by redder systems that lack star formation (quiescent) and are passively evolving or where only low levels of residual star formation are present (e.g., Boselli et al. 2005; Y1ld1z et al. 2017) and that are, morphologically speaking, mostly ETGs. The GGV galaxies are found between the GBS and the GRS in this UV-to-IR color-color plane, and they are special in the sense that these galaxies can be seen to have decreased star formation activity in recent epochs. Hence, their (FUV - NUV) colors are redder than in GBS galaxies, and their (NUV - [3.6]) colors are bluer than in GRS galaxies. However, it should be noted that we do not exclude the possibility that this GGV population could represent GRS galaxies that are being rejuvenated, thus showing a bluing (FUV - NUV) color. The important point here is that the quick response of the (FUV - NUV) color to even small amounts of recent star formation, coupled with the tightness of the GBS, allows identifying galaxies that are just starting to experience these quenching or rejuvenating events. See Bouquin et al. (2015) for further details.

\section{Analysis}

In this section, we describe our method of analysis of the NIR and UV imaging data acquired by Spitzer IRAC1 and $G A L E X$ in order to obtain $3.6 \mu \mathrm{m}$, FUV, and NUV surface photometry. The acquirement of the $3.6 \mu \mathrm{m}$ surface photometry is not described, here as it is already explained in Muñoz-Mateos et al. (2015), and we only focus on the FUV and NUV surface photometry in this article (Section 3.1). We also performed a radial normalization of the $3.6 \mu \mathrm{m}$ radial profiles (Section 3.2) and constructed the (FUV - NUV), (FUV - [3.6]), and (NUV - [3.6]) color profiles (Section 3.3). 
Table 1

The GALEX/S ${ }^{4}$ G Sample

\begin{tabular}{|c|c|c|c|c|c|c|c|c|}
\hline Name $^{a}$ & $\begin{array}{l}\begin{array}{l}\text { R.A. }^{\mathrm{b}} \\
\text { (deg) }\end{array}\end{array}$ & $\begin{array}{l}\begin{array}{c}\text { Decl. }^{\mathrm{c}} \\
(\mathrm{deg})\end{array} \\
\end{array}$ & $T^{\mathrm{d}}$ & $\begin{array}{c}\text { Distance }^{\mathrm{e}} \\
(\mathrm{Mpc})\end{array}$ & $\begin{array}{c}\mathrm{FUV}^{\mathrm{f}} \\
(\mathrm{AB} \mathrm{mag})\end{array}$ & $\begin{array}{c}\mathrm{NUV}^{\mathrm{g}} \\
(\mathrm{AB} \mathrm{mag})\end{array}$ & $\begin{array}{c}M_{3.6}{ }^{\mathrm{h}} \\
(\mathrm{AB} \mathrm{mag})\end{array}$ & Group ID \\
\hline UGC 00017 & 0.929725 & 15.218985 & 9.1 & $13.0 \pm \ldots$ & $16.86 \pm 0.08$ & $16.59 \pm 0.02$ & $14.880 \pm 0.006$ & 1211 \\
\hline ESO 409-015 & 1.383640 & -28.099908 & 5.4 & $9.8 \pm \ldots$ & $15.94 \pm 0.01$ & $15.86 \pm 0.01$ & $15.873 \pm 0.001$ & 0 \\
\hline ESO 293-034 & 1.583550 & -41.497280 & 6.2 & $18.3 \pm \ldots$ & $14.77 \pm 0.01$ & $14.38 \pm 0.01$ & $11.612 \pm 0.001$ & 0 \\
\hline NGC 0007 & 2.087407 & -29.914812 & 4.8 & $21.9 \pm 1.6$ & $15.48 \pm 0.01$ & $15.18 \pm 0.01$ & $14.021 \pm 0.002$ & 1096 \\
\hline IC 1532 & 2.468434 & -64.372169 & 4.0 & $28.7 \pm 5.3$ & $16.74 \pm 0.08$ & $16.38 \pm 0.01$ & $14.590 \pm 0.004$ & 1031 \\
\hline NGC 0024 & 2.484438 & -24.964018 & 5.1 & $6.9 \pm 2.8$ & $14.11 \pm 0.01$ & $13.79 \pm 0.01$ & $11.492 \pm 0.001$ & 355 \\
\hline ESO 293-045 & 2.853125 & -41.398099 & 7.8 & $27.9 \pm 5.5$ & $16.27 \pm 0.01$ & $16.11 \pm 0.01$ & $15.784 \pm 0.007$ & 0 \\
\hline UGC 00122 & 3.323550 & 17.029280 & 9.6 & $11.6 \pm 0.7$ & $16.05 \pm 0.01$ & $15.89 \pm 0.01$ & $15.815 \pm 0.029$ & 0 \\
\hline UGC 00132 & 3.503175 & 12.963801 & 7.9 & $22.4 \pm \ldots$ & $17.21 \pm 0.02$ & $16.69 \pm 0.05$ & $14.585 \pm 0.001$ & 0 \\
\hline NGC 0059 & 3.854846 & -21.444339 & -2.9 & $4.9 \pm 0.6$ & $16.10 \pm 0.01$ & $15.35 \pm 0.01$ & $12.749 \pm 0.001$ & 0 \\
\hline UGC 00156 & 4.199970 & 12.350260 & 9.8 & $15.9 \pm \ldots$ & $16.60 \pm 0.07$ & $15.73 \pm 0.07$ & $14.176 \pm 0.001$ & 0 \\
\hline NGC 0063 & 4.439552 & 11.450338 & -3.4 & $18.8 \pm 0.2$ & $16.81 \pm 0.03$ & $15.61 \pm 0.02$ & $11.838 \pm 0.001$ & 1213 \\
\hline ESO 539-007 & 4.701543 & -19.007968 & 8.7 & $25.6 \pm \ldots$ & $16.27 \pm 0.07$ & $16.05 \pm 0.03$ & $15.256 \pm 0.011$ & 0 \\
\hline ESO 150-005 & 5.607727 & -53.648004 & 7.8 & $15.2 \pm 2.2$ & $15.48 \pm 0.01$ & $15.26 \pm 0.01$ & $14.083 \pm 0.006$ & 0 \\
\hline NGC 0100 & 6.011113 & 16.486026 & 5.9 & $16.4 \pm 3.1$ & $15.79 \pm 0.04$ & $15.32 \pm 0.01$ & $13.002 \pm 0.002$ & 1214 \\
\hline NGC 0115 & 6.692700 & -33.677098 & 3.9 & $30.7 \pm 5.3$ & $15.16 \pm 0.01$ & $14.91 \pm 0.01$ & $13.752 \pm 0.001$ & 1097 \\
\hline UGC 00260 & 6.762137 & 11.583803 & 5.8 & $32.3 \pm 2.3$ & $15.36 \pm 0.01$ & $15.04 \pm 0.01$ & $12.767 \pm 0.001$ & 1188 \\
\hline ESO 410-012 & 7.073298 & -27.982521 & 4.6 & $20.6 \pm \ldots$ & $17.44 \pm 0.01$ & $17.18 \pm 0.01$ & $16.736 \pm 0.006$ & 0 \\
\hline UGC 00290 & 7.284883 & 15.899069 & 9.5 & $9.0 \pm 0.2$ & $17.66 \pm 0.21$ & $17.36 \pm 0.08$ & $16.412 \pm 0.005$ & 0 \\
\hline NGC 0131 & 7.410483 & -33.259902 & 3.0 & $18.8 \pm \ldots$ & $16.08 \pm 0.01$ & $15.65 \pm 0.01$ & $13.036 \pm 0.002$ & 0 \\
\hline UGC 00313 & 7.858420 & 6.206820 & 4.3 & $27.8 \pm \ldots$ & $16.78 \pm 0.11$ & $16.34 \pm 0.04$ & $13.976 \pm 0.004$ & 0 \\
\hline ESO 079-003 & 8.009728 & -64.253213 & 3.1 & $39.0 \pm 4.1$ & $16.66 \pm 0.02$ & $16.14 \pm 0.03$ & $11.604 \pm 0.001$ & 0 \\
\hline UGC 00320 & 8.128720 & 2.574640 & 6.1 & $40.8 \pm 4.7$ & $17.36 \pm 0.01$ & $17.04 \pm 0.01$ & $15.949 \pm 0.001$ & 0 \\
\hline IC 1553 & 8.167184 & -25.607556 & 7.0 & $33.4 \pm 1.6$ & $16.17 \pm 0.02$ & $15.87 \pm 0.01$ & $12.970 \pm 0.001$ & 1300 \\
\hline ESO 410-018 & 8.545903 & -30.774519 & 8.9 & $19.0 \pm \ldots$ & $15.39 \pm 0.01$ & $15.21 \pm 0.01$ & $14.536 \pm 0.057$ & 0 \\
\hline NGC 0150 & 8.564448 & -27.803522 & 3.4 & $21.0 \pm 3.3$ & $14.19 \pm 0.01$ & $13.86 \pm 0.01$ & $10.918 \pm 0.001$ & 1100 \\
\hline NGC 0148 & 8.564559 & -31.785999 & -2.0 & $18.4 \pm \ldots$ & $19.37 \pm 0.66$ & $17.79 \pm 0.12$ & $11.744 \pm 0.001$ & 0 \\
\hline IC 1555 & 8.636397 & -30.017818 & 7.0 & $23.1 \pm 2.0$ & $15.94 \pm 0.01$ & $15.52 \pm 0.01$ & $14.438 \pm 0.001$ & 1096 \\
\hline NGC 0157 & 8.694906 & -8.396344 & 4.0 & $19.5 \pm 5.4$ & $13.59 \pm 0.01$ & $12.96 \pm 0.01$ & $10.066 \pm 0.001$ & 1105 \\
\hline IC 1558 & 8.946172 & -25.374404 & 9.0 & $13.7 \pm 4.6$ & $14.73 \pm 0.01$ & $14.43 \pm 0.01$ & $13.337 \pm 0.002$ & 1100 \\
\hline NGC 0178 & 9.784857 & -14.172626 & 8.7 & $18.4 \pm \ldots$ & $14.16 \pm 0.01$ & $13.99 \pm 0.01$ & $13.193 \pm 0.001$ & 0 \\
\hline NGC 0210 & 10.145717 & -13.872773 & 3.1 & $21.0 \pm 1.3$ & $13.99 \pm 0.08$ & $13.76 \pm 0.01$ & $10.792 \pm 0.001$ & 1102 \\
\hline ESO 079-005 & 10.182495 & -63.441987 & 7.0 & $23.5 \pm 2.8$ & $15.28 \pm 0.01$ & $14.97 \pm 0.01$ & $13.866 \pm 0.004$ & 1032 \\
\hline NGC 0216 & 10.363123 & -21.044899 & -1.9 & $19.1 \pm \ldots$ & $15.54 \pm 0.01$ & $15.10 \pm 0.01$ & $13.059 \pm 0.004$ & 0 \\
\hline PGC 002492 & 10.439405 & -16.860757 & 2.0 & $20.7 \pm \ldots$ & $15.74 \pm 0.02$ & $15.50 \pm 0.01$ & $14.155 \pm 0.006$ & 0 \\
\hline IC 1574 & 10.765448 & -22.245836 & 9.9 & $4.8 \pm 0.2$ & $16.57 \pm 0.01$ & $16.09 \pm 0.01$ & $14.749 \pm 0.017$ & 355 \\
\hline NGC 0244 & 11.443430 & -15.596570 & -2.0 & $11.6 \pm \ldots$ & $15.19 \pm 0.01$ & $14.94 \pm 0.01$ & $13.593 \pm 0.003$ & 0 \\
\hline PGC 002689 & 11.515689 & -11.506472 & 8.8 & $20.2 \pm \ldots$ & $15.37 \pm 0.03$ & $15.21 \pm 0.02$ & $14.693 \pm 0.009$ & 0 \\
\hline UGC 00477 & 11.554634 & 19.489885 & 7.9 & $35.8 \pm 0.4$ & $15.86 \pm 0.01$ & $15.66 \pm 0.01$ & $14.328 \pm 0.002$ & 1294 \\
\hline ESO 411-013 & 11.776317 & -31.581403 & 9.0 & $23.5 \pm \ldots$ & $17.87 \pm 0.25$ & $17.36 \pm 0.03$ & $16.037 \pm 0.002$ & 0 \\
\hline NGC 0247 & 11.785305 & -20.760176 & 6.9 & $3.6 \pm 0.5$ & $11.42 \pm 0.02$ & $11.12 \pm 0.02$ & $9.135 \pm 0.001$ & 233 \\
\hline NGC 0254 & 11.865155 & -31.421775 & -1.2 & $17.1 \pm \ldots$ & $17.71 \pm 0.15$ & $16.39 \pm 0.03$ & $11.387 \pm 0.001$ & 0 \\
\hline NGC 0255 & 11.946929 & -11.468734 & 4.1 & $20.0 \pm \ldots$ & $13.98 \pm 0.01$ & $13.75 \pm 0.02$ & $12.252 \pm 0.002$ & 0 \\
\hline PGC 002805 & 11.948177 & -9.899568 & 6.7 & $16.4 \pm 0.5$ & $15.61 \pm 0.01$ & $15.32 \pm 0.01$ & $14.624 \pm 0.005$ & 1101 \\
\hline ESO 540-031 & 12.457500 & -21.012730 & 9.8 & $3.4 \pm 0.2$ & $16.85 \pm 0.01$ & $16.59 \pm 0.02$ & $16.189 \pm 0.048$ & 233 \\
\hline ESO 079-007 & 12.517568 & -66.552204 & 4.0 & $25.2 \pm 4.5$ & $15.21 \pm 0.04$ & $14.94 \pm 0.01$ & $13.570 \pm 0.001$ & 0 \\
\hline NGC 0274 & 12.757695 & -7.056978 & -2.8 & $20.3 \pm 1.5$ & $14.52 \pm 0.01$ & $14.13 \pm 0.01$ & $12.091 \pm 0.001$ & 1103 \\
\hline NGC 0275 & 12.768555 & -7.065730 & 6.0 & $21.9 \pm \ldots$ & $14.50 \pm 0.01$ & $14.15 \pm 0.01$ & $12.284 \pm 0.001$ & 0 \\
\hline PGC 003062 & 13.072022 & -3.966015 & 6.8 & $18.8 \pm \ldots$ & $17.04 \pm 0.20$ & $16.59 \pm 0.02$ & $15.291 \pm 0.009$ & 0 \\
\hline NGC 0289 & 13.176101 & -31.205822 & 4.0 & $22.8 \pm 4.1$ & $13.32 \pm 0.05$ & $13.15 \pm 0.06$ & $10.522 \pm 0.003$ & 1098 \\
\hline
\end{tabular}

Notes. Our sample of 1931 galaxies, sorted by R.A.

${ }_{b}^{\text {a }}$ Same as the $\mathrm{S}^{4} \mathrm{G}$ nomenclature.

b R.A. in degrees and in epoch J2000.0.

c Decl. in degrees and in epoch J2000.0.

${ }^{\mathrm{d}}$ Numerical morphological type from RC2.

e Mean redshift-independent distance measurements with $1 \sigma$ uncertainty from NED if available; see text for details.

f Total FUV apparent magnitude with $1 \sigma$ uncertainty. These uncertainties do not include zero-point errors or errors associated with the misidentification of background or foreground sources.

g Total apparent magnitude with $1 \sigma$ uncertainty. Also see above.

h Total $3.6 \mu \mathrm{m}$ apparent magnitude from IRAC1 photometry and $1 \sigma$ uncertainty (Muñoz-Mateos et al. 2015).

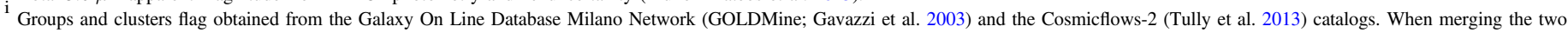

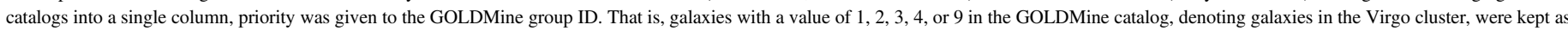

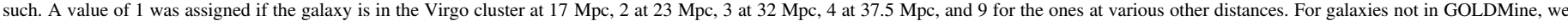

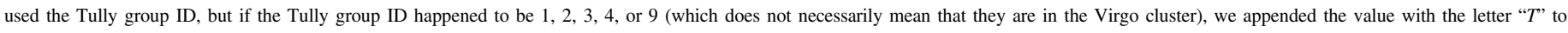

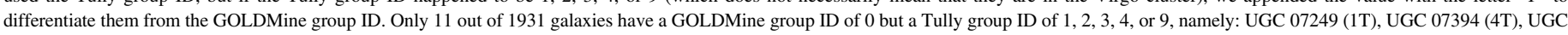

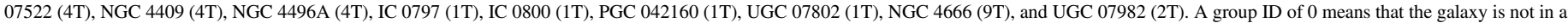
group in either catalog.

(This table is available in its entirety in machine-readable form.) 

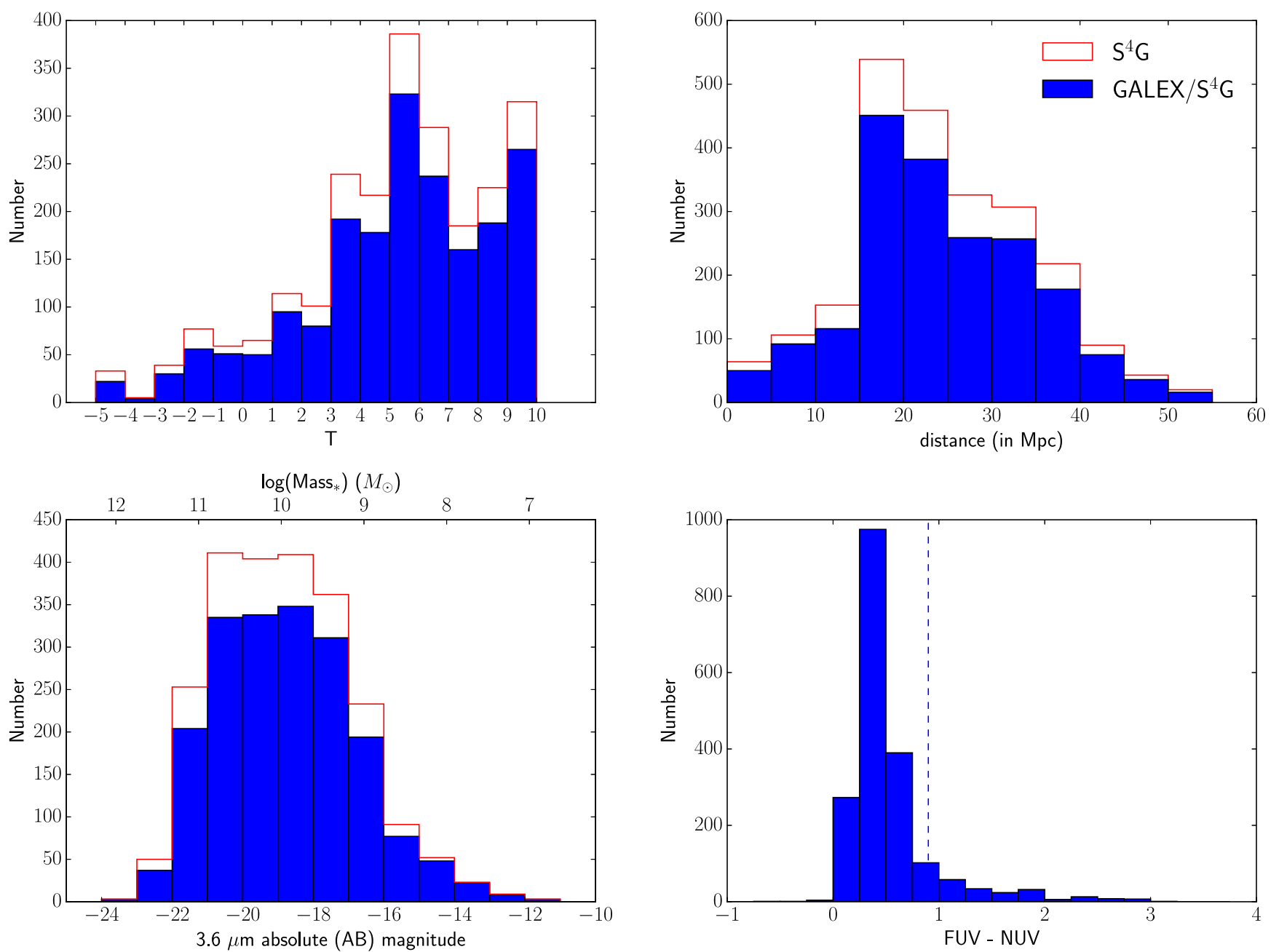

Figure 1. Comparisons of the distributions of the $\mathrm{S}^{4} \mathrm{G}$ sample (open bars) with the $G A L E X / \mathrm{S}^{4} \mathrm{G}$ subsample (filled bars). The distributions of numerical morphological types $(T)$ (binning $=1$; top left), distances in $\mathrm{Mpc}$ (binning $=5 \mathrm{Mpc}$; top right), $3.6 \mu \mathrm{m}$ absolute $\mathrm{AB}$ magnitudes (binning $=1 \mathrm{mag}$; bottom left), and $\left(\right.$ FUV - NUV) color (binning $=0.25 \mathrm{mag}$; bottom right) of both samples are shown. The $\mathrm{S}^{4} \mathrm{G}$ sample is comprised of 2352 galaxies, and the $G A L E X / \mathrm{S}^{4} \mathrm{G}$ subsample is comprised of 1931 galaxies. The $3.6 \mu \mathrm{m}$ absolute magnitude is accompanied by the logarithm of the stellar mass in the top $x$-axis, computed using Equation (12) in Appendix A. The vertical line at (FUV - NUV) $=0.9$ corresponds to the value used in Gil de Paz et al. (2007) to broadly separate early- and late-type galaxies.

\subsection{UV Surface Photometry and Asymptotic Magnitudes}

We obtained spatially resolved FUV and NUV surface photometry, as well as asymptotic magnitudes, for the 1931 galaxies in our $G A L E X / \mathrm{S}^{4} \mathrm{G}$ sample. Three types of GALEX data products were collected from the database:

1. the intensity maps in FUV ('fd-int.fits) and NUV ("ndint.fits),

2. the high-resolution relative response maps in FUV ( ${ }^{*} \mathrm{fd}-$ rrhr.fits) and NUV ( ${ }^{*}$ nd-rrhr.fits), and

3. the object masks in FUV ( ${ }^{*}$ fd-objmask.fits) and NUV ( ${ }^{*}$ nd-objmask.fits).

Once all data were gathered, we proceeded to reduce and analyze our GALEX UV sample in the same manner as in Gil de Paz et al. (2007).

First, a sky value was measured from the surroundings of the target galaxy. This was followed by the preparation of a mask in two steps. In the first step of the masking process, we masked automatically unresolved sources that had (FUV - NUV) colors redder than $1 \mathrm{mag}$, which masks out most foreground stars. This was followed by careful visual checks verifying each and every single galaxy and carefully editing the masks one by one by manually adding or removing masks, since the automatization could (a) falsely detect bulges, (b) fail to select companions, and (c) foreground blue stars, all for the benefit of preserving very blue star-forming regions, especially those in the outskirts of disk galaxies. We unmasked all affected bulges and tried to include as many star-forming regions that were falsely masked, while foreground stars were masked out as much as possible. In the process, we also generated FUV+NUV red giant branch (RGB) images for each galaxy that were used during the manual masking process in order to have an educated guess on any potential masking failure encountered. Although great care had been taken during this masking process, it should be noted that in some cases (e.g., merging galaxies, galaxies with bright stars nearby, objects at the edge of the FOV, bad image quality) difficult choices had to be made. We acknowledge that in those cases (less than a few percent) the values obtained may differ from those obtained by other authors (our masks can be provided on demand). Errors associated with these effects cannot be accounted for and are not included in Table 1. 
Table 2

Galaxy Sample Demographics

\begin{tabular}{|c|c|c|c|}
\hline Galaxy Sample $^{\mathrm{a}}$ & & $\overline{N N}$ & 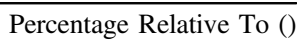 \\
\hline $\begin{array}{l}\mathrm{S}^{4} \mathrm{G} \\
G A L E X / \mathrm{S}^{4} \mathrm{G}\end{array}$ & & $\begin{array}{l}2352 \\
1931\end{array}$ & $\begin{array}{l}100 \% \\
82.1 \%\left(\mathrm{~S}^{4} \mathrm{G}\right)\end{array}$ \\
\hline $\begin{array}{l}\text { GBS } \\
\text { GGV } \\
\text { GRS } \\
\text { Others }\end{array}$ & & $\begin{array}{r}1753 \\
70 \\
79 \\
29\end{array}$ & $\begin{array}{l}90.8 \%\left(G A L E X / \mathrm{S}^{4} \mathrm{G}\right) \\
3.6 \% \ldots \\
4.1 \% \ldots \\
1.5 \% \ldots\end{array}$ \\
\hline ETGs & $\begin{array}{c}\text { E } \\
\text { E-S0 }\end{array}$ & $\begin{array}{l}24 \\
23\end{array}$ & $\begin{array}{l}1.2 \%\left(G A L E X / \mathrm{S}^{4} \mathrm{G}\right) \\
1.2 \% \ldots\end{array}$ \\
\hline ETDGs & $\begin{array}{l}\text { S0 } \\
\text { S0-a } \\
\text { Sa }\end{array}$ & $\begin{array}{r}51 \\
103 \\
175\end{array}$ & $\begin{array}{l}2.6 \% \ldots \\
5.3 \% \ldots \\
9.1 \% \ldots\end{array}$ \\
\hline LTGs & $\begin{array}{l}\mathrm{Sb} \\
\mathrm{Sc} \\
\mathrm{Sd} \\
\mathrm{Sm} \\
\mathrm{Irr}\end{array}$ & $\begin{array}{l}340 \\
669 \\
168 \\
192 \\
186\end{array}$ & $\begin{array}{r}17.6 \% \ldots \\
34.7 \% \ldots \\
8.7 \% \ldots \\
9.9 \% \ldots \\
9.6 \% \ldots\end{array}$ \\
\hline
\end{tabular}

Note.

${ }^{\mathrm{a}}$ Name of the samples. GBS $=$ GALEX Blue Sequence, GGV $=$ GALEX Green Valley, GRS $=$ GALEX Red Sequence, ETGs = early-type galaxies, ETDGs $=$ early-type disk galaxies, LTGs = late-type galaxies. RC2 morphological types were obtained from HyperLeda.

Then, surface brightnesses were measured by averaging over annuli with the same position angle (PA) and ellipticity $(\epsilon)$ as those used in the analysis of the $S^{4} \mathrm{G}$ sample IRAC data. We used a step in major-axis radius of $6^{\prime \prime}$ and integrated over a width of $\pm 3^{\prime \prime}$, also in major-axis radius. The total uncertainty in the surface brightness does take into account the contribution of both local and large-scale background errors (Gil de Paz et al. 2007).

In Figure 2, we show the FUV+NUV RGB postage-stamp images. The resulting products, also shown in this figure, include the surface brightness radial profiles in both FUV and NUV in mag arcsec ${ }^{-2}$, (FUV - NUV) color profiles in mag $\operatorname{arcsec}^{-2}$, and asymptotic magnitudes (in mag) for each galaxy. The obtained values are corrected for extinction due to the Milky Way (MW). This foreground Galactic extinction was obtained following the UV extinction law of Cardelli et al. (1989), assuming a total-to-selective extinction ratio $R_{V}=A_{V} / E(B-V)=3.1$, giving the attenuation values of $A_{\mathrm{FUV}}=7.9 E(B-V)$ and $A_{\mathrm{NUV}}=8.0 E(B-V)$, where the reddening $E(B-V)$ from Galactic dust is obtained from the map of Schlegel et al. (1998). The surface photometry of the sample is not corrected for internal dust attenuation nor inclination of the host galaxy. A partial table including FUV and NUV surface photometry for 192 ETGs was first released by Zaritsky et al. (2015) and is also available in the VizieR online database (Ochsenbein et al. 2000).

In Table 3, examples of the values we obtained are shown. The graphical rendering of the data is shown in Figure 3 and is explained in the next subsection.

\subsection{IR Profile Radial Normalization}

Figure 3 shows the FUV, NUV, and $3.6 \mu \mathrm{m}$ surface brightness profiles $\mu_{\mathrm{FUV}}, \mu_{\mathrm{NUV}}$, and $\mu_{[3.6]}$ in units of mag $\operatorname{arcsec}^{-2}$ plotted against the radius in $\mathrm{kpc}$ in one case (left panels) and normalized in units of $R / R 80$ in the other (right panels).
We devised the distance unit $R / R 80$ based on the radius (i.e., semimajor axis of ellipse) that encloses $80 \%$ of the total $3.6 \mu \mathrm{m}$ light and that we call $R 80$. The innermost measurement is at $6^{\prime \prime}$ semimajor axis radius, and the rest of the measurements radially outward in the disk are represented as small dots for each $6^{\prime \prime}$ step. The very center is excluded because it could be affected by differences in the PSF among the three bands and by the contribution of an active galactic nucleus (AGN). The surface brightness (SB) measurements are taken up to $3 \times$ D25; however, for the analysis, we select only measurements having errors less than $0.2 \mathrm{mag} \mathrm{arcsec}^{-2}$. These errors include the total measurement uncertainties, dominated by Poisson noise in the centers and sky uncertainties in the outskirts, but exclude any systematic zero-point uncertainty. Color-coding is based on the numerical morphological types and is as follows: E is red, E-S0 is orange, S0 is yellow, SO-a is pink, $\mathrm{Sa}$ is light green, $\mathrm{Sb}$ is dark green, $\mathrm{Sc}$ is cyan, $\mathrm{Sd}$ is light blue, Sm is dark blue, and Irr is purple. Numerical morphological types were obtained from HyperLeda (Makarov et al. 2014) and follow the RC2 classification scheme: $-5 \leqslant \mathrm{E} \leqslant-3.5,-3.5<\mathrm{E}-\mathrm{S} 0 \leqslant-2.5, \quad-2.5<\mathrm{S} 0 \leqslant-1.5$, $-1.5<\mathrm{S} 0-\mathrm{a} \leqslant 0.5, \quad 0.5<\mathrm{Sa} \leqslant 2.5, \quad 2.5<\mathrm{Sb} \leqslant 4.5, \quad 4.5<$ $\mathrm{Sc} \leqslant 7.5, \quad 7.5<\mathrm{Sd} \leqslant 8.5, \quad 8.5<\mathrm{Sm} \leqslant 9.5, \quad$ and $\quad 9.5<$ Irr $\leqslant$ 999. Galaxies with unknown morphological types are assigned the numerical type 999 and are included in the irregular galaxies (Irr) bin, as these are, in the vast majority of cases, systems with ill-defined morphology. We list the ranges of R80 and maximum radial distances observed at $3.6 \mu \mathrm{m}$, and their respective average values per morphological type, in Table 8.

\subsection{Color Profiles}

The right column of Figure 3 shows each galaxy's spatially resolved radial color profiles in (FUV - NUV), (FUV - [3.6]), and (NUV - [3.6]) as a function of galactocentric distance both in $\mathrm{kpc}$ and in $R / R 80$ units. Each plot shows the corresponding color profile distribution for each galaxy, color-coded by morphological type. As mentioned above, measurements are taken every $6^{\prime \prime}$ from the center of each galaxy, and each profile reaches the galactocentric distance where the error in either FUV, NUV, or $3.6 \mu \mathrm{m}$ surface brightness becomes $0.2 \mathrm{mag} \operatorname{arcsec}^{-2}$ or larger, thus rejecting the data that follow. It should be noted that measurements are available up to $3 \times \mathrm{D} 25$ but are more dominated by sky uncertainties as we move radially outward.

Figure 4 shows the average surface brightnesses and colors per $R / R 80$ bin of width 0.5 , as well as the range of the scatter from the mean value in each bin and per morphological type. It should be noted that the range appears to diminish as we move radially outward, but this is due to reaching the observation limits in each band.

\section{Results}

The FUV and NUV are most sensitive to the presence and amount of (recently born) massive stars and, in particular, the FUV can be directly linked (modulo initial mass function; IMF) to the (observed) SFR, at least for late-type galaxies. In our preliminary work (Bouquin et al. 2015), we have seen that the majority of star-forming disk galaxies in our sample are distributed along the GBS; however, there exist some disk galaxies with redder, integrated (FUV - NUV) color that are located in the GGV. Spatially resolved color profiles allow us 


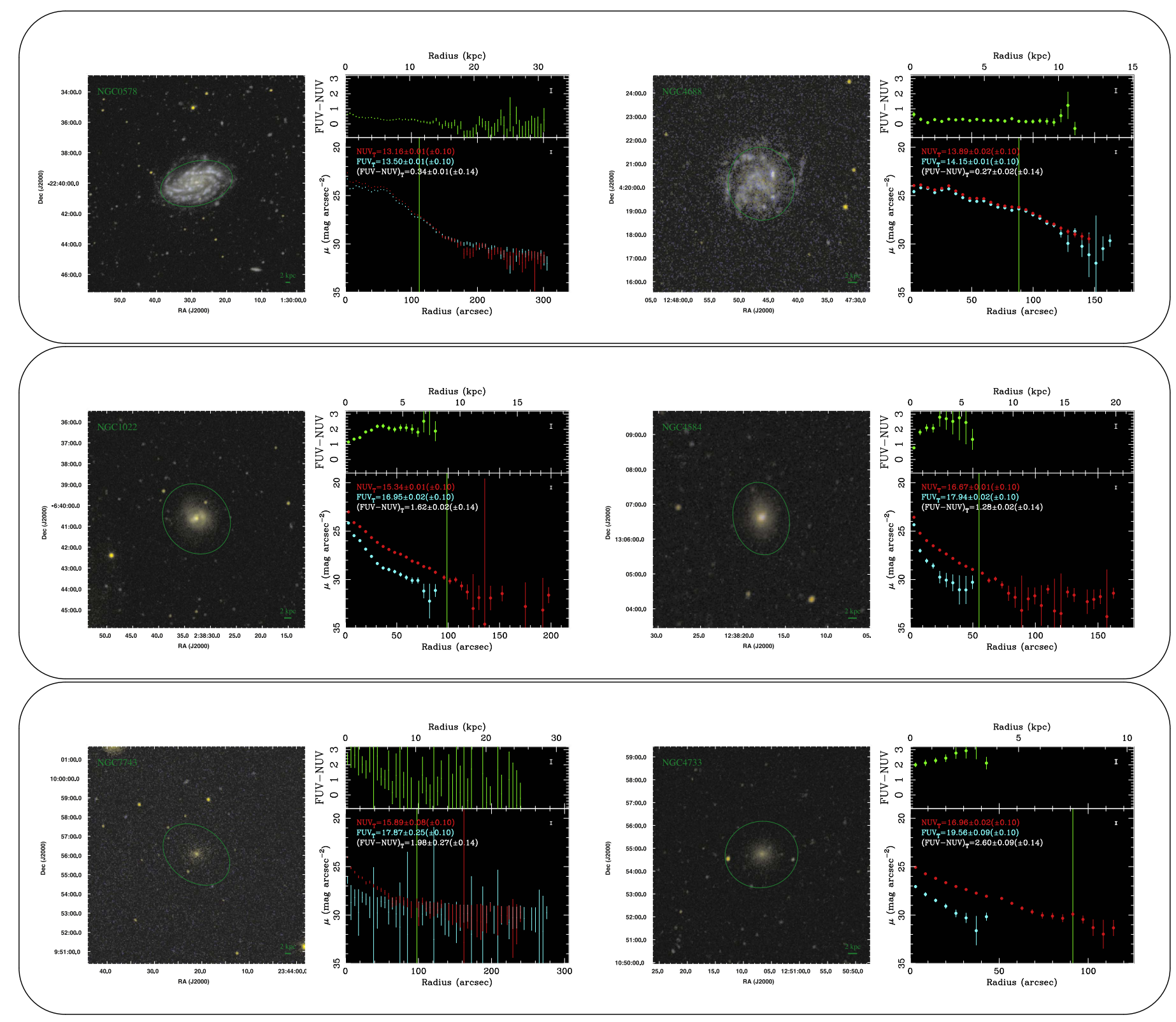

Figure 2. GALEX RGB postage-stamp images generated from FUV and NUV images (left) with their respective surface brightness $\mu_{\mathrm{FUV}}$, $\mu_{\mathrm{NUV}}$, and (FUV - NUV) color profiles (right). The first row shows typical Sc galaxies in the GBS, the second row show typical Sa galaxies in the GGV, and the third row show typical E galaxies in the GRS. The radial surface brightness profile (red dots for NUV, blue dots for FUV), as well as the (FUV - NUV) (in mag arcsec ${ }^{-2}$ ) radial color profile (green dots), are shown. The green ellipse in the RGB image corresponds to the isophotal contour D25 at $25 \mathrm{mag} \operatorname{arcsec}^{-2}$ in the $B$ band. A $2 \mathrm{kpc} s c a l e$ is shown in the bottom right corner of the image.

to see which parts of the galaxy are actually forming stars or not. Note that the (FUV - NUV) color is quite reddening-free (but not extinction-free) for MW-like foreground dust and that even if that is not the case, the effect of dust in a disk, especially in its outskirts, is smaller than that found between GBS and GRS galaxies (Muñoz-Mateos et al. 2007).

In order to study in more detail the disk component of a galaxy, we first need to isolate it by separating it from the bulge component. However, galaxies come in different shapes and sizes: some galaxies are bulgeless and only have a disk, whereas some others are diskless and only have a massive spheroidal component. We devised a method to isolate the disk component only from the $3.6 \mu \mathrm{m}$ SB profiles by applying a radial cutoff and an SB cutoff and finding the best linear fit to the outer parts of these NIR profiles (Section 4.1). This method allows us, regardless of the morphological type, to isolate the disk component and obtain its scale length and central surface brightness from the slope and $y$ intercept of the linear fit. With the spatially resolved photometry, we are able to construct a socalled star-forming main sequence, relating the FUV SB, $\mu_{\mathrm{FUV}}$, to SFR surface density and the $3.6 \mu \mathrm{m} \mathrm{SB}, \mu_{[3.6]}$, to surface stellar mass density (Section 4.2). The sSFR can be directly obtained from the (FUV - [3.6]) color (Section 4.3). We also explore the color-color diagrams obtained from these bands (Section 4.4). We show how the disks of GGV galaxies are different from those of other galaxies (Section 4.5). 
Table 3

Surface Brightness Data (Examples)

\begin{tabular}{ccccc}
\hline \hline Name & $\begin{array}{c}r \\
(\operatorname{arcsec})\end{array}$ & $\begin{array}{c}\mu_{\mathrm{FUV}} \\
\left(\mathrm{mag} \mathrm{arcsec}^{-2}\right)\end{array}$ & $\begin{array}{c}\mu_{\mathrm{NUV}} \\
\left(\mathrm{mag} \mathrm{arcsec}^{-2}\right)\end{array}$ & $\begin{array}{c}\mu_{[3.6]} \\
\left(\mathrm{mag} \mathrm{arcsec}^{-2}\right)\end{array}$ \\
\hline UGC & 6 & $26.14 \pm 0.09$ & $25.76 \pm 0.05$ & $23.16 \pm 0.03$ \\
00017 & & & & \\
& 12 & $26.32 \pm 0.10$ & $25.86 \pm 0.05$ & $23.60 \pm 0.05$ \\
& 18 & $25.96 \pm 0.05$ & $25.83 \pm 0.03$ & $24.01 \pm 0.07$ \\
& 24 & $26.47 \pm 0.05$ & $26.23 \pm 0.03$ & $24.49 \pm 0.11$ \\
& 30 & $26.65 \pm 0.05$ & $26.38 \pm 0.03$ & $24.80 \pm 0.14$ \\
& 36 & $26.91 \pm 0.06$ & $26.63 \pm 0.03$ & $24.99 \pm 0.17$ \\
& 42 & $26.66 \pm 0.05$ & $26.49 \pm 0.03$ & $25.27 \pm 0.21$ \\
& $\cdots$ & $\ldots$ & $\cdots$ & $\cdots$ \\
\hline ESO & 6 & $21.92 \pm 0.01$ & $21.89 \pm 0.01$ & $22.72 \pm 0.02$ \\
$409-$ & & & & \\
015 & & & & \\
& 12 & $23.39 \pm 0.02$ & $23.23 \pm 0.01$ & $23.50 \pm 0.04$ \\
& 18 & $24.85 \pm 0.03$ & $24.50 \pm 0.02$ & $24.24 \pm 0.07$ \\
& 24 & $25.95 \pm 0.05$ & $25.52 \pm 0.03$ & $24.98 \pm 0.14$ \\
& 30 & $26.93 \pm 0.07$ & $26.22 \pm 0.03$ & $25.08 \pm 0.15$ \\
& 36 & $27.63 \pm 0.09$ & $26.87 \pm 0.05$ & $25.62 \pm 0.24$ \\
& 42 & $28.04 \pm 0.11$ & $27.34 \pm 0.06$ & $25.85 \pm 0.29$ \\
& $\cdots$ & $\ldots$ & $\cdots$ & $\cdots$ \\
\hline
\end{tabular}

(This table is available in its entirety in machine-readable form.)

\subsection{Disk Separation Using Near-IR SB Profiles}

Disks are known to have an exponential profile and are therefore close to a straight line in a surface brightness (a logarithm) versus galactocentric radius plot, at least in their inner regions. In the very outer regions, these single exponential profiles commonly bend (see Marino et al. 2016 and references therein). It should be noted in this context, however, that the level of either downbending or upbending in the surface brightness profiles of galaxy disks is usually minimized at near-infrared wavelengths (e.g., Muñoz-Mateos et al. 2011; see also Bakos et al. 2008 for a comparison of these bending profiles at different wavelengths and in stellar mass).

In order to isolate the disk component in a coherent and reproducible way among all 1884 disk galaxies (S0 and beyond) and to derive their multiwavelength properties, we have made use of the $3.6 \mu \mathrm{m}$ surface brightness profiles of our sample and performed an error-weighted fit to our data points in $\mu_{[3.6]}$ versus galactocentric radius in kpc. Prior to this fitting, the surface brightnesses were corrected for geometrical inclination effects by adding $-2.5 \log _{10}(b / a)\left(\mathrm{mag} \operatorname{arcsec}^{-2}\right)$, where $a$ and $b$ are the semimajor and semiminor axes in the $B$ band, to each data point. No internal dust attenuation correction is applied. This has the effect of dimming the surface brightness for inclined systems (Graham \& Worley 2008). See Section 5 on how this inclination correction affects the comparison with the models. Then, we identified the position beyond which the profile starts to be best described by an exponential law at these wavelengths. In order to exclude the bulge (i.e., either the region where the Sérsic index is significantly larger than unity or the steepening associated with a pseudo-bulge), and given that we have in hand $R 80$ measurements (major-axis radius where $80 \%$ of the IR light is enclosed) for the entire sample, we remove the inner part of the profile up to some factor of $R 80$ to perform different sets of fits.
For this analysis, we explored $R / R 80$ cutoff factors of $0,0.25$, $0.50,0.75,1.00$, and 1.25 and evaluated how far we should go from the galaxy center in each case to have good linear fits, as given by the corresponding sample-averaged reduced $\chi^{2}$ values (see below). We combined this inner cutoff in $R / R 80$ with cutoffs in surface brightness magnitude in the range $\mu_{[3.6]}=21.5-24 \mathrm{mag} \mathrm{arcsec}^{-2}$, so only points fainter than the corresponding cutoff would be considered for the fit.

The rationale for using a combination of the two parameters is that we should normalize to the size of the objects to (1) do a first-order separation between bulges and disks and (2) take into account the fact that early-type systems usually have large, massive bulges with brighter near-infrared surface brightnesses than the disks of late-type spirals. Thus, when we cut in surface brightness we exclude larger regions in massive early-type systems and only the very central regions of very late-type spirals (see Figure 3, bottom right panel). However, we should certainly add a quality-of-fit criterion here to determine the goodness of these criteria.

In order to determine the reduced $\chi^{2}$ for each fit, the number of degrees of freedom is computed as the number of data points that remain after applying the corresponding cutoffs minus the number $P$ of free parameters, where $P=2$ in our linear fitting case (see Andrae et al. 2010 for a discussion). Average reduced $\chi^{2}$ values are computed for each combination of cutoffs, and the results are shown in Table 4.

When doing these fits, we excluded elliptical galaxies $(T \leqslant-3.5)$ in all cases. It should be noted that as we move toward higher values in both the $R / R 80$ and $\mu_{[3.6]}$ cutoffs, the number of points used for the linear fit decreases, and the number of galaxies that can be analyzed becomes smaller. This is because some galaxy profiles do not reach beyond the cutoffs, or only one data point is beyond them. Besides, eventually the reduced $\chi^{2}$ goes below unity, telling us that we are overfitting the data. This is in part due to the effect of correlated errors associated with the uncertainties in the sky subtraction in the very outer surface brightness measurements. We find that the best set of $R / R 80$ and $\mu_{[3.6]}$ cutoffs, i.e., the one that yields an average reduced $\chi^{2} \sim 1$ with still a large number of galaxies, is at $R / R 80=0.5$ and $\mu_{[3.6]}=23.5$ mag $\operatorname{arcsec}^{-2}$, where $\left\langle\chi^{2}\right\rangle=1.12$ and the number of galaxies is 987 ( $\sim 51 \%$ of the $G A L E X / \mathrm{S}^{4} \mathrm{G}$ sample; see Figure 5).

We also apply oblique cuts in the $\mu_{[3.6]}$ versus $R / R 80$ plane instead of a combination of vertical and horizontal cuts. Table 5 shows the resulting average reduced $\chi^{2}$ and the number of galaxies for a combination of cutoff slopes $a$ and cutoff $y$ intercepts $b$. We tried all the combinations of slopes ranging from -7 to -1 (in units of mag $\operatorname{arcsec}^{-2} /(R / R 80)$ ) and $y$ intercepts between 20 and $30 \mathrm{mag} \mathrm{arcsec}{ }^{-2}$. The best compromise between the average reduced $\chi^{2}$ and number of galaxies is for slope and $y$-intercept values of $a=-1$ and $b=24 \mathrm{mag} \mathrm{arcsec}^{-2}$, where the average reduced $\chi^{2}=2.07$ and the number of galaxies is 1233 ( $\sim 64 \%$ of the GALEX $/ \mathrm{S}^{4} \mathrm{G}$ sample; see Figure 5). Graphical representations of the slopes and yintercepts at these best cutoffs are shown in Figure 6.

The relatively good isolation of the disk component by some of these sets of criteria opens the door to statistical studies of the photometric properties of disks in thousands or millions of galaxies using existing data (SDSS) or data from future facilities and missions, such as the Large Synoptic Survey Telescope (LSST) or EUCLID. 

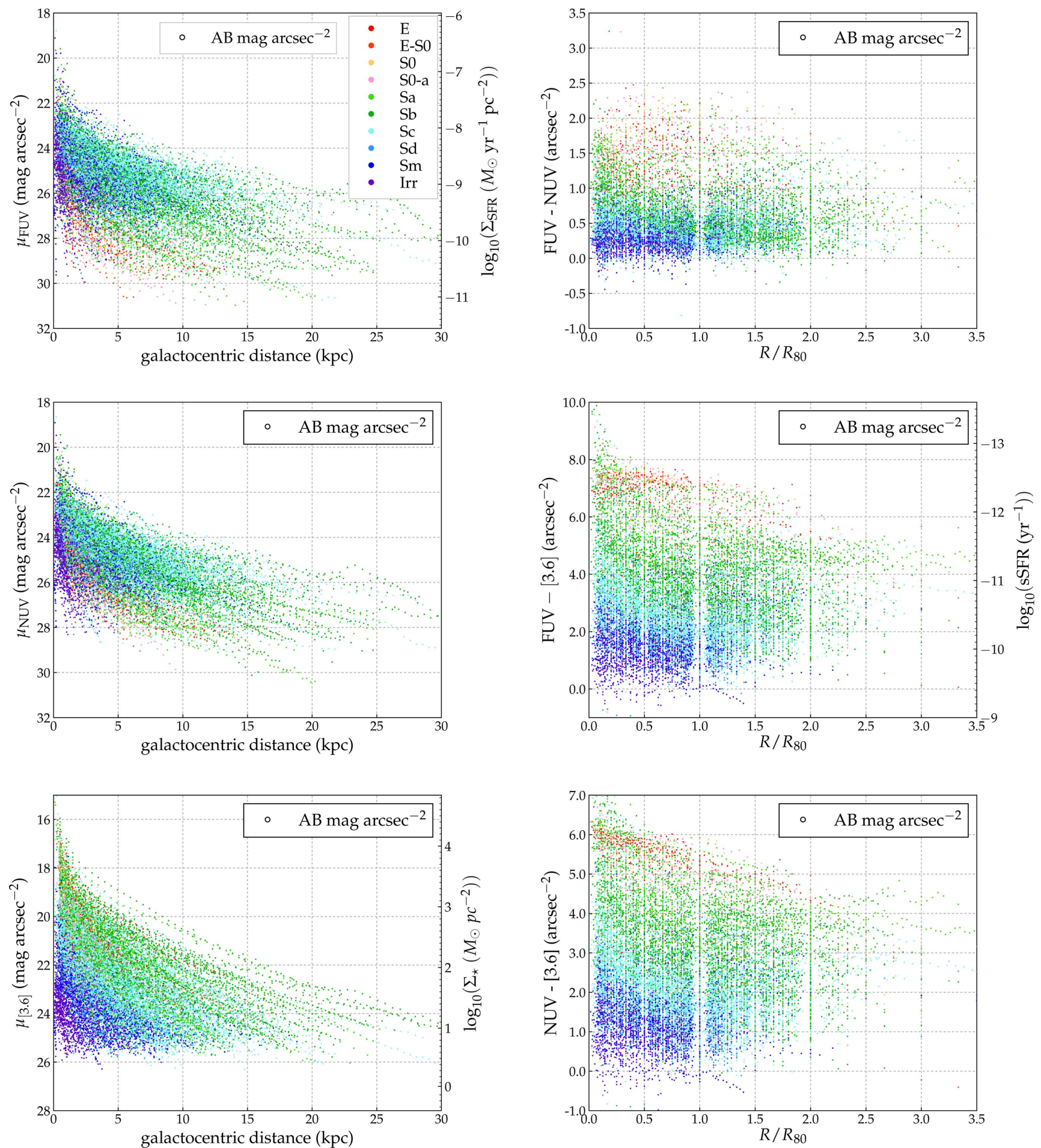

Figure 3. Left column, top to bottom: FUV, NUV, and [3.6] surface brightness vs. radius in kpc. Right column, top to bottom: (FUV - NUV), (FUV - [3.6]), and (NUV - [3.6]) colors vs. $R / R 80$. Each dot represents a data point. Our entire sample of 1931 galaxies is shown. Color-coding is based on the numerical morphological types: E is red, E-S0 is orange, S0 is yellow, S0-a is pink, Sa is light green, Sb is dark green, Sc is cyan, Sd is light blue, Sm is dark blue, and Irr is purple. The discretization seen in the right-hand plots is due to the fact that the $R 80$ values derived from the analysis of our growth curves are obtained from the data point that encompasses a fraction of the light closest to $80 \%$, but it is not interpolated. The figure shows that this translates into an error of no more than $\pm 0.1 R / R 80$.

\subsection{Spatially Resolved Star-forming Main Sequence from UV and Near-IR SB Profiles}

In Figure 7 , we plot the FUV surface brightness $\mu_{\mathrm{FUV}}$ versus the $3.6 \mu \mathrm{m}$ surface brightness $\mu_{[3.6]}$ for galaxies belonging to the GBS, GGV, and GRS subsamples based on their integrated colors. Both axes are expressed in mag $\operatorname{arcsec}^{-2}$.

This figure can also be seen as a comparison between the observed SFR (i.e., not corrected for internal dust extinction) and the stellar mass surface densities (see Appendix A), except 

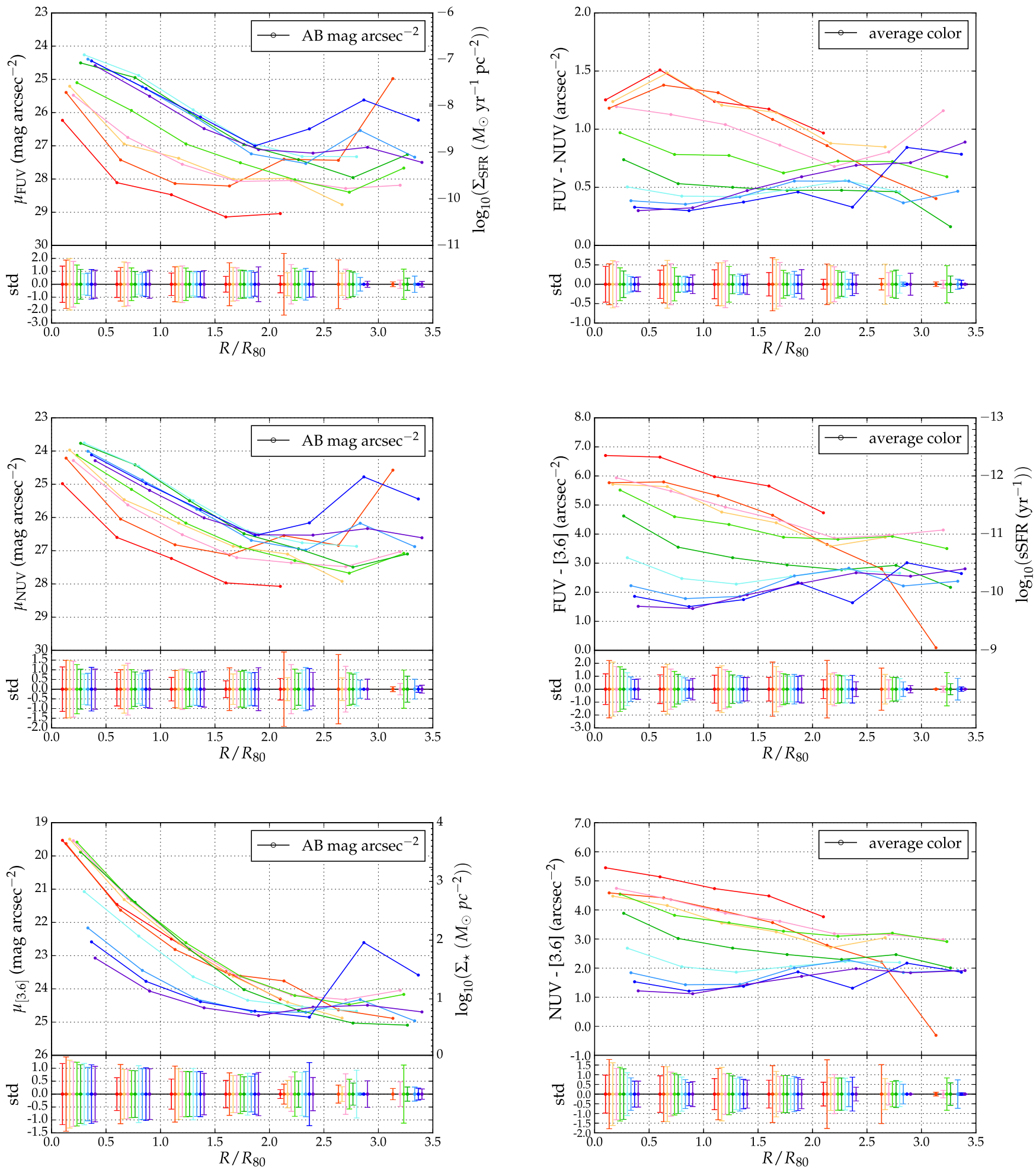

Figure 4. Top left: average surface brightness color-coded per morphological type per $R / R 80$ bin of width 0.5 . Bottom left: standard deviation (std) of the scatter from the mean, including the uncertainty, within each bin. A translation in $x$ is applied for better visibility. It should be noted that the sample size substantially drops beyond $R / R 80>1.5$ due to the observation limits in each band. Right column: Same as the left column but for color profiles.

for those cases where the FUV emission is not due to young massive stars. In that regard, this point is equivalent to the star formation main sequence (SFMS) but in surface brightness (see Cano-Díaz et al. 2016).

Each data point is the averaged value within fixed-inclination elliptical ring apertures of $6 "$ width. The innermost ring has a semimajor axis length of $6^{\prime \prime}$ with a width of $6^{\prime \prime}$, defined by an inner ellipse with a major axis of $3^{\prime \prime}$ from the center and an outer ellipse with a semimajor axis of $9^{\prime \prime}$ from the center. The initial ring does not cover the center of the galaxy, as this could be affected by differences in the PSF among the three bands and by the contribution of an AGN. Subsequent rings increase 


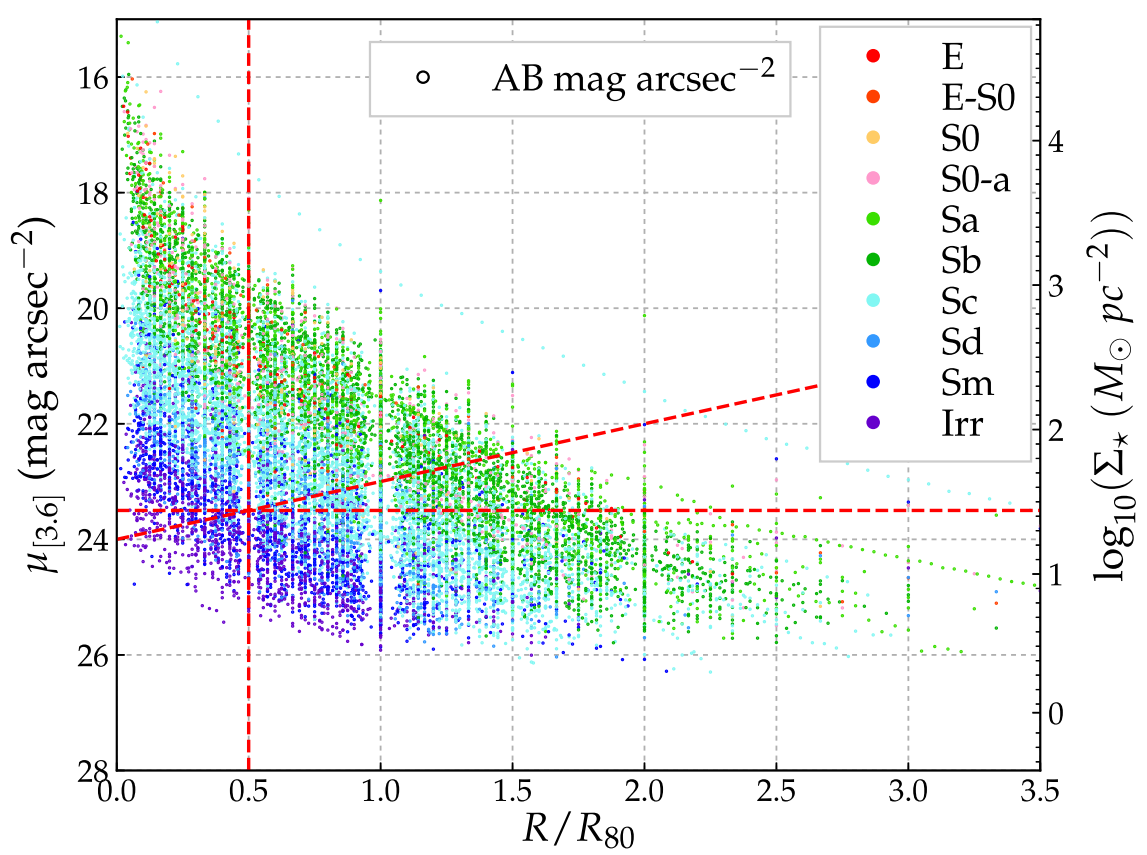

Figure 5. The $3.6 \mu \mathrm{m}$ surface brightness profiles normalized to $R 80$. The red dashed lines represent one example of the vertical and horizontal cutoffs and another example of an oblique cutoff.

Table 4

Average Reduced $\chi^{2}$ of the Linear Fit with $\mu_{[3.6]}$ and $R / R 80$ Cuts

\begin{tabular}{|c|c|c|c|c|c|c|c|c|c|c|c|c|c|}
\hline \multicolumn{14}{|c|}{$R / R 80$ Cutoffs } \\
\hline & & \multicolumn{2}{|c|}{0.00} & \multicolumn{2}{|c|}{0.25} & \multicolumn{2}{|c|}{0.50} & \multicolumn{2}{|c|}{0.75} & \multicolumn{2}{|c|}{1.00} & \multicolumn{2}{|c|}{1.25} \\
\hline & & $\left\langle\chi^{2}\right\rangle$ & $N^{\mathrm{a}}$ & $\left\langle\chi^{2}\right\rangle$ & $N$ & $\left\langle\chi^{2}\right\rangle$ & $N$ & $\left\langle\chi^{2}\right\rangle$ & $N$ & $\left\langle\chi^{2}\right\rangle$ & $N$ & $\left\langle\chi^{2}\right\rangle$ & $N$ \\
\hline \multirow[t]{6}{*}{$\mu_{[3.6]}$ cutoffs } & 21.5 & 26.20 & (1577) & 20.84 & (1554) & 15.72 & $(1451)$ & 9.68 & (1240) & 4.04 & (794) & 2.87 & $\overline{(535)}$ \\
\hline & 22 & 10.64 & (1489) & 8.63 & (1474) & 6.97 & (1387) & 5.85 & (1191) & 3.26 & (781) & 2.48 & (530) \\
\hline & 22.5 & 4.89 & (1384) & 4.28 & (1375) & 3.54 & (1298) & 3.11 & (1126) & 2.02 & (756) & 1.62 & (518) \\
\hline & 23 & 2.34 & (1232) & 2.17 & (1228) & 1.81 & (1165) & 1.63 & (1014) & 1.14 & (693) & 0.98 & (482) \\
\hline & 23.5 & 1.37 & (1034) & 1.28 & (1033) & 1.12 & $(987)$ & 0.96 & (863) & 0.68 & (591) & 0.56 & (419) \\
\hline & 24 & 0.78 & $(755)$ & 0.77 & (754) & 0.73 & (723) & 0.67 & (630) & 0.40 & (426) & 0.35 & (296) \\
\hline
\end{tabular}

Note.

${ }^{a} N$ is the number of galaxies remaining after applying the cutoffs and on which the linear fitting is performed.

in size in $6^{\prime \prime}$ steps, i.e., they have semimajor axis radii of $12^{\prime \prime}$, $18^{\prime \prime}, 24^{\prime \prime}$, and so on.

For early-type GRS galaxies, the FUV and $3.6 \mu \mathrm{m}$ surface brightnesses show a pretty tight correlation, which indicates that the $3.6 \mu \mathrm{m}$ emission traces not only the stellar mass but also the bulk of the stars dominating the FUV emission in these objects, mainly main-sequence turnoff or extreme horizontal branch (EHB) stars, depending on the strength of the UV upturn. Despite the large scatter of the GRS found in Bouquin et al. (2015), the use of spatially resolved data with the $3.6 \mu \mathrm{m}$ surface brightness as normalizing parameter leads now to a very tight GRS in this SB-SB plane (or a very small range in FUV - [3.6] color). The comparison of these profiles with those of the GGV galaxies shows that in the latter case the central stellar mass surface density is 1.5-2 mag fainter than in the former and that most GGV galaxies (all except the few very late-type GGVs) have (outer) disks that follow a trend similar to that followed by the outer regions of GRS galaxies. Finally, late-type galaxies in the GBS span a large range of values in both $\mu_{\mathrm{FUV}}$ and $\mu_{[3.6]}$. Irregular, Sm, and Sd galaxies have the highest SFR surface densities (for a given stellar mass surface density) among the GBS subsample.

Despite the large scatter of GBS galaxies, they can be clearly distinguished from the ETGs of the GRS and even GGV galaxies by looking at the (observed) sSFR values in their disks. Thus, while GBS disks have sSFR values that are higher than $10^{-11.5} \mathrm{yr}^{-1}$, the outer regions of GGV and GRS galaxies are in the majority of cases (all in the case of the GRS) below this value. This value could be used to easily discriminate between star-forming and quiescent regions within galaxies.

GBS galaxies define a well-separated sequence, and with the spatial information now available, we can see what parts of the galaxies are now just leaving the GBS, that is, have their star formation suppressed or exhausted. While a few GGV galaxies show a decrease in the sSFR of their inner regions, most of these galaxies are within the locus of the GBS in the inner parts but approach the sequence marked by the GRS profiles in their outer regions. In other words, the fact that these galaxies were identified as leaving the GBS in Bouquin et al. (2015) is mainly due to their outer parts, likely caused by the disks of GGV 

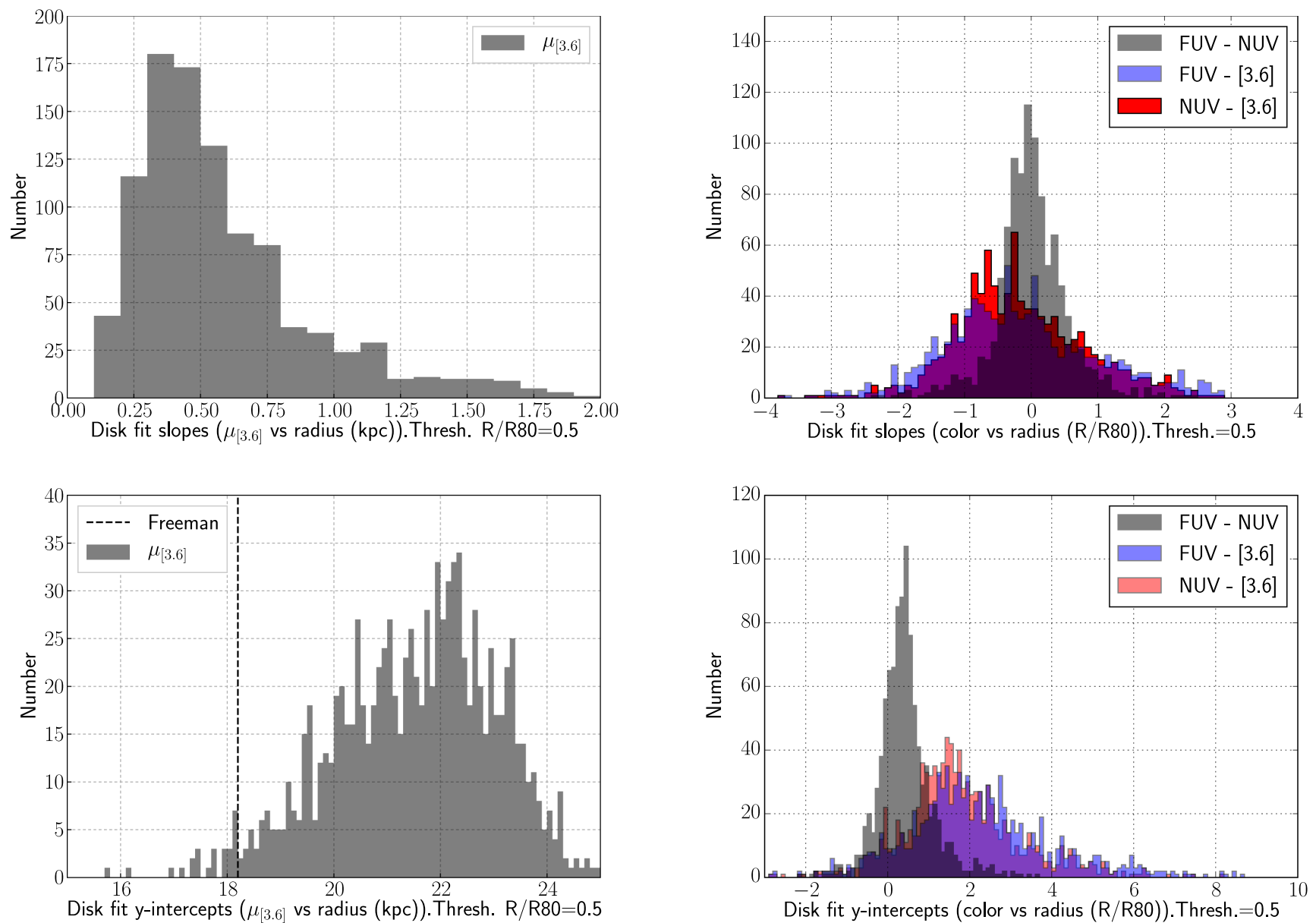

Figure 6. Distributions of the best-fitting coefficients to the surface brightness and color profiles of disks. The fitting is performed beyond a radius $R / R 80=0.5$ using points where the surface brightness is fainter than $\mu_{[3.6]}=23.5$ mag arcsec ${ }^{-2}$. Top left: distribution of slopes obtained in the $\mu_{[3.6]} \mathrm{vs}$. kpc plane. Bottom left: distribution of $y$ intercepts obtained in that plane. These correspond to the central surface brightness contribution of the disks. The dashed vertical line corresponds to the Freeman (1970) value, or the $B$-band central surface brightness for spirals $\left\langle\Sigma_{0}\right\rangle=21.48 B$-mag $\operatorname{arcsec}^{-2}$, converted to a $3.6 \mu \mathrm{m}$ value of $18.2 \mathrm{mag}$ arcsec ${ }^{-2}$, assuming an average central color of $\left(B_{\mathrm{Vega}}-[3.6]_{\mathrm{AB}}\right)=3.32 \mathrm{mag}$. Top right: distribution of slopes obtained in the (FUV $\left.-\mathrm{NUV}\right)$, (FUV $\left.-[3.6]\right)$, and (NUV - [3.6]) vs. $R / R 80$ planes, i.e., the color gradients. Bottom right: distribution of $y$ intercepts obtained in those planes, or central colors of the disks. Bin width is 0.1 in all cases (in units of either mag or $\mathrm{mag} /(R / R 80)$ ).

Table 5

Average Reduced $\chi^{2}$ of the Linear Fit in the $\mu_{[3.6]}$ vs. $R / R 80$ Plane with Oblique Cuts

\begin{tabular}{|c|c|c|c|c|c|c|c|c|c|c|c|c|c|}
\hline \multicolumn{14}{|c|}{ Slope (a) Cutoff } \\
\hline & & \multicolumn{2}{|c|}{-6} & \multicolumn{2}{|c|}{-5} & \multicolumn{2}{|c|}{-4} & \multicolumn{2}{|c|}{-3} & \multicolumn{2}{|c|}{-2} & \multicolumn{2}{|c|}{-1} \\
\hline & & $\left\langle\chi^{2}\right\rangle$ & $N$ & $\left\langle\chi^{2}\right\rangle$ & $N$ & $\left\langle\chi^{2}\right\rangle$ & $N$ & $\left\langle\chi^{2}\right\rangle$ & $N$ & $\left\langle\chi^{2}\right\rangle$ & $N$ & $\left\langle\chi^{2}\right\rangle$ & $N$ \\
\hline & 22 & 205.61 & (1697) & 158.46 & (1691) & 129.09 & (1684) & 88.95 & (1668) & 52.78 & (1644) & 27.10 & (1592) \\
\hline & 24 & 61.96 & (1633) & 44.50 & (1607) & 28.48 & (1563) & 13.20 & (1528) & 5.58 & (1412) & 2.07 & (1233) \\
\hline & 25 & 33.19 & (1578) & 23.64 & (1540) & 11.19 & (1482) & 6.19 & (1375) & 2.21 & (1202) & 0.78 & (831) \\
\hline & 30 & 2.46 & (858) & 1.56 & (596) & 0.87 & (322) & 1.27 & (103) & 1.04 & (8) & $\ldots$ & (0) \\
\hline
\end{tabular}

galaxies undergoing either an outside-in star formation quenching or an inside-out rebirth.

It is worth emphasizing here that only the combined use of FUV, NUV, and $3.6 \mu \mathrm{m}$ allows for properly separating the "classical blue cloud" (now blue sequence) and "classical red sequence" and determining which galaxies are now leaving (or entering) the GBS and what regions within the galaxies are responsible for it.
We mark in Figure 7 the $\mu_{[3.6]}$ value that corresponds to the surface stellar mass density of $\Sigma_{\star}=3 \times 10^{8} M_{\odot} \mathrm{kpc}^{-2}=$ $300 M_{\odot} \mathrm{pc}^{-2}\left(\mu_{[3.6]}=20.89 \mathrm{mag} \operatorname{arcsec}^{-2}\right)$ proposed by Kauffmann et al. (2006) to separate the bulge-dominated and disk-dominated objects.

In the case of our GBS galaxies, this stellar mass surface density indicates the region inside which the SFR surface density flattens relative to the stellar mass surface density, i.e., 

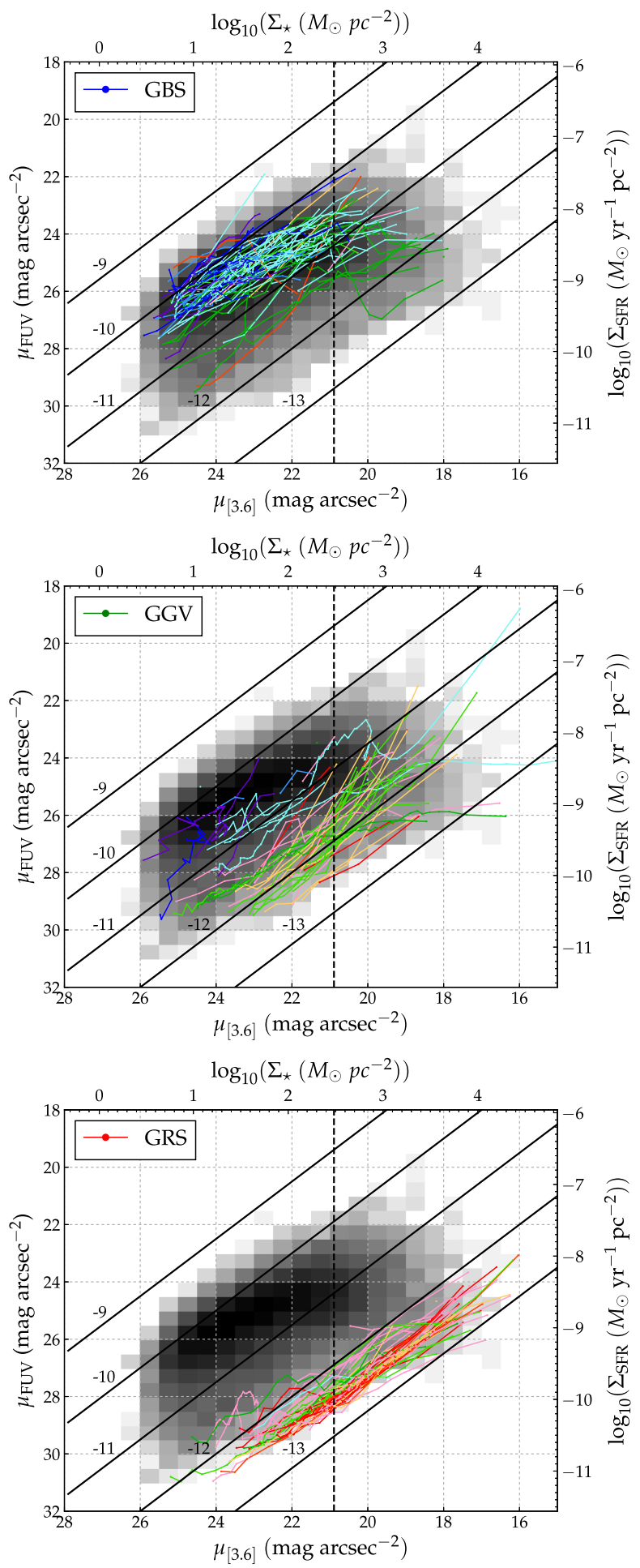

Figure 7. FUV surface brightness vs. $3.6 \mu \mathrm{m}$ surface brightness for randomly selected GBS (top), all GGV (middle), and all GRS (bottom) galaxy subsamples. The diagonal solid lines represent constant sSFRs and are annotated with the decimal exponent of the logarithm. These plots are equivalent to the (observed) SFMS but in surface brightness. Both the segregation in sSFR between the GBS, GGV, and GRS and the bending at high (surface density) masses toward lower sSFR values are also clear in this plot. The vertical black dashed line corresponds to $\Sigma_{\star}=3 \times 10^{8} \quad M_{\odot} \quad \mathrm{kpc}^{-2}$ (or $\mu_{[3.6]}=20.89 \mathrm{mag} \operatorname{arcsec}^{-2}$; Kauffmann et al. 2006). The $2 \mathrm{D}$ density histogram shown in the background of each panel represents the data-point density of GBS galaxies.

when the (FUV - [3.6]) color becomes significantly redder (see Figure 9). A similar change is observed when using the light-

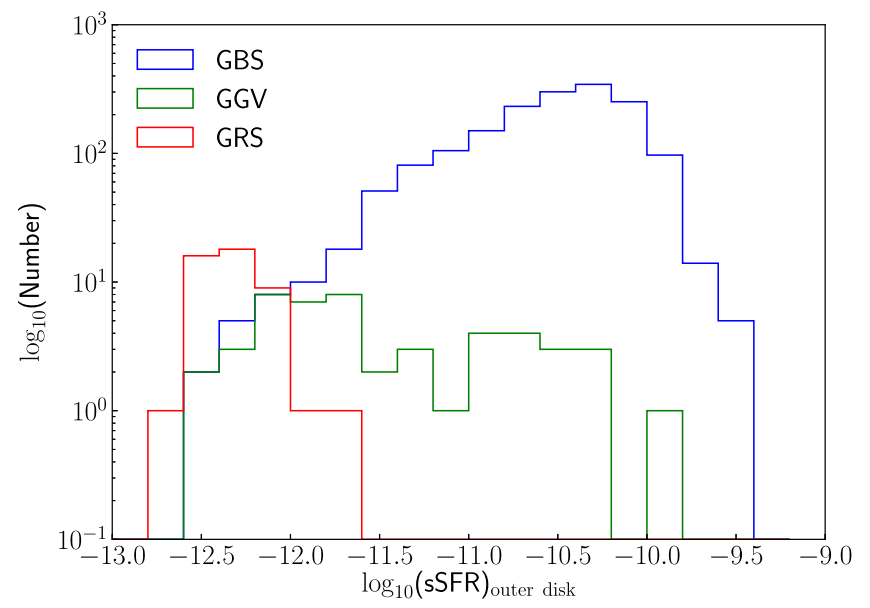

Figure 8. Decimal log-log histogram of the mean sSFR obtained by fitting the outer disk part (beyond $\mu_{[3.6]}=20.89 \mathrm{mag} \mathrm{arcsec}^{-2}$ ) of GBS, GGV, and GRS galaxies. The bin size is 0.2 dex.

weighted age of the stellar population in galaxies instead (González Delgado et al. 2014).

The sSFR of the outer parts (beyond $\mu_{[3.6]}=20.89$ mag $\operatorname{arcsec}^{-2}$ ) is shown in Figure 8 for GBS, GGV, and GRS galaxies. This is done simply by calculating the linear scale sSFR of one galaxy at each point that is in the outer parts and averaging these sSFR values (not light/mass-weighted) in order to get a single sSFR value per galaxy (and expressing them in the logarithmic scale at the end). We find the following sSFR density ranges: $-12.5<\log _{10}(\mathrm{sSFR})<-9.5$ for GBS galaxies, $-12.4<\log _{10}(\mathrm{sSFR})<-9.8$ for GGV galaxies, and $-12.6<$ $\log _{10}(\mathrm{SSFR})<-11.7$ for GRS galaxies. Since we do not correct for internal dust attenuation, these values should be viewed as lower limits of the true sSFR. Previous studies of the impact of dust on the (FUV - [3.6]) colors (Muñoz-Mateos et al. 2007, 2009a, 2009b) have shown that dust attenuation $A_{\mathrm{FUV}}$ decreases as we move outward in the disks, although the dust content differs from one morphological type bin to another; for example, $\mathrm{Sb}-\mathrm{Sbc}$ galaxies have higher $A_{\mathrm{FUV}}$ at all radii than the other types, whereas Sdm-Irr have relatively very low dust content. It should be noted, however, that besides dust, the reddening in the outer parts of quiescent galaxies is due to their older stars. There is a clear difference between the outer parts of GRS galaxies having low sSFR and a narrow range of values and those of GBS galaxies with a wide range of sSFR but in general not as low as the outer parts of the GRS. For our sample, we have a distribution in outer disk sSFR with the mean at -10.6 and $\sigma=0.5 \mathrm{dex}$ (rms) for GBS, -11.5 and $\sigma=0.7$ dex for GGV, and -12.3 and $\sigma=0.2$ dex for GRS galaxies. The sSFR of the outer parts of the GGV galaxies in our sample covers a wider range of values but is not as high as that of some GBS galaxies and not as low as that of some GRS galaxies. Note that in the case of the GRS galaxies, the UV emission might not be due to recent star formation but to the light from low-mass evolved stars.

\subsection{Color and sSFR Profiles}

Figure 9 shows the GRS (top), GGV (middle), and GBS (bottom) galaxies' (FUV - [3.6]) color profiles versus $3.6 \mu \mathrm{m}$ surface brightness $\mu_{[3.6]}$, with the same color-coding per morphological type as in previous plots. Again, the $3.6 \mu \mathrm{m}$ surface brightness corresponds to the stellar mass per area (see 

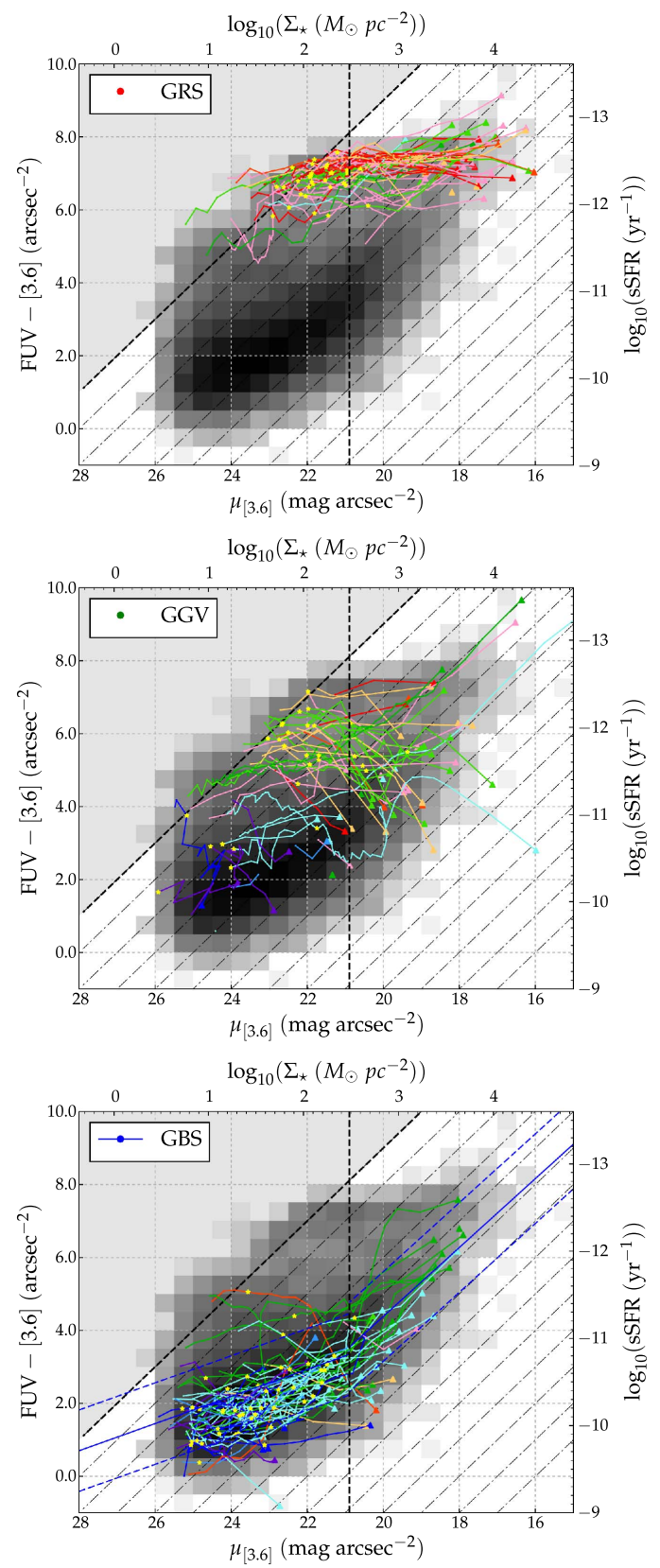

Figure 9. The (FUV - [3.6]) color vs. $\mu_{[3.6]}$ surface brightness for the radial profiles of GRS (top), GGV (middle), and GBS (bottom) galaxies. Each galaxy's center (within $6^{\prime \prime}$ of the central-most aperture) is represented by a triangle, and subsequent values are taken every $6^{\prime \prime}$ and represented by smaller dots if these values exist. Values (dots) belonging to the same galaxy are connected by a line of the same color as the dots. The yellow star shows the radial distance at which $80 \%$ of the $3.6 \mu \mathrm{m}$ light is enclosed. Diagonal dot-dashed lines are lines of constant $\mu_{\mathrm{FUV}}$ $\operatorname{arcsec}^{-2}$ (i.e., observed SFR surface density), with the left-most dashed line corresponding to $\mu_{\mathrm{FUV}}=29 \mathrm{AB}$ mag $\operatorname{arcsec}^{-2}$ (corresponding to $\Sigma_{\mathrm{SFR}}=$ 4. $36 \times 10^{-5} M_{\odot} \mathrm{yr}^{-1} \mathrm{kpc}^{-2}$ for a Kroupa IMF), the approximate sensitivity limit of our GALEX observations. The vertical black dashed line corresponds to $\Sigma_{\star}=3 \times 10^{8} M_{\odot} \mathrm{pc}^{-2}\left(\right.$ or $\mu_{[3.6]}=20.89 \mathrm{mag} \operatorname{arcsec}^{-2}$; Kauffmann et al. 2006). The solid blue lines that go through the data points in the GBS plot are the fits to all the data points on each side of $\mu_{[3.6]}=20.89$. The parallel dashed blue lines show the $\pm 1 \sigma$ (rms) of the distribution. In the case of the GBS plot, we show randomly selected galaxies to better illustrate how GBS galaxies behave. In all cases, the entirety of the data is shown as a logarithmic $2 \mathrm{D}$ density histogram with $0.5 \times 0.5$ binning, where darker (brighter) shades mean higher (lower) data-point density.

Equation (14) in Appendix A), and the (FUV - [3.6]) color is equivalent to the observed (not corrected for internal extinction) sSFR (units $\mathrm{yr}^{-1}$; see Equation (20) in Appendix B).
The yellow star symbol corresponds to the radial measurement where the cumulative magnitude at $3.6 \mu \mathrm{m}$ reaches $80 \%$ of the enclosed light at this wavelength.

The (FUV - [3.6]) color profiles are very different for the GRS, GGV, and GBS subsamples. In the case of GRS galaxies, which are mostly early-type but not exclusively, the color is tightly constrained within a range from 6 to 8 mag but gets a bit bluer to the outer regions, especially for GRS galaxies of S0, $\mathrm{Sa}, \mathrm{Sb}$, and $\mathrm{Sc}$ morphological types.

On the other hand, in the case of GBS galaxies, their (FUV - [3.6]) color ranges from -1 to $10 \mathrm{mag}$, corresponding to an sSFR value ranging from $10^{-10}$ to $10^{-13} \mathrm{yr}^{-1}$. Regarding the differences in the color profiles for each galaxy type, Sa, $\mathrm{Sb}$, and $\mathrm{Sc}$ galaxies go from red to blue from the inside out, while $\mathrm{Sd}, \mathrm{Sm}$, and Irregulars are much bluer than $\mathrm{Sa}, \mathrm{Sb}$, and Sc at a given stellar mass surface density but their color gradients are somewhat flatter. Again, it should be noted that we are not correcting for dust and that the effect of dust is to redden the (FUV - [3.6]) color (Muñoz-Mateos et al. 2007) and therefore yields a lower limit to the sSFR.

The fact that most profiles of GBS galaxies become bluer from the inside out indicates that the lower the surface stellar mass density (the greater the galactocentric distance), the greater the sSFR, i.e., the higher the SFR for a given surface stellar mass density, the more stars are born in the outskirts. Correcting for internal dust extinction, assuming that dust extinction and reddening effects are stronger in the inner regions than in the outer parts, would yield bluer centers compared to the outer disk. This has the effect of increasing the slope of the gradient, where negative color gradients would become flatter and positive color gradients even more positive. Such an effect would translate to a less-pronounced degree of inside-out growth. It should be noted that while the internal dust correction would affect the color profiles of the galaxies, it is not enough to explain why most galaxies are becoming bluer from the inside out (see Figure 2 in Muñoz-Mateos et al. 2007). Studies by Muñoz-Mateos et al. $(2007,2011)$ and Pezzulli et al. (2015) on nearby galaxy samples have shown that mass and radial growth of nearby spiral disks growing from the inside out have timescales on the order of $\sim 10$ and $30 \mathrm{Gyr}$, respectively. Isolating a few galaxies actively forming stars in their outer regions reveals that their outer regions indeed fall near $\log _{10}(\mathrm{sSFR}) \sim-10 \mathrm{yr}^{-1}$, or $\sim 10 \mathrm{Gyr}$, in agreement with the above work (see GBS plot in Figure 9). In the case of profiles reddening in the outskirts, the lower $\Sigma_{\star}$ becomes, the smaller the sSFR.

Remarkably, a clear color flattening is observed in the outer parts of the profiles of most GBS galaxies when $\Sigma_{\star}<300 M_{\odot}$ $\mathrm{pc}^{-2}$. Applying a weighted linear fit to the left-hand and right-hand sides of $\mu_{[3.6]}=20.89 \mathrm{mag} \operatorname{arcsec}^{-2}$, we get $($ FUV $-[3.6])=(-0.395 \pm 0.023) \cdot \mu_{[3.6]}+(11.537 \pm 0.453)$ and $(-0.438 \pm 0.009) \cdot \mu_{[3.6]}+(12.210 \pm 0.193)$, respectively. These are shown in Figure 9 as solid blue lines for the mean value accompanied by parallel dashed blue lines corresponding to the $1 \sigma$ uncertainty.

The galaxies falling into the GGV category globally are also clearly distinct from the GBS ones in terms of their spatially resolved properties. They show flat or even inverted color (and sSFR) profiles as a function of stellar mass surface density (hardly due to radial variations in the amount of dust reddening; see Muñoz-Mateos et al. 2007), which indicates either a decline in the observed SFR (oblique lines in Figure 9) 
Table 6

Color and Surface Brightness Gradients from Radial Profiles Normalized to $R 80$ (Except for $\mu_{[3.6]}$ )

\begin{tabular}{|c|c|c|c|c|c|c|c|c|}
\hline \multirow{3}{*}{$\begin{array}{l}\text { Unit } \\
\text { Name }^{a}\end{array}$} & \multicolumn{2}{|c|}{ FUV - NUV } & \multicolumn{2}{|c|}{ FUV - [3.6] } & \multicolumn{2}{|c|}{$\mathrm{NUV}-[3.6]$} & \multicolumn{2}{|c|}{$\mu_{[3.6]}$} \\
\hline & \multicolumn{2}{|c|}{$\mathrm{mag} /(R / R 80)$} & \multicolumn{2}{|c|}{$\mathrm{mag} /(R / R 80)$} & \multicolumn{2}{|c|}{$\mathrm{mag} /(R / R 80)$} & \multicolumn{2}{|c|}{ mag kpc ${ }^{-1}$} \\
\hline & $a^{\mathrm{b}}$ & $b^{\mathrm{c}}$ & $\bar{a}$ & $\bar{b}$ & $\bar{a}$ & $\bar{b}$ & $\bar{a}$ & $\bar{b}$ \\
\hline ESO 293-034 & $-0.03 \pm 0.06$ & $0.48 \pm 0.07$ & $-1.31 \pm 0.12$ & $4.30 \pm 0.13$ & $-1.37 \pm 0.09$ & $3.91 \pm 0.09$ & $0.55 \pm 0.02$ & $21.41 \pm 0.14$ \\
\hline NGC 0007 & $0.15 \pm 0.42$ & $0.21 \pm 0.34$ & $-0.11 \pm 0.48$ & $1.64 \pm 0.39$ & $-0.25 \pm 0.14$ & $1.42 \pm 0.11$ & $\ldots \pm \ldots$ & $\ldots \pm \ldots$ \\
\hline IC 1532 & $-0.76 \pm 0.92$ & $0.96 \pm 0.66$ & $-0.12 \pm 0.95$ & $2.44 \pm 0.67$ & $0.63 \pm 0.01$ & $1.48 \pm 0.01$ & $\ldots \pm \ldots$ & $\ldots \pm \ldots$ \\
\hline NGC 0024 & $0.00 \pm 0.02$ & $0.34 \pm 0.02$ & $-0.54 \pm 0.05$ & $3.04 \pm 0.04$ & $-0.58 \pm 0.05$ & $2.74 \pm 0.04$ & $1.04 \pm 0.03$ & $20.81 \pm 0.12$ \\
\hline ESO 293-045 & $0.05 \pm 0.07$ & $0.08 \pm 0.06$ & $-0.83 \pm 0.41$ & $1.20 \pm 0.33$ & $-0.92 \pm 0.36$ & $1.15 \pm 0.29$ & $0.64 \pm 0.03$ & $23.15 \pm 0.12$ \\
\hline UGC 00122 & $1.01 \pm 0.21$ & $-0.63 \pm 0.16$ & $1.16 \pm 0.30$ & $-0.23 \pm 0.23$ & $0.15 \pm 0.25$ & $0.40 \pm 0.19$ & $0.81 \pm 0.04$ & $24.24 \pm 0.10$ \\
\hline NGC 0059 & $0.09 \pm 0.11$ & $1.60 \pm 0.09$ & $0.18 \pm 0.11$ & $4.82 \pm 0.09$ & $0.10 \pm 0.05$ & $3.21 \pm 0.04$ & $2.23 \pm 0.07$ & $21.38 \pm 0.09$ \\
\hline ESO 539-007 & $-1.11 \pm 1.14$ & $0.97 \pm 0.90$ & $-5.36 \pm 0.44$ & $4.58 \pm 0.33$ & $-3.73 \pm 0.71$ & $3.22 \pm 0.50$ & $0.24 \pm 0.04$ & $24.37 \pm 0.14$ \\
\hline ESO 150-005 & $-0.27 \pm 0.18$ & $0.36 \pm 0.14$ & $-0.77 \pm 0.50$ & $1.89 \pm 0.35$ & $-0.56 \pm 0.40$ & $1.57 \pm 0.28$ & $0.24 \pm 0.04$ & $24.19 \pm 0.14$ \\
\hline NGC 0100 & $0.87 \pm 0.18$ & $0.02 \pm 0.12$ & $-1.15 \pm 0.85$ & $4.10 \pm 0.57$ & $-2.05 \pm 0.68$ & $4.10 \pm 0.45$ & $\ldots \pm \ldots$ & $\ldots \pm \ldots$ \\
\hline NGC 0115 & $0.06 \pm 0.04$ & $0.16 \pm 0.04$ & $-0.48 \pm 0.13$ & $1.83 \pm 0.11$ & $-0.51 \pm 0.13$ & $1.63 \pm 0.11$ & $0.52 \pm 0.03$ & $21.27 \pm 0.17$ \\
\hline UGC 00260 & $0.04 \pm 0.07$ & $0.29 \pm 0.09$ & $-1.29 \pm 0.13$ & $3.76 \pm 0.16$ & $-1.43 \pm 0.14$ & $3.57 \pm 0.16$ & $0.27 \pm 0.02$ & $22.96 \pm 0.26$ \\
\hline NGC 0131 & $0.11 \pm 0.08$ & $0.34 \pm 0.08$ & $0.00 \pm 0.12$ & $2.86 \pm 0.10$ & $-0.15 \pm 0.08$ & $2.55 \pm 0.06$ & $\ldots \pm \ldots$ & $\ldots \pm \ldots$ \\
\hline UGC 00320 & $0.12 \pm 0.17$ & $0.20 \pm 0.15$ & $0.15 \pm 0.46$ & $1.61 \pm 0.36$ & $-0.01 \pm 0.31$ & $1.44 \pm 0.23$ & $0.50 \pm 0.03$ & $22.88 \pm 0.17$ \\
\hline $\operatorname{Total}^{\mathrm{d}}$ & 1541 & & 1541 & & 1541 & & 992 & \\
\hline
\end{tabular}

Notes.

a Same nomenclature as the $\mathrm{S}^{4} \mathrm{G}$.

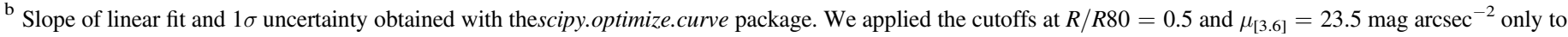

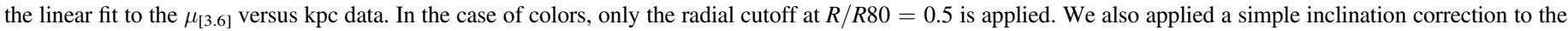
data by adding $-2.5 \log _{10}(b / a)$, where $a$ and $b$ are the semimajor and semiminor axes, respectively.

c The $y$ intercept of the linear fit with uncertainty.

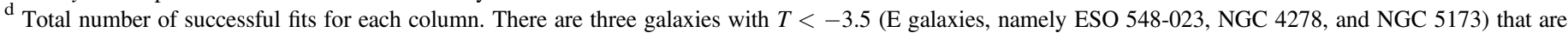
included in the $\mu_{[3.6]}$ versus kpc column, bringing the total to 992 galaxies, but they are removed from the subsample for further analysis.

(This table is available in its entirety in machine-readable form.)

in their outskirts, or alternatively, a recent enhancement of the SFR in the inner regions of an otherwise passively evolving system. In the latter case, the low fraction of intermediate-type spirals in the GRS (compared to the GGV) suggests that this rebirth should be accompanied by a morphological transformation from ETGs toward later galaxy types. There are, indeed, post-starburst $(\mathrm{E}+\mathrm{A})$ or $(\mathrm{K}+\mathrm{A})$ galaxies that are in the classical Green Valley (French et al. 2015) that did have centrally concentrated star formation (Norton et al. 2001).

In the more likely case of a decline of the SFR in the outer disks of GGV intermediate-type spirals, we should invoke the presence of a quenching (or, at least, damping) mechanism for the star formation acting primarily in these regions.

Figure 10 shows the color profiles of (FUV - NUV), (FUV - [3.6]), and (NUV - [3.6]) versus $\mu_{[3.6]}$ surface brightness. Linear fits to these color profiles were performed for each individual galaxy and are included in Table 7. The fits were performed for SB fainter than $\left.\mu_{[} 3.6\right]=20.89 \mathrm{mag} \operatorname{arcsec}^{-2}$ in these cases.

While a positive gradient seems to be more pronounced in (FUV - NUV) color compared to the other two, it is not clear what is driving it. Since dust reddening is rarely increasing toward the outer parts, those objects with positive (FUV - NUV) color gradients are likely suffering changes in the recent star formation history of their outer regions. The dominant morphological types of positive (FUV - NUV) color gradient galaxies are $\mathrm{S} 0$-a galaxies.

The comparison between the (NUV - [3.6]) and (FUV - [3.6]) color profiles (both shown in Figure 10) is also important to determine whether the UV emission is coming from newly formed $\mathrm{O}$ and $\mathrm{B}$ stars or from evolved UV-upturn sources (likely associated with EHB stars for the latter case, which mainly contribute to the FUV band; O'Connell 1999; see also Section 4.4).

\subsection{Color-color Diagrams}

From the colors measured above, we formed three colorcolor diagrams, namely (FUV - NUV) versus (NUV - [3.6]) (Figure 11), (FUV - NUV) versus (FUV - [3.6]) (Figure 12), and (FUV - [3.6]) versus (NUV - [3.6]) (not shown). The color-color diagrams presented here show the galaxies separated into nine panels of separate morphological type.

Comparing the (FUV - NUV) versus (NUV - [3.6]) and the (FUV - NUV) versus (FUV - [3.6]) color-color diagrams, we can see that the two sequences are more distinguishable in the former. This is mainly caused by the fact that the GRS is orthogonal to the GBS in the case of the (FUV - NUV) versus (NUV - [3.6]) diagram. This is due to the fact that the strength of the UV upturn also increases with the stellar mass surface density. This is also the case when considering the total galaxy mass (Boselli et al. 2005). We cannot determine here whether this is due to the stellar populations at high stellar mass surface densities hosting either an important helium rich or metal-poor horizontal branch (HB) population (Yi et al. 2005, 2011) or whether it is related to changes in the IMF (as suggested by Zaritsky et al. 2014b, 2015). 

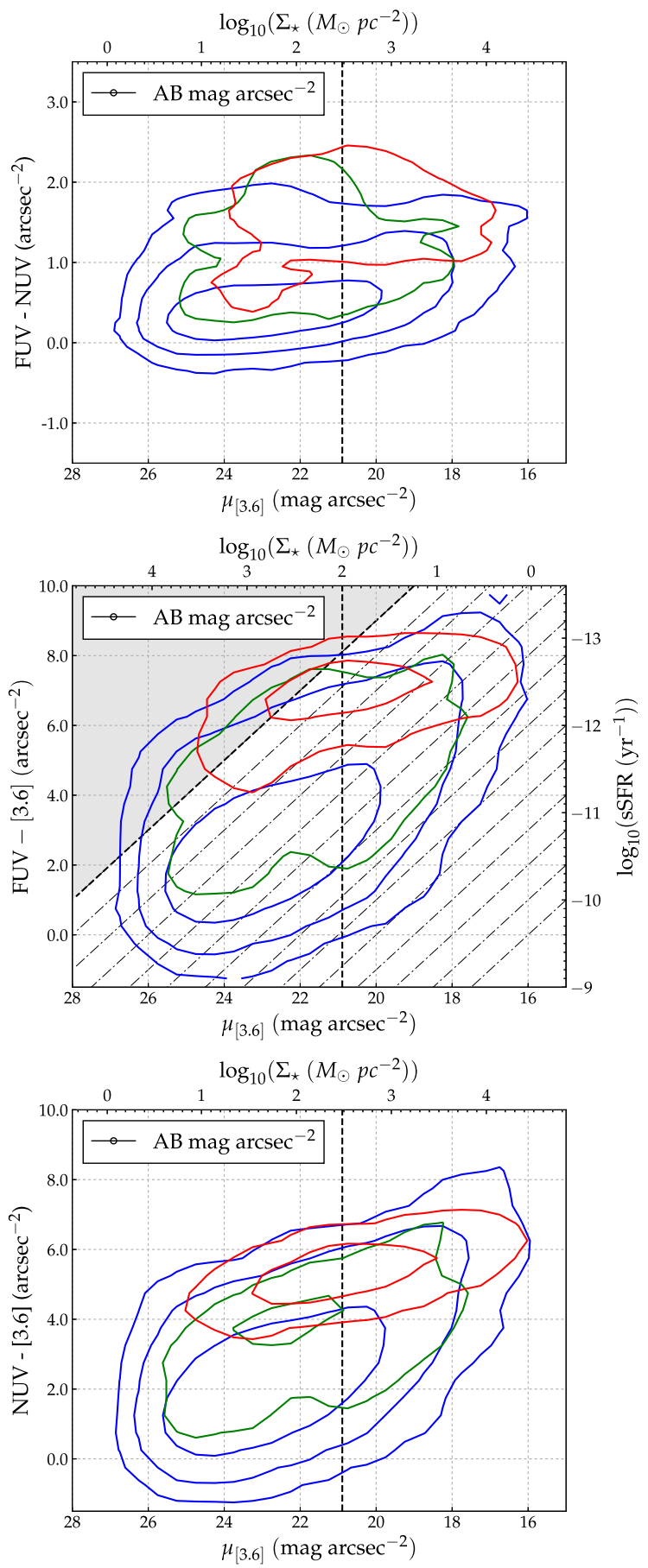

Figure 10. The (FUV - NUV), (FUV - [3.6]), and (NUV - [3.6]) colors vs. $\mu_{[3.6]}$ surface brightness profile contours for GBS, GGV, and GRS galaxies. Contours levels are slightly smoothed with a Gaussian kernel and describe the number density of SB profile data points. The outermost level corresponds to number densities of 0 dex (i.e., at least one data point) in each 2D bin (the binning is $0.1 \mathrm{mag}$ for (FUV - NUV) color and $0.5 \mathrm{mag}$ for everything else). Then, each contour level corresponds to an increase in number density by 1 dex. In the case of the (FUV - [3.6]) vs. $\mu_{[3.6]}$ diagram, diagonal lines represent constant FUV surface brightness $\mu_{\mathrm{FUV}}$, with the leftmost dashed line corresponding to $\mu_{\mathrm{FUV}}=29 \mathrm{AB}$ mag arcsec ${ }^{-2}$ (at the gray boundary) and decreasing by unity for each diagonal to the right. These are equivalent to lines of constant observed SFR surface density. The vertical black dashed line corresponds to $\log _{10}\left(\Sigma_{\star}\left(M_{\odot} \mathrm{pc}^{-2}\right)\right)=2.477\left(\right.$ or $\mu_{[3.6]}=20.89 \mathrm{mag} \operatorname{arcsec}^{-2}$; Kauffmann et al. 2006). The entire sample is shown in this case.

The (FUV - NUV) versus (NUV - [3.6]) color-color diagram is where we defined the GBS, GRS, and GGV subsamples from the galaxies' integrated (asymptotic) magnitudes by visually separating the distribution into two regions and fitting an error-weighted least-squares line to each region (Bouquin et al. 2015). With our current spatially resolved data, we can see the spatially resolved (radially, at least) color evolution of galaxies in these three categories. While ETGs such as E, E-S0, S0, S0-a, and Sa galaxies span both the GBS and GRS regions, LTGs such as $\mathrm{Sb}, \mathrm{Sc}, \mathrm{Sd}, \mathrm{Sm}$, and Irregular galaxies have this color much more constrained and their entire profile mostly located within the GBS region (mean $\pm 2 \sigma$ ).

In the panels for the E, E-S0, S0, and S0-a types (top row) of the (FUV - NUV) versus (NUV - [3.6]) (Figure 11) and (FUV - NUV) versus (FUV - [3.6]) (Figure 12) color-color diagrams, the galaxies are distributed into two regions: the bottom left (blue-blue) and the top right (red-red) parts in both color-color diagrams. The galaxies with the bluest central region have redder disks in (FUV - NUV), (NUV - [3.6]) and (FUV - [3.6]) colors. The galaxies with the reddest central region also have redder disks in (FUV - NUV), but not much in (NUV - [3.6]) nor in (FUV - [3.6]). In both cases, their central regions (triangles) are bluer in (FUV - NUV) color than their outer parts. If the bluing were caused by residual star formation (RSF), which contributes in both FUV and NUV, the observed data points would be bluer in all three colors. This is indeed the case for the ETGs seen in the bottom left (well within the GBS) in both color-color diagrams, where RSF is more prominent in their central regions. Note that the innermost $6^{\prime \prime}$ (in semimajor axis, i.e., 12" in major axis) are excluded, so the potential contribution of AGNs should not affect these results in a direct way.

For the ETGs in the top right of these plots, there is a difference between the (NUV-[3.6]) and (FUV - [3.6]) colors. While in the (FUV - NUV) versus (NUV - [3.6]) color-color diagram the distribution of these reddest systems has a negative slope (which provides a better isolation of the GRS), it has a positive slope in the (FUV - NUV) versus (FUV - [3.6]) color-color diagram. The central regions of these galaxies are bluer in (FUV - [3.6]) than in (NUV - [3.6]), which is the sign of a weaker contribution from the emitter of the UV radiation in these systems in the NUV than in the FUV compared to GBS galaxies. This can probably be attributed to evolved (UV-upturn) stars.

Our color-color diagrams are, thus, able to segregate and allow us to extract the properties of a whole range of galaxies, from star-forming LTGs to ETGs with and without RSF. For ETGs, they allow us to directly see the effect of UV-upturn stars, which can only be done in the UV-to-IR colors. In this regard, we find that RSF in ETGs seems to be concentrated in the center and the UV upturn is also stronger as we move to the inner regions of red (in NUV - [3.6]) ETGs. However, it should be noted that a recent study by Yildiz et al. (2017) showed that a not-insignificant fraction, 20\%, of field (non-Virgo) nearby galaxies have disks or rings of $\mathrm{HI}$ gas around them, and that their UV profiles are closely tied to their $\mathrm{H}$ I gas reservoir.

This color-color diagram does not allow us to clearly determine whether the UV upturn is also present in the bulges of early-type spiral galaxies as they are located in a position similar to that expected for turnoff stars in these bulges. We can nevertheless conclude that in galaxies with morphological types later than $\mathrm{Sc}$, the light from HB stars is clearly overshone by these turnoff stars of progressively higher masses (statistically speaking) as we move to later types. 
Table 7

Gradients of Color vs. $3.6 \mu \mathrm{m}$ Surface Brightness Profiles

\begin{tabular}{|c|c|c|c|c|c|c|}
\hline \multirow{3}{*}{$\begin{array}{l}\text { Unit } \\
\text { Name }^{a}\end{array}$} & \multirow{2}{*}{\multicolumn{2}{|c|}{$\frac{(\mathrm{FUV}-\mathrm{NUV}) / \mu_{[3.6]}}{\mathrm{mag} /\left(\mathrm{mag} \operatorname{arcsec}^{-2}\right)}$}} & \multirow{2}{*}{\multicolumn{2}{|c|}{$\frac{(\mathrm{FUV}-[3.6]) / \mu_{[3.6]}}{\mathrm{mag} /\left(\mathrm{mag} \operatorname{arcsec}^{-2}\right)}$}} & \multirow{2}{*}{\multicolumn{2}{|c|}{$\frac{(\mathrm{NUV}-[3.6]) / \mu_{[3.6]}}{\mathrm{mag} /\left(\mathrm{mag} \operatorname{arcsec}^{-2}\right)}$}} \\
\hline & & & & & & \\
\hline & $a^{\mathrm{b}}$ & $b^{\mathrm{c}}$ & $a$ & $b$ & $a$ & $b$ \\
\hline UGC 00017 & $-0.03 \pm 0.08$ & $0.94 \pm 1.92$ & $-0.69 \pm 0.18$ & $18.83 \pm 4.39$ & $-0.63 \pm 0.10$ & $17.21 \pm 2.30$ \\
\hline ESO 409-015 & $0.21 \pm 0.03$ & $-4.78 \pm 0.64$ & $0.93 \pm 0.07$ & $-21.89 \pm 1.64$ & $0.72 \pm 0.05$ & $-17.13 \pm 1.08$ \\
\hline ESO 293-034 & $-0.01 \pm 0.02$ & $0.79 \pm 0.48$ & $-0.40 \pm 0.04$ & $11.81 \pm 0.88$ & $-0.42 \pm 0.03$ & $11.61 \pm 0.62$ \\
\hline NGC 0210 & $-0.09 \pm 0.04$ & $2.27 \pm 0.96$ & $-1.01 \pm 0.18$ & $26.28 \pm 4.03$ & $-0.79 \pm 0.13$ & $20.81 \pm 2.84$ \\
\hline ESO 079-005 & $-0.04 \pm 0.03$ & $1.33 \pm 0.75$ & $-0.40 \pm 0.10$ & $10.96 \pm 2.41$ & $-0.40 \pm 0.08$ & $10.69 \pm 1.76$ \\
\hline NGC 0216 & $0.26 \pm 0.01$ & $-5.20 \pm 0.22$ & $0.39 \pm 0.03$ & $-5.96 \pm 0.74$ & $0.13 \pm 0.03$ & $-0.74 \pm 0.60$ \\
\hline PGC 002492 & $-0.09 \pm 0.03$ & $2.30 \pm 0.64$ & $-0.50 \pm 0.05$ & $13.43 \pm 1.23$ & $-0.46 \pm 0.05$ & $12.20 \pm 1.18$ \\
\hline IC 1574 & $0.19 \pm 0.05$ & $-4.10 \pm 1.20$ & $0.64 \pm 0.16$ & $-13.28 \pm 3.86$ & $0.41 \pm 0.13$ & $-8.35 \pm 2.98$ \\
\hline NGC 0244 & $0.24 \pm 0.09$ & $-4.96 \pm 1.99$ & $0.38 \pm 0.03$ & $-6.72 \pm 0.75$ & $0.13 \pm 0.06$ & $-1.45 \pm 1.32$ \\
\hline PGC 002689 & $-0.08 \pm 0.05$ & $1.99 \pm 1.13$ & $-0.08 \pm 0.19$ & $2.80 \pm 4.53$ & $0.03 \pm 0.16$ & $0.11 \pm 3.78$ \\
\hline UGC 00477 & $-0.04 \pm 0.05$ & $1.17 \pm 1.21$ & $-0.68 \pm 0.06$ & $17.59 \pm 1.43$ & $-0.63 \pm 0.03$ & $16.10 \pm 0.71$ \\
\hline ESO 411-013 & $-0.25 \pm 0.15$ & $6.24 \pm 3.58$ & $-0.50 \pm 0.07$ & $13.91 \pm 1.60$ & $-0.35 \pm 0.19$ & $10.11 \pm 4.56$ \\
\hline NGC 0247 & $-0.06 \pm 0.02$ & $1.76 \pm 0.51$ & $-0.23 \pm 0.11$ & $7.87 \pm 2.33$ & $-0.10 \pm 0.09$ & $4.73 \pm 1.95$ \\
\hline Total $^{\mathrm{d}}$ & 1650 & & 1650 & & 1650 & \\
\hline
\end{tabular}

Notes.

${ }^{\text {a }}$ Same nomenclature as the $\mathrm{S}^{4} \mathrm{G}$. Sorted by R.A.

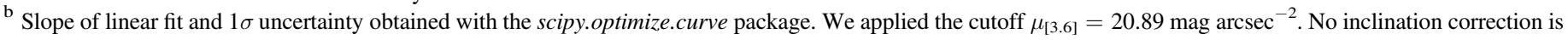
applied in these cases.

c The $y$ intercept of the linear fit with uncertainty.

d Total number of successful fits for each column.

(This table is available in its entirety in machine-readable form.)

Table 8

GALEX $/ \mathrm{S}^{4} \mathrm{G}$ Sample Radial Ranges at $3.6 \mu \mathrm{m}$

\begin{tabular}{lllllr}
\hline \hline Morph $^{\mathrm{a}}$ & $N^{\mathrm{b}}$ & $\begin{array}{c}R 80 \text { range }^{\mathrm{c}} \\
\mathrm{kpc}\end{array}$ & $\begin{array}{c}\text { max range } \\
\mathrm{kpc}\end{array}$ & $\begin{array}{c}\langle R 80\rangle^{\mathrm{e}} \\
\mathrm{kpc}\end{array}$ & $\begin{array}{c}\langle\mathrm{max}\rangle^{\mathrm{f}} \\
\mathrm{kpc}\end{array}$ \\
\hline E & 24 & $1.86-18.79$ & $6.51-55.39$ & 6.51 & 23.77 \\
E-S0 & 23 & $0.94-12.2$ & $3.23-42.98$ & 3.60 & 14.00 \\
S0 & 51 & $1.01-12.22$ & $3.71-45.47$ & 3.98 & 16.57 \\
S0-a & 103 & $1.43-15.14$ & $5.44-51.54$ & 4.67 & 18.26 \\
Sa & 175 & $1.14-17.31$ & $6.33-57.03$ & 5.26 & 21.38 \\
Sb & 340 & $0.97-17.21$ & $4.2-90.17$ & 5.77 & 22.27 \\
Sc & 669 & $0.88-19.57$ & $2.25-81.73$ & 6.22 & 19.21 \\
Sd & 168 & $1.24-15.68$ & $2.48-39.79$ & 5.53 & 13.55 \\
Sm & 192 & $0.18-12.54$ & $0.32-36.04$ & 4.94 & 11.68 \\
Irr & 186 & $0.19-17.59$ & $0.31-28.86$ & 4.01 & 8.61 \\
\hline
\end{tabular}

Total 1931

Notes.

${ }^{\text {a }}$ C2 morphological types.

b Number of galaxies.

${ }^{c}$ Smallest and largest $R 80$ distance in kiloparsec.

d Smallest and largest maximum size of galaxies in kiloparsec.

e Average $R 80$.

${ }^{f}$ Average maximum.

\subsection{GGV Galaxies}

A subsample of $70 \mathrm{GGV}$ galaxies was identified in the (FUV - NUV) versus (NUV - [3.6]) integrated color-color diagram by Bouquin et al. (2015).

As already pointed out in that paper, these objects can be interpreted as galaxies that have either left the GBS and are "transitioning" to eventually reach the GRS or were previously in the GRS and are now experiencing a modest rebirth or rejuvenation (in terms of the light-weighted ages of their stellar populations) and are evolving back to the GBS.

In the former scenario, star formation would have been suppressed (or at least damped) by starvation from having used up all the gas, ram-pressure stripping, or quenching due to the perturbations induced from AGNs, merger events, or some other gas-heating process. OB stars would not form any longer, and the FUV and NUV emissions would decrease, with the FUV emission evolving faster than the NUV because of the shorter lifespan of the most massive stars, resulting in a progressive reddening of their (FUV - NUV) color.

In the case of the latter (rejuvenation) scenario, these galaxies would have started to form stars on top of relatively passively evolving galaxies either by the accretion of new gas or by cooling gas that was already present in the galaxy in a hotter phase.

The results presented above provide another fundamental piece of evidence for the origin of these transitioning objects. In particular, we have shown that the outer parts of most GGV galaxies are redder than their inner parts and that this reddening is progressive (see, e.g., Figure 13). In the case of the quenching scenario, this implies that the mechanism responsible for the quenching is acting in an outside-in fashion. Should the rejuvenation scenario be happening, these galaxies would be starting to form stars from the inside out. As the associated blue colors are not limited to the very central regions, this would likely imply the growth of a disk, again, in an inside-out fashion.

With regard to the mechanism(s) that could potentially lead to the suppression of star formation in the outskirts, we showed in Bouquin et al. (2015) that the GGV has the highest fraction of Virgo cluster galaxies, with 20 (out of 70) GGV objects in the Virgo cluster, i.e., $\sim 29 \%$, in comparison to a fraction of Virgo cluster galaxies in the GBS of only $~ 7 \%(124 / 1753)$ and 


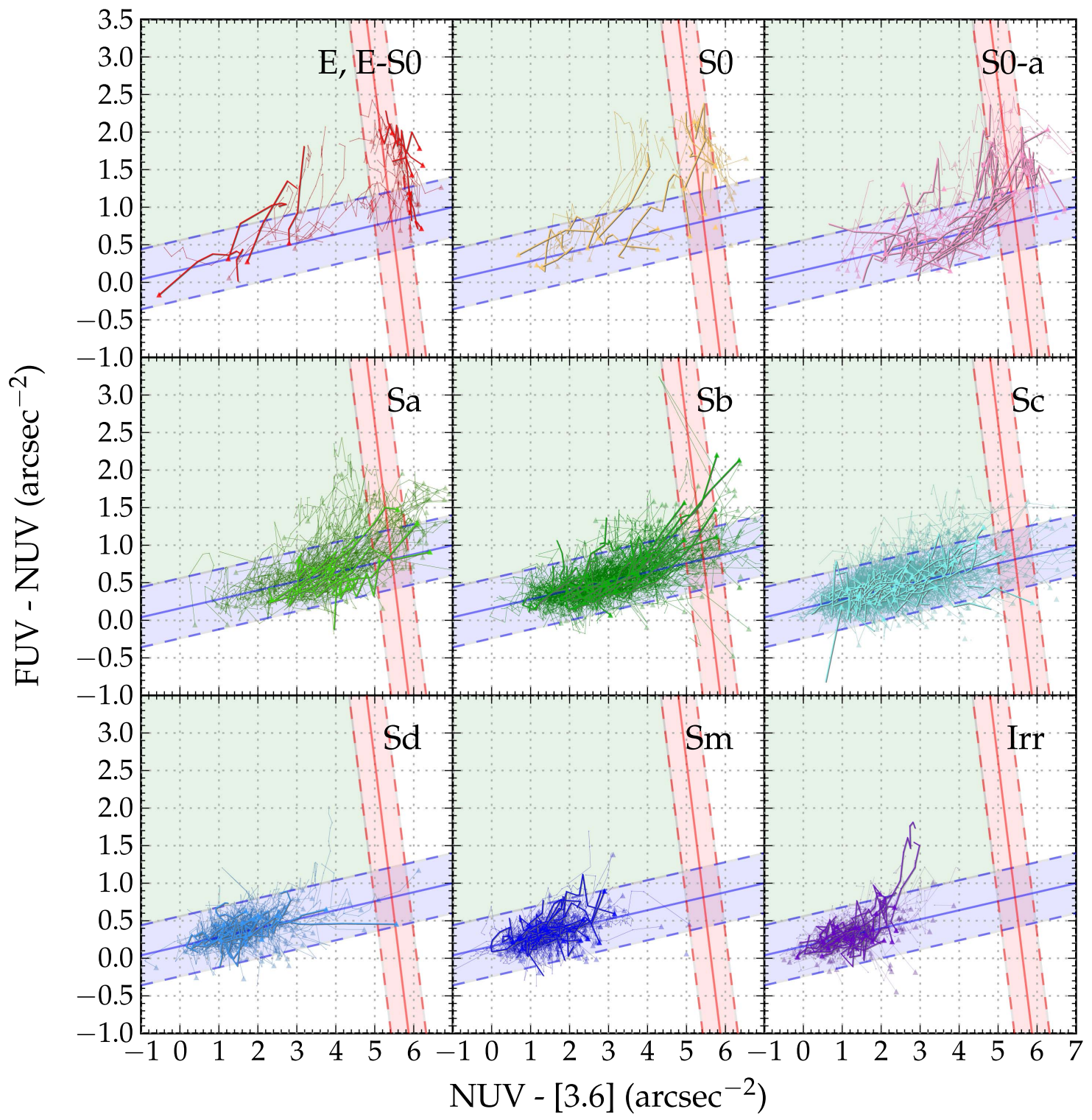

Figure 11. The (FUV - NUV) vs. (NUV - [3.6]) spatially resolved color-color diagrams per morphological type. The regions delineated by a solid line and two parallel dashed lines are the GBS in blue and the GRS in red, and the green region in the upper left quadrant is the GGV, as defined in Bouquin et al. (2015). Measurements at the center are represented by triangles, and other measurements, as we move radially outward every $6^{\prime \prime}$, are represented by dots connected by a line for each galaxy. Randomly selected galaxies are emphasized in each panel for better visualization.

in the GRS of $\sim 18 \%(14 / 79)$. For example, one ram-pressure model in Virgo (Boselli et al. 2006) creates an inverted color gradient compared to late-type field galaxies, with redder outer disks and bluer inner parts.

We also analyze whether the GGV objects are mainly located in groups where environmental effects might start to occur (in particular, strangulation; Kawata \& Mulchaey 2008). Among the $70 \mathrm{GGV}$ galaxies of our sample, $28(40 \%)$ are field galaxies and $42(60 \%)$ are in groups or clusters. We see that the fraction of field galaxies decreases to $30 \%$, while the fraction of group galaxies increases to $70 \%(56 / 79)$ in the case of GRS galaxies. In contrast, the fraction of field/group galaxies is $51 \% / 49 \%$ in the case of GBS galaxies, and that of the overall sample is $50 \% / 50 \%$. That is, we see an increase in the fraction of galaxies belonging to groups as we go from the GBS to the GRS. This result hints that the disk reddening that we see in GGV galaxies is likely due to a mechanism that is favored in dense environments. We note that this result does not exclude rejuvenation scenarios, as many ETGs with extended star formation are now being identified (Fang et al. 2012; Salim et al. 2012; Y1ldiz et al. 2017).

\section{Modeling 3.6 $\mu \mathrm{m}$ Exponential Disks}

The linear disk fits were compared to the profiles of BP00 disk models, generated with various circular velocities and spin parameters. These are simple disk models without any bulge, bar, or mass outflow features, calibrated on the MW with the assumption that our Galaxy is a typical spiral galaxy (Boissier \& Prantzos 1999, hereafter BP99) and using simple scaling relations to extend the initial model to other spirals (BP00). These models grow from the inside out with an infall of primordial gas (i.e., low metallicity) with a radially varying and exponentially decreasing infall rate with time. They include realistic yields and lifetimes from stellar evolution models and metallicity enhancement by 


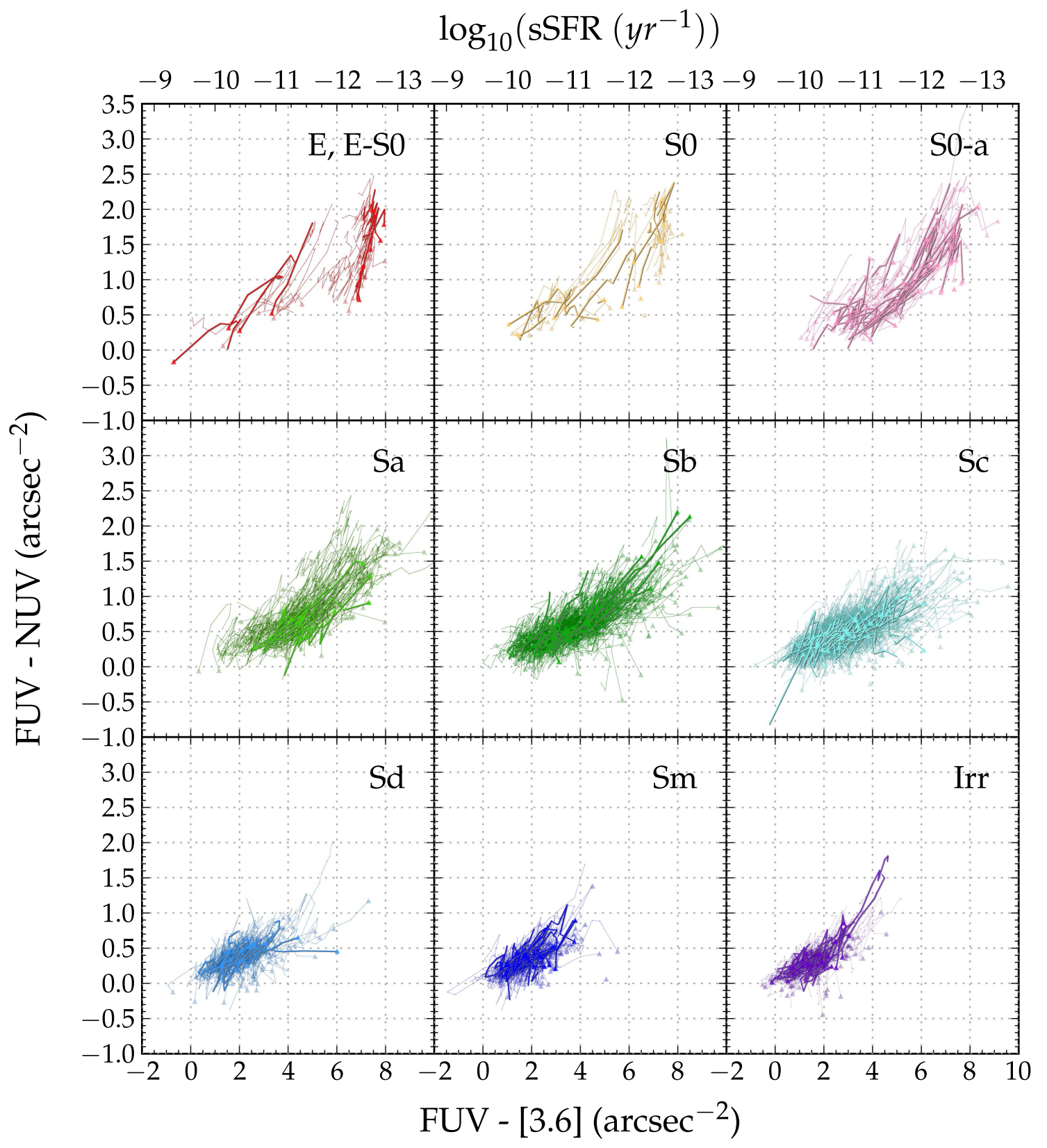

Figure 12. The (FUV - NUV) vs. (FUV - [3.6]) spatially resolved color-color diagrams per morphological type.

SNe Ia and adopt a Kroupa IMF. The local SFR varies with the gas surface density and angular velocity. The chemical and photometric evolution of the disk is then followed within this selfconsistent framework. The rotational velocity, $v_{\mathrm{c}}$, is related to the total baryonic mass (Mo et al. 1998) and is implemented in a relative way with respect to the MW model,

$$
\frac{v_{c}}{220}=\left(\frac{M}{M_{\mathrm{MW}}}\right)^{1 / 3},
$$

and the dimensionless spin parameter $\lambda$ is defined as (Peebles 1969)

$$
\lambda=J|E|^{1 / 2} G^{-1} M^{-5 / 2},
$$

where $M$ is the total baryonic mass, $M_{\mathrm{Mw}}$ is the total baryonic mass of the MW, $220\left(\mathrm{~km} \mathrm{~s}^{-1}\right)$ is the circular velocity of the MW, $J$ is the angular momentum, $E$ is the energy of the halo, and $G$ is the gravitational constant. In the BP00 models, the spin parameter only influences the scale length of the disk with respect to the MW,

$$
\frac{R}{R_{\mathrm{MW}}}=\frac{V}{V_{\mathrm{MW}}} \frac{\lambda}{\lambda_{\mathrm{MW}}},
$$

where $R$ and $\lambda$ are the scale length and spin parameter of the considered model and $R_{\mathrm{MW}}$ and $\lambda_{\mathrm{MW}}$ are those of the MW. We show that we are able to obtain circular velocities and spin for the galaxies of our sample from this method (Section 5.1). Finally, we show color gradients against the circular velocity, spin parameter, and stellar mass of our sample (Section 5.2). In particular, gradients are positive at $\sim 50 \mathrm{~km} \mathrm{~s}^{-1}$, the average is flat at $\sim 75 \mathrm{~km} \mathrm{~s}^{-1}$, and above $\sim 100 \mathrm{~km} \mathrm{~s}^{-1}$, most galaxies have negative gradients in all three colors. 

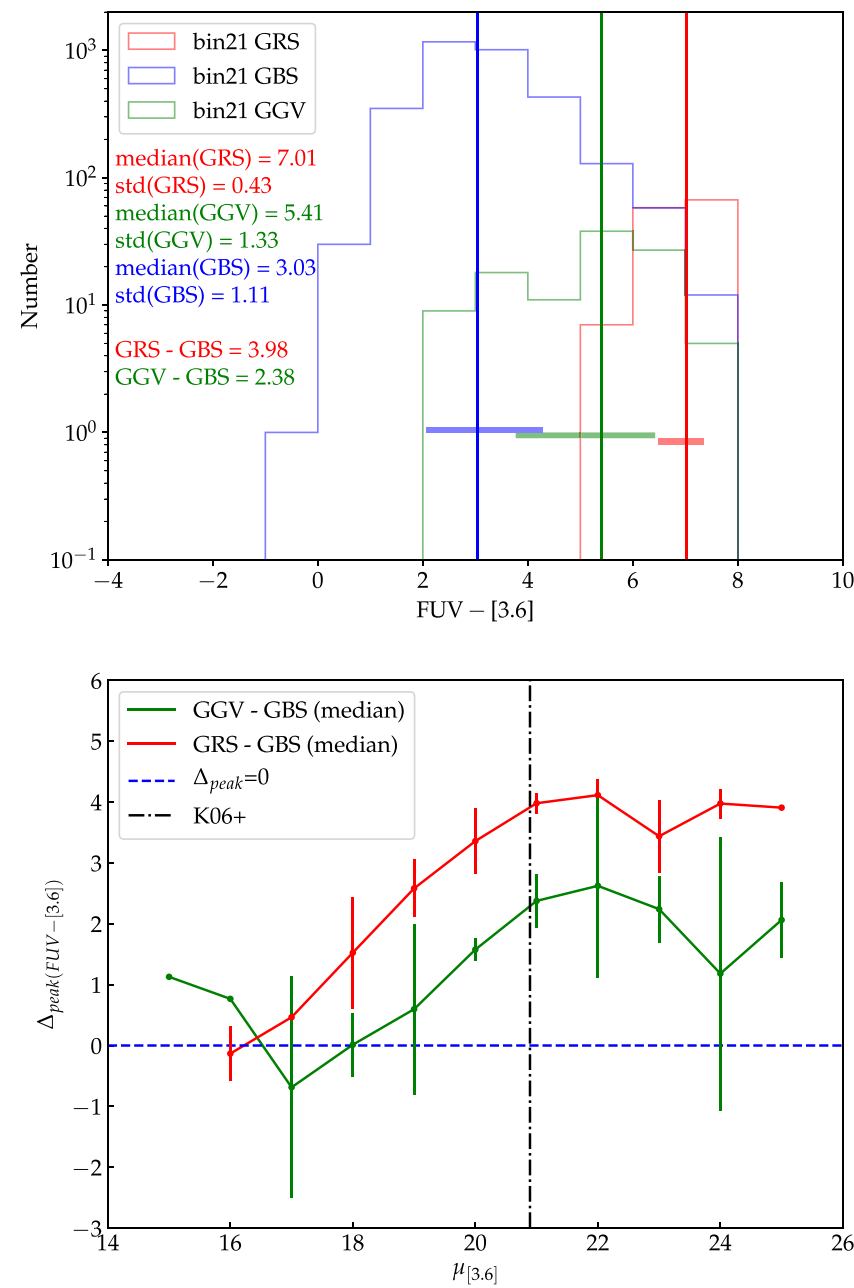

Figure 13. Top: number counts of data points of GBS (blue), GGV (green), and GRS (red) galaxies within a $3.6 \mu \mathrm{m}$ surface brightness bin $21 \leqslant \mu_{[3.6]}<22$ in the (FUV - [3.6]) color vs. $\mu_{[3.6]}$ surface brightness plot. Solid lines correspond to the median, and the horizontal shaded areas represent the extent of one standard deviation above and below the mean (not shown), all in their respective colors. Bottom: difference of the peak (FUV - [3.6]) color (i.e., the difference of the median) of the GRS and GBS distributions for each $\mu_{[3.6]}$ bin (red). The same is in green for the difference between the peaks of the distributions of GGV and GBS. The dashed blue line at $\Delta_{\text {peak }}=0$ and the dotdashed black line at $\mu_{[3.6]}=20.892 \mathrm{mag} \mathrm{arcsec}{ }^{-2}$ of Kauffmann et al. (2006) are shown for reference. Error bars represent the 15.865 and 84.135 percentiles of the GRS and GGV distributions obtained by using the IQR method.

\subsection{Obtaining Circular Velocity and Spin}

In this study, we use the disk models of BP00 as in the version presented in Muñoz-Mateos et al. (2011) but increasing the sampling and range spanned by the model parameters, namely circular velocity $v_{\mathrm{c}}$ and $\operatorname{spin} \lambda$. As mentioned above, these are bulgeless, disk-only models that naturally grow inside-out from gas infall and are left to run for $T=13.5 \mathrm{Gyr}$ to the present. They include scaling laws so that mass scales as $v^{3}$ and scale length as $\lambda \times v$ (Mo et al. 1998). As can be seen in Figure 14, an increase in circular velocity $v_{\mathrm{c}}$ leads to an increase in both the total stellar mass and the disk scale length, whereas increasing the spin parameter $\lambda$ only increases the scale length. Correcting our observed galaxies for inclination (see Section 4.1) leads to a dimming in surface brightness at all radii and thus eventually would yield a lower circular velocity and a larger spin than when not applying the correction.
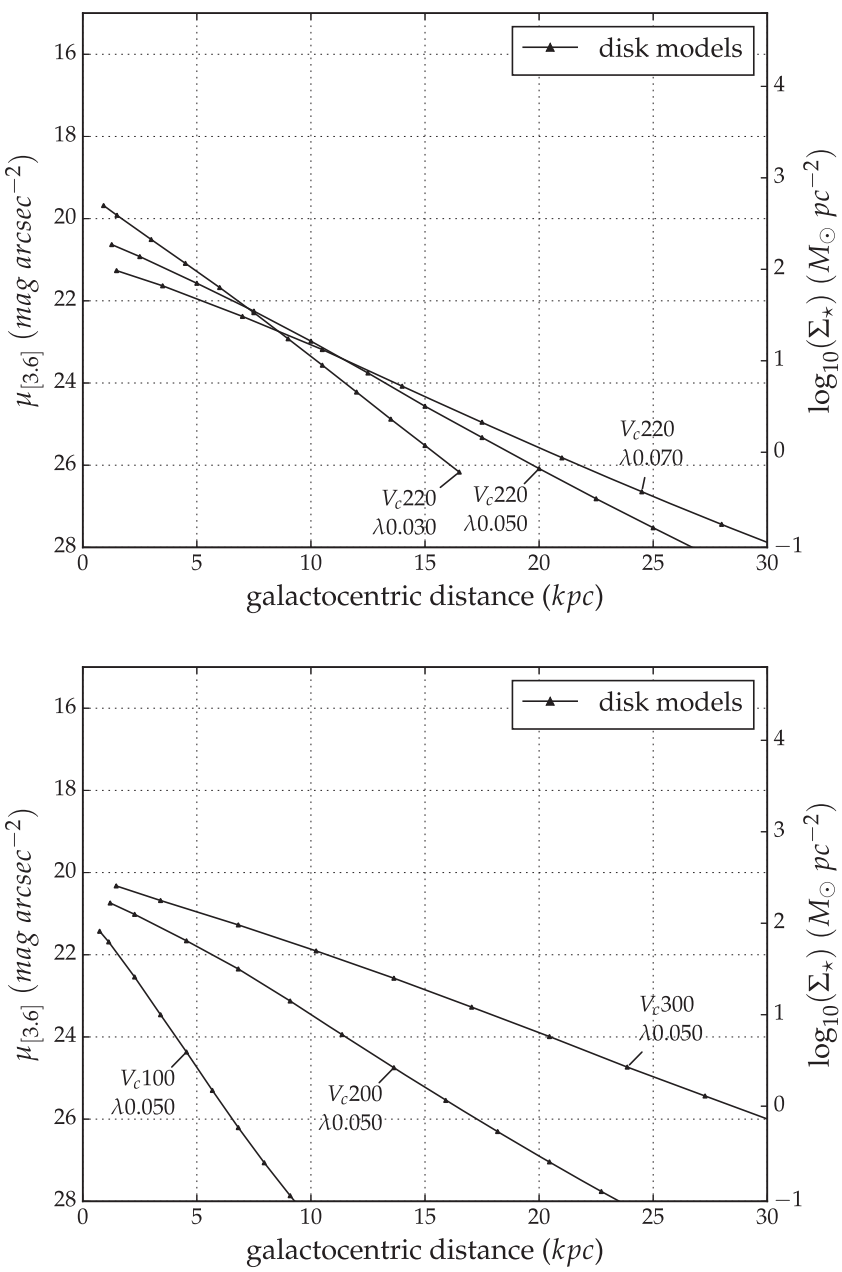

Figure 14. Examples of $3.6 \mu \mathrm{m}$ surface brightness profiles of BP00 disk models with fixed circular velocity $v_{\mathrm{c}}$ and variable spin parameter $\lambda$ (top) and with fixed spin parameter and variable circular velocity (bottom).

These models aimed to reproduce the multiwavelength SB profile by varying only those two parameters. Other assumptions were calibrated in the MW model (BP99) and in nearby disks (BP00). Predictions for disks with different spins and velocities are based on $\Lambda \mathrm{CDM}$ scaling laws. Disk models were generated for various spin parameters and circular velocity combinations: the spin ranges from 0.002 to 0.15 , inclusively, and varying by a step of 0.001 , i.e., 149 different spins, while the velocity ranges from 20 to $430 \mathrm{~km} \mathrm{~s}^{-1}$, inclusively, and varying by a step of $10 \mathrm{~km} \mathrm{~s}^{-1}$, i.e., 42 different velocities. The total number of models generated is 6258. We fit these models with an error-weighted linear fit (in surface brightness scale) in a similar manner to what we do with our data points. It is, however, necessary to insert an uncertainty on the data point of each model in order to compute the reduced $\chi^{2}$ of the fit and to determine whether an exponential law also properly describes the radial distribution of the UV through near-infrared light in these models. A reasonable assumption in this regard is $0.10-0.15 \mathrm{mag}$ (see, e.g., Muñoz-Mateos et al. 2011). Indeed, a value of 0.15 mag yields a reduced $\chi^{2}$ close to unity for most of the models.

Figure 15 shows the slopes and $y$ intercepts obtained from the fits to the IR surface brightness profiles (corrected for inclination) of our galaxy sample plotted along with the grid of slopes and $y$ intercept obtained from fits to the BP00 models described above. Data error bars are coming from the slope and 


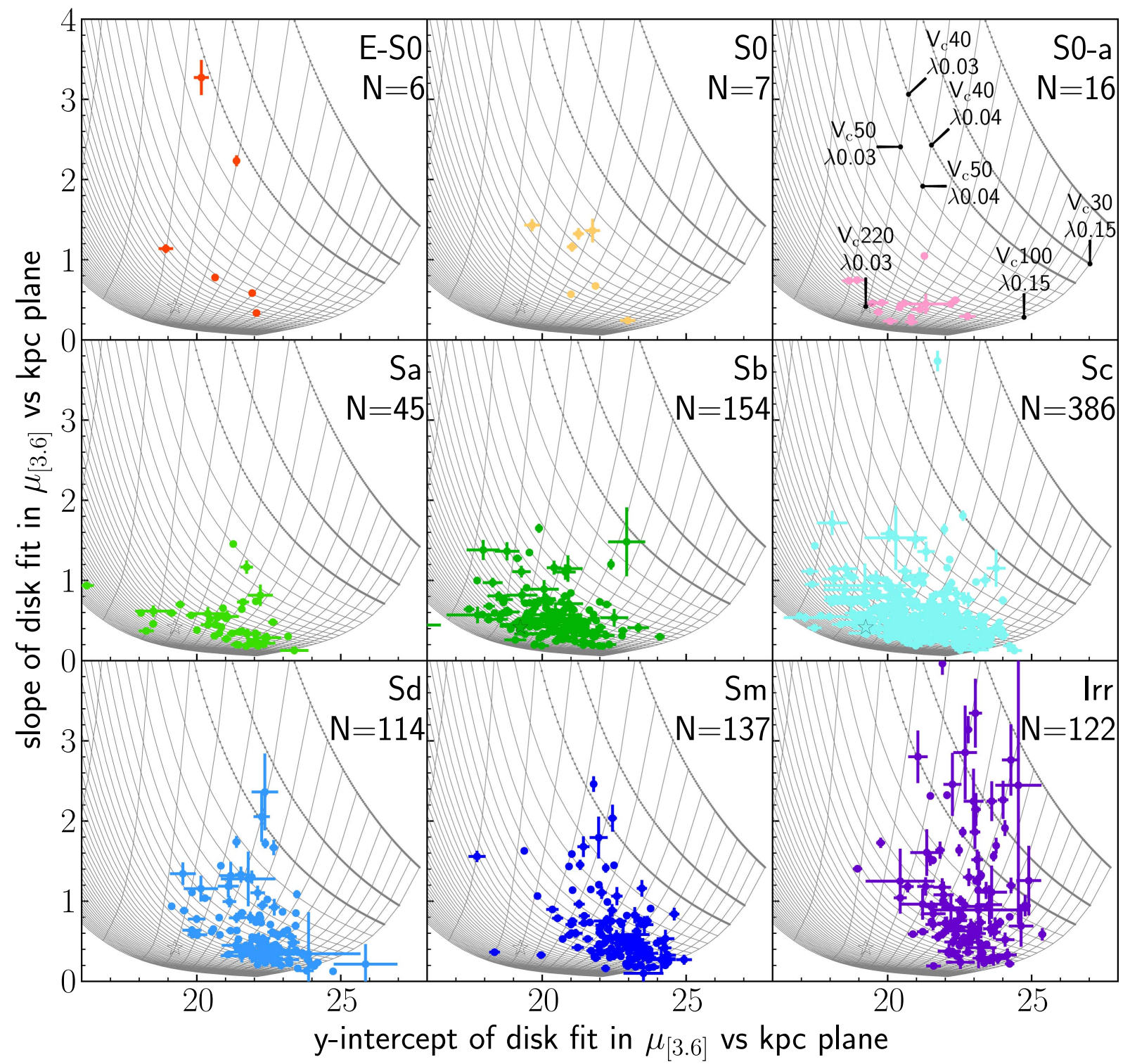

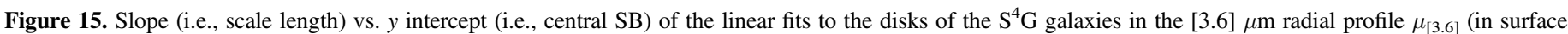

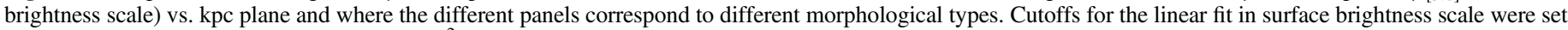

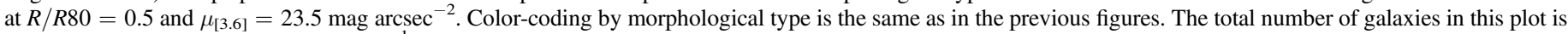

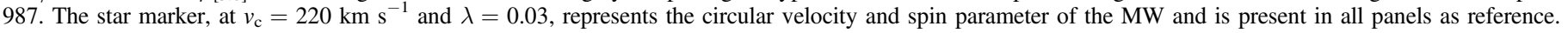

$y$-intercept fitting errors obtained from the weighted fits to our surface brightness profiles. The error bars in the slopes and $y$ intercepts of the models are omitted for simplicity. They are separated by morphological type.

This approach allows us to assign to a given galaxy disk a specific $3.6 \mu \mathrm{m}$ central surface brightness and scale length along with the corresponding closest model. That way, we are able to deduce circular velocities and spin parameters for the entire $S^{4} G$ sample. In Figure 16, we show the circular velocity and spin distributions and the comparison between both parameters for the entire sample. We split these parameters by morphological type.

For each pair of best-fitting slope (i.e., scale-length) and $y$ intercept (i.e., central surface brightness) measurements, we generated 1000 random points using elliptical 2D Gaussian probability distribution functions with $1 \sigma$ being the uncertainties in these measurements and obtained the closest model for each Monte Carlo particle. Thus, for each data point (i.e., for each galaxy), we obtained a distribution in best-fitting circular velocity and spin parameter. Typical distributions of circular velocities from the sampling of 1000 points are shown in Figure 17. This figure also shows the distribution of the individual 1000 points in the circular velocity versus spin diagram for three example galaxies. There is a mild degeneracy between the two parameters (although we show galaxies with very skewed distributions) in some of these objects that is in the same direction as the correlation seen in Figure 16 for late-type galaxies. Note, however, that such a correlation is not driving the whole distribution of points in Figure 16 and that the latter spans a wider range of spins and circular velocities than the $1 \sigma$ errors found for the individual galaxies. Thus, although the degeneracy between the two parameters certainly contributes to the morphology of the different panels of Figure 16, it also reflects the bona fide distribution of the physical properties of the disks of galaxies in the local universe. 

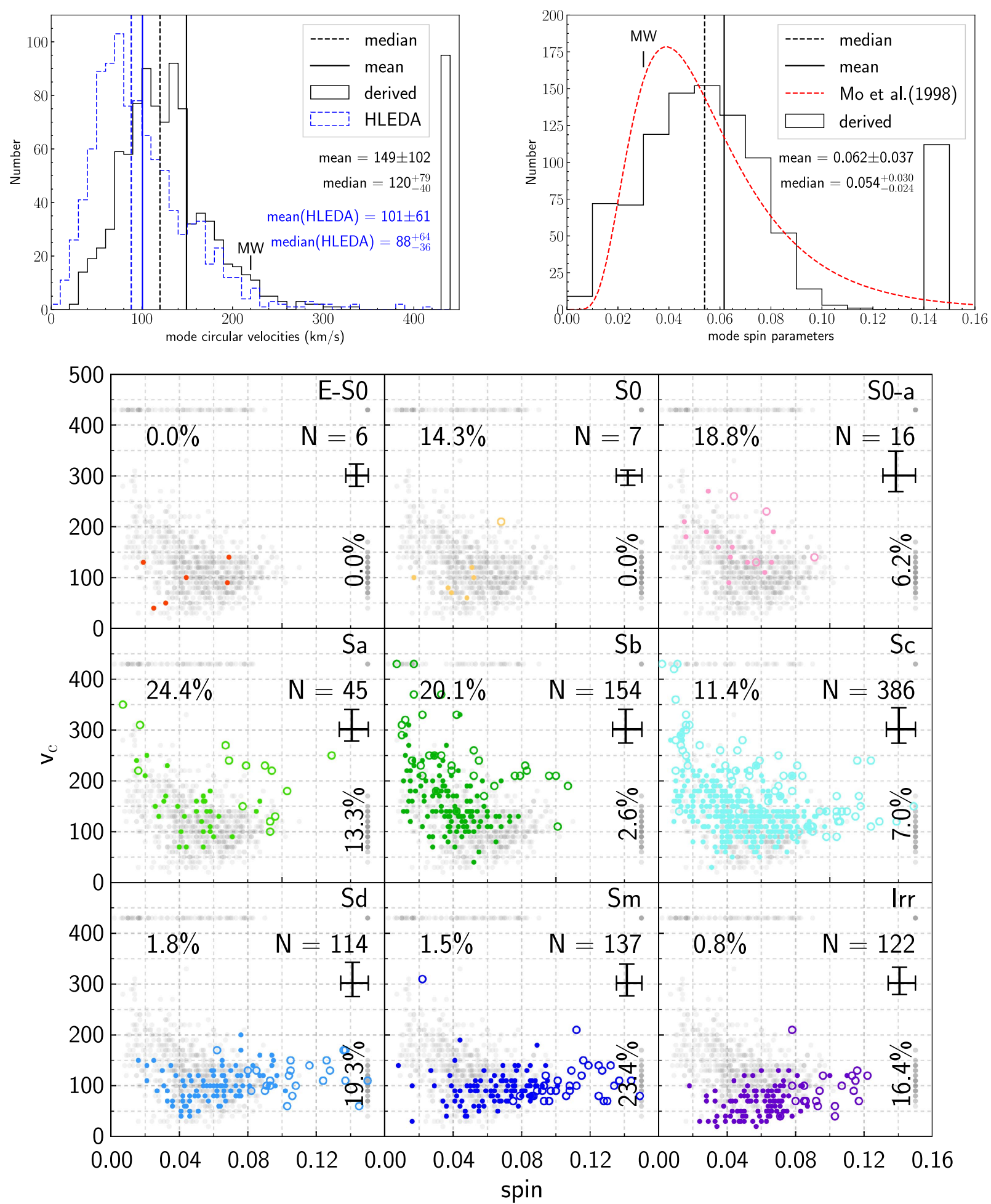

Figure 16. Distribution of the mode of the best-fitting models' circular velocity $v_{\mathrm{c}}$ (top left) and spin parameter $\lambda$ (top right) for the $\mathrm{S}^{4} \mathrm{G}$ sample when cutoffs of $R / R 80=0.5$ and $\mu_{[3.6]}=23.5 \mathrm{mag} \operatorname{arcsec}^{-2}$ are used to isolate the disk component of these galaxies' profiles. For the spin parameter distribution, we also show the probability distribution (scaled to our distribution so that both distributions have the same area) of the spin parameter derived by Mo et al. (1998) for comparison (red dashed line). For the circular velocity distribution, we compare it with circular velocities obtained from HyperLeda (blue dashed histogram). The deduced circular velocity distributions (black solid lines) are compared with the circular velocities available in HyperLeda (blue dashed lines). The mean (solid vertical line) and median (dashed vertical line) positions are shown for each distribution, annotated with the value and the $1 \sigma$ uncertainty. The circular velocity plotted against the spin parameter, split by morphological type, is shown in the bottom panel. Average uncertainties are shown in the top right corner. The MW values are shown in both panels. Open circles indicate galaxies that have extreme values in circular velocity, spin parameter, or both and are shown using their central value instead. The percentage shown in the upper left is the fraction of outliers in $v_{\mathrm{c}}$, whereas the one shown in the lower right and rotated is the fraction of outliers in spin parameters. Outliers in both $v_{\mathrm{c}}$ and spin are included in both fractions. 

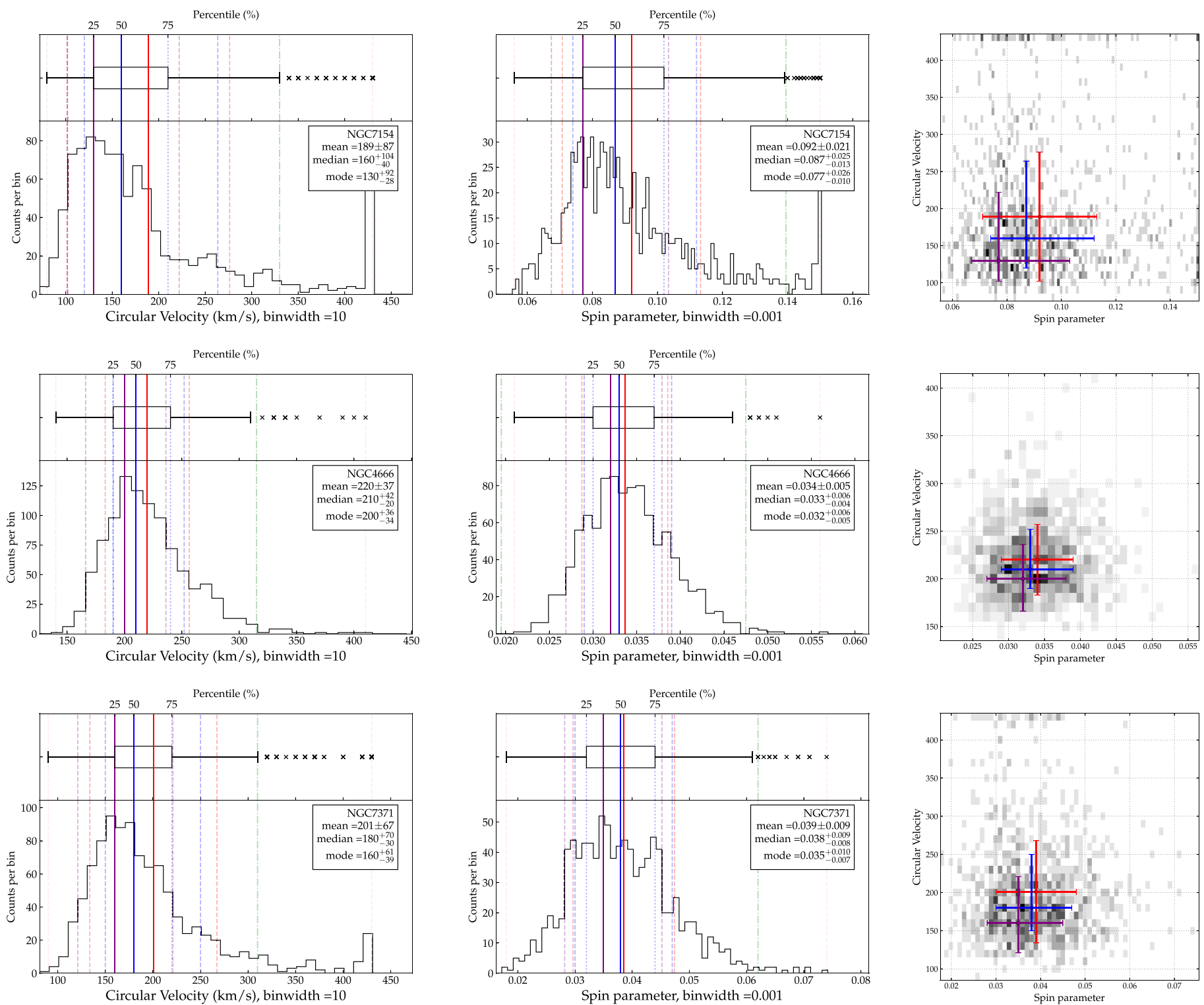

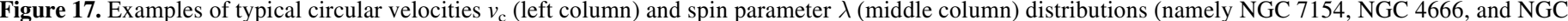

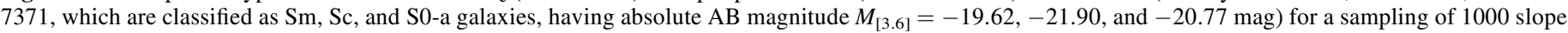

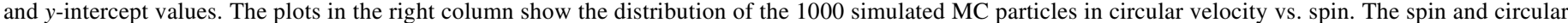

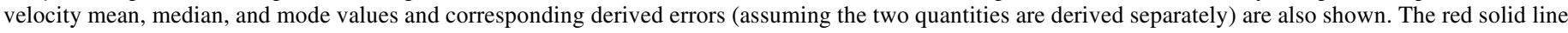

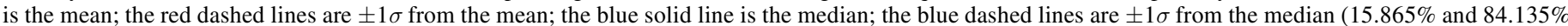

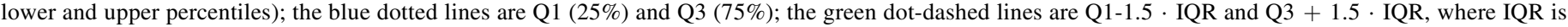

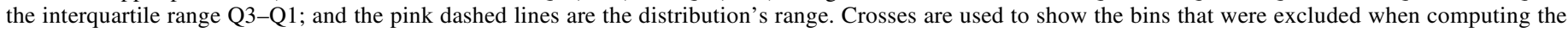
percentiles.

We see that this method of sampling produces circular velocity distributions with long tails toward high $v_{\mathrm{c}}$. These asymmetric distributions, for which the median or mode (rather than the mean) gives a better estimate of the peak of the distribution for the corresponding parameter, are a consequence of the nonregularity of the coverage of the model grid in Figure 15. In this work, we make use of the mode values and the percentiles obtained from these distributions to get the data points and average error bars in Figure 16. We also list the results obtained in Table 9.

We then compare our values with the observed values of the circular velocity for galaxies for which we have data (see
Figure 18). We obtained the inclination-corrected maximum rotational (i.e., circular) velocity and its associated uncertainty from HyperLeda, vrot and $e_{-} v$ rot. These observed values are computed from the apparent maximum rotation velocity obtained from the width of the $21 \mathrm{~cm}$ line at various levels or from $\mathrm{H} \alpha$ rotation curves. They are homogenized using a large sample $(>50,000)$ of measurements and are corrected for inclination (Paturel et al. 2003). We do not aim here to provide a fully coherent set of circular velocity measurements but to see whether or not the values that we obtain from our method are similar to the observed ones. In the case of our "best" $\chi^{2}$ fit with cutoffs of $R / R 80 \simeq 0.5$ and $\mu_{[3.6]} \simeq 23.5 \mathrm{mag} \mathrm{arcsec}{ }^{-2}$, 976 galaxies when using the median and 978 when using the 
Table 9

GALEX $/ \mathrm{S}^{4} \mathrm{G}$ Sample Circular Velocity and Spin Obtained from a Grid of BP00 Disk Models

\begin{tabular}{lrrr}
\hline \hline Galaxy Name $^{\mathrm{a}}$ & \multicolumn{1}{c}{$v_{\mathrm{c}}^{\mathrm{b}}$} & $\lambda^{\mathrm{c}}$ & \multicolumn{1}{c}{$T^{\mathrm{d}}$} \\
\hline ESO 293-034 & $130_{-28}^{+40}$ & $0.041_{-0.007}^{+0.008}$ & 6.2 \\
NGC 0024 & $110_{-27}^{+13}$ & $0.027_{-0.006}^{+0.003}$ & 5.1 \\
ESO 293-045 & $90_{-16}^{+24}$ & $0.066_{-0.009}^{+0.009}$ & 7.8 \\
UGC 00122 & $70_{-25}^{+15}$ & $0.067_{-0.007}^{+0.008}$ & 9.6 \\
NGC 0059 & $50_{-}^{+\ldots}$ & $0.032_{-0.004}^{+0.004}$ & -2.9 \\
ESO 539-007 & $110_{-45}^{+25}$ & $0.150_{-0.029}^{+\ldots .3}$ & 8.7 \\
ESO 150-005 & $110_{-35}^{+45}$ & $0.150_{-0.033}^{+\ldots .033}$ & 7.8 \\
NGC 0115 & $130_{-29}^{+41}$ & $0.044_{-0.007}^{+0.010}$ & 3.9 \\
UGC 00260 & $430_{-288}^{+\ldots}$ & $0.070_{-0.012}^{+0.023}$ & 5.8 \\
$\ldots$ & & & \\
\hline
\end{tabular}

Notes. The $v_{\mathrm{c}}$ and spin are obtained from BP00.

a Same as the $\mathrm{S}^{4} \mathrm{G}$ nomenclature.

b Circular velocity (mode) $v_{\mathrm{c}}$ plus-minus $1 \sigma$ uncertainty in $\mathrm{km} \mathrm{s}^{-1}$.

${ }^{c}$ Spin parameter (mode) $\lambda$ plus $1 \sigma$ uncertainty.

d Numerical morphological type.

(This table is available in its entirety in machine-readable form.)

mode out of 987 have actual measurements in HyperLeda. In the case of the mode, we quantify the $1 \sigma(68.269 \%)$ distribution range to the left (right) of the mode by counting only the bins on the left-hand side (right-hand side) distribution starting from the bin of the mode but excluding it from the counts. Also, in case of multimodal distributions, we choose the bin with the smallest associated value. When we compare the two, we see that most of our values are larger than the observed ones but rarely above twice the observed rotational velocity. This effect comes partly from the accuracy of extracting the peak value over the skewed distributions of the circular velocities and spin parameters that we obtained from our method, as can be seen in Figure 18. When the distribution is skewed to the left, the mean is systematically larger than the median and the median is larger than the mode. It is vice versa when the skew is to the right: then the mean is smaller than the median and the median is smaller than the mode. This comes from our grid of models (Figure 15) and the sampling that we use to extract the best model. For given observational uncertainties and as the slope flattens out, higher circular velocity models that match the observations largely increase. The same holds true for larger values for the $y$ intercept (i.e., fainter): the higher the spin, the more models that match the observations. Hence, the sampling distributions show a tail toward larger circular velocities and spin parameters. Using either the median or the mode gives similar results. This is shown in Figure 18, where using the median yields a similar scatter as when using the mode.

Our values are consistent with the observed values within a factor of 0.5-2, especially if the very large uncertainties present are taken into account. The distributions given in Figure 16 provide powerful tools to test the predictions for numerical simulations of disks in a cosmological context.

\subsection{Color Gradient versus Circular Velocity, Spin, and Stellar Mass}

Finally, we compare the color gradients (the slopes) obtained in the (FUV - NUV), (FUV - [3.6]), and (NUV - [3.6]) color profiles (with a cutoff at $R / R 80=0.5$ but no cutoff in SB; see Table 6) against the mode circular velocities, mode spins that we derived with the method described in Section 5.1, and stellar masses calculated from the $3.6 \mu \mathrm{m} \mathrm{SB}$. This is shown in Figure 19. The panels showing the circular velocity and spin comprise 987 galaxies, whereas the panels showing the stellar mass comprise 1541 galaxies. For the mode circular velocities, a large scatter is seen, especially for low-mass systems, in all three colors. In the case of the mode spin parameters, the scatter is very much the same throughout the entire range of spins for all morphological types and all three colors. Then, the color gradient versus the stellar mass plots show a large scatter for low-mass galaxies with stellar mass of around $10^{8}-10^{9} M_{\odot}$. On average, there is a trend toward a more negative gradient as we move to larger masses, therefore indicating bluer outer disks. However, most low-mass galaxies and a nonnegligible fraction of massive galaxies show positive color gradients.

\section{Discussion}

We discuss the circular velocity and spin of galaxies in the local universe in Section 6.1. It is crucial to understand where this UV emission is coming from within the galaxies (Section 6.2). In this regard, an important point that needs to be addressed is the fact that the UV emission comes not only from newly born massive stars but also from evolved low-mass stars. We also discuss galaxy evolution and the effects of the environment on populating the Green Valley (Section 6.3).

\subsection{Circular Velocity and Spin of Galaxies in the Local Universe}

The results shown in Section 5 show that it is possible to derive (albeit with relatively large uncertainties in specific cases) the statistical distribution of the circular velocity (total mass) and spin (specific angular momentum) of galaxies from the analysis of deep near-infrared photometry of their disks. Besides, the fact that we can impose some simple criteria to isolate the disk component of the profiles makes this kind of analysis a very powerful tool for application to upcoming surface photometry data from LSST, EUCLID, or WFIRST.

Our analysis reveals that up to the current surface brightness detection limits (we note that all $\mathrm{S}^{4} \mathrm{G}$ galaxies are detected by Spitzer but many low surface brightness objects might still be missing from the catalogs), nearby galaxies show a wide distribution in spin with a maximum at $\lambda \sim 0.06$ and a relatively high fraction (24\%) of galaxies with $\lambda>0.08$ (see top right panel of Figure 16).

The comparison of these values with those derived for the SINGS sample (Kennicutt et al. 2003) by Muñoz-Mateos et al. (2011) using a similar method and a similar set of models indicates a larger number of high- $\lambda$ systems in our sample, in terms of the mean, median, and mode of the distribution. This is expected in the case of the SINGS sample, as this is biased toward high surface brightness systems with low angular momentum content relative to their mass. In addition, the SINGS sample (75 galaxies) was constructed to sample physical parameters (morphological type, luminosity, and FIR/optical luminosity ratio) and, therefore, is not representative in numbers of different kinds of galaxies. With respect to the predictions of semi-analytic models (e.g., Mo et al. 1998), we find a median value that is displaced toward larger spins (0.06) relative to recent simulations $(\sim 0.036$, quite 

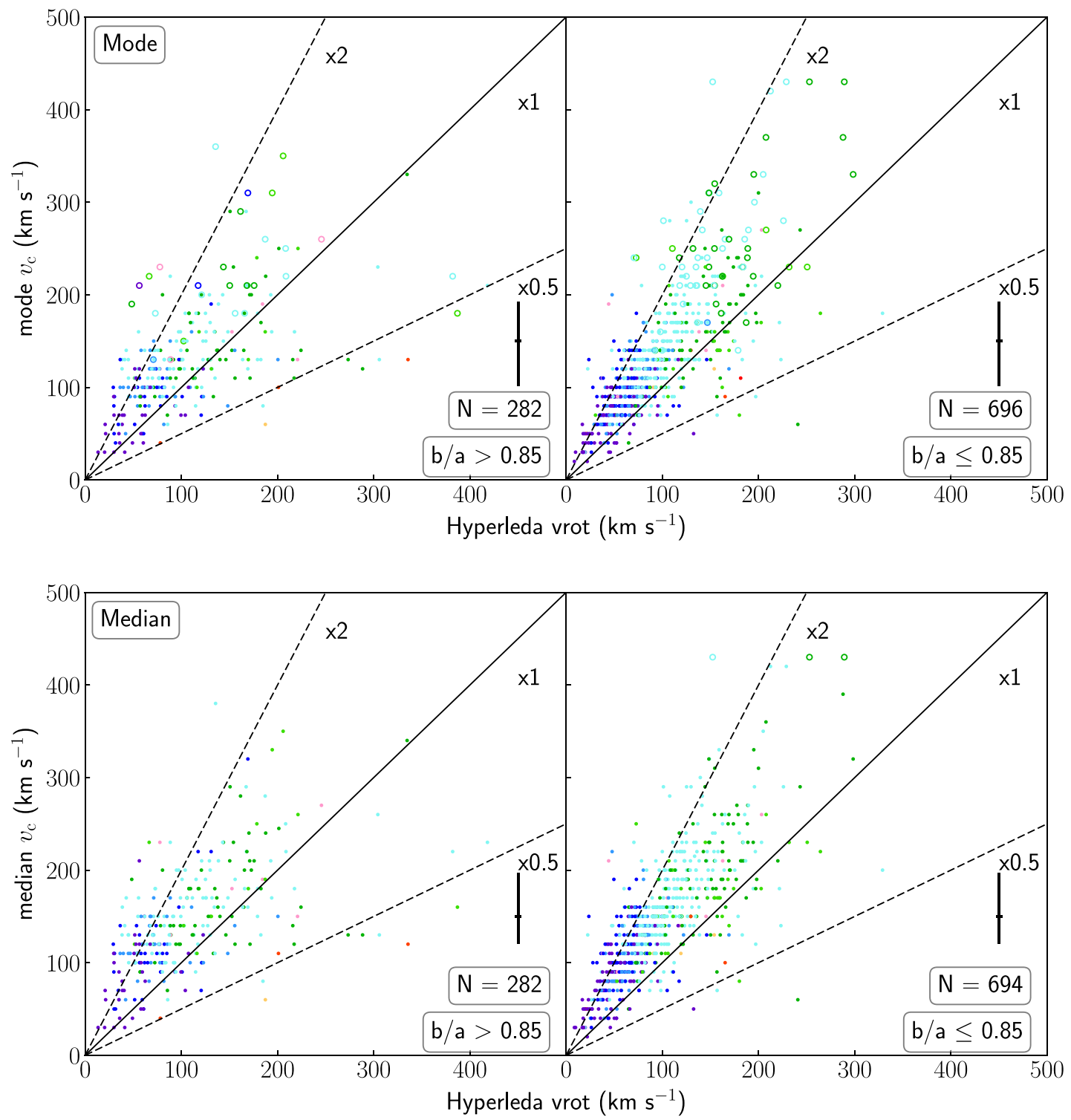

Figure 18. Rotational velocity $v_{\mathrm{c}}$ obtained from BP00 grid models for our sample galaxies once the $\mu_{[3.6]}$ profiles are corrected for inclination compared to the maximum rotational velocity obtained from HyperLeda (corrected for inclination) $v_{\text {rot }}$. The 1:1, 2:1, and 1:2 ratios are shown as dashed lines as visual guides. Here we use the mode (top) and median (bottom) of $v_{\mathrm{c}}$ obtained for each galaxy (see Figure 17). We distinguish between galaxies of high and low inclination using the minorto-major axis ratio $b / a(\leqslant 0.85$ for highly inclined galaxies in the right panel and $0.85<b / a \leqslant 1$ for low-inclination galaxies in the left panel).Both axes are in units of $\mathrm{km} \mathrm{s}^{-1}$. Open circles indicate galaxies that have extreme values, as in Figure 16, and for which we use their central value. We do not take their uncertainties into account for the computation of the average uncertainties.

independently of the galaxy mass and method of determining $\lambda$; Rodríguez-Puebla et al. 2016). To quantify this distribution, for the $\mathrm{S}^{4} \mathrm{G}$ sample, we derive mean (with $1 \sigma$ distribution width) and median values of $0.062 \pm 0.037$ and $0.054_{-0.024}^{+0.030}$, respectively, while for MW mass halos, the models of Rodríguez-Puebla et al. (2016) yield a mean of 0.036 with a dispersion of $0.24 \mathrm{dex}$ (for the spin parameter $\lambda_{P}$ of Peebles 1969).

With regard to the circular velocities, we find a relatively narrow mode distribution peaking at $120 \sim v_{\mathrm{c}} \sim 149 \mathrm{~km} \mathrm{~s}^{-1}$ (ignoring the outliers; top left panel of Figure 16). This indicates a lack of low-mass (dwarf) systems, which is probably occurring both at the high surface brightness (because of our diameter selection for $\mathrm{S}^{4} \mathrm{G}$ ) and low surface brightness (because of the limiting central surface brightness present in the catalogs of nearby galaxies) ends. Determining a volume- and diameter-corrected circular velocity (i.e., halo mass) function is beyond the scope of this paper. It could, however, be an interesting test for the models complementary to the halo mass functions derived from dynamical masses obtained from the modeling of $21 \mathrm{~cm}$ velocity maps and line profiles (de Blok et al. 2008; Papastergis et al. 2013; see also Zaritsky et al. 2014a, which connected the kinematics to the baryon fractions using the $\left.\mathrm{S}^{4} \mathrm{G}\right)$.

Finally, the distribution of circular velocity versus spin (bottom panel of Figure 16) shows a larger dependence of the spin on the circular velocity than that found by RodríguezPuebla et al. (2016). In those cases where a significant portion of the input probability distribution of the $y$ intercept and slope of the 1000 sampled points are outside of the grid, the mode is 


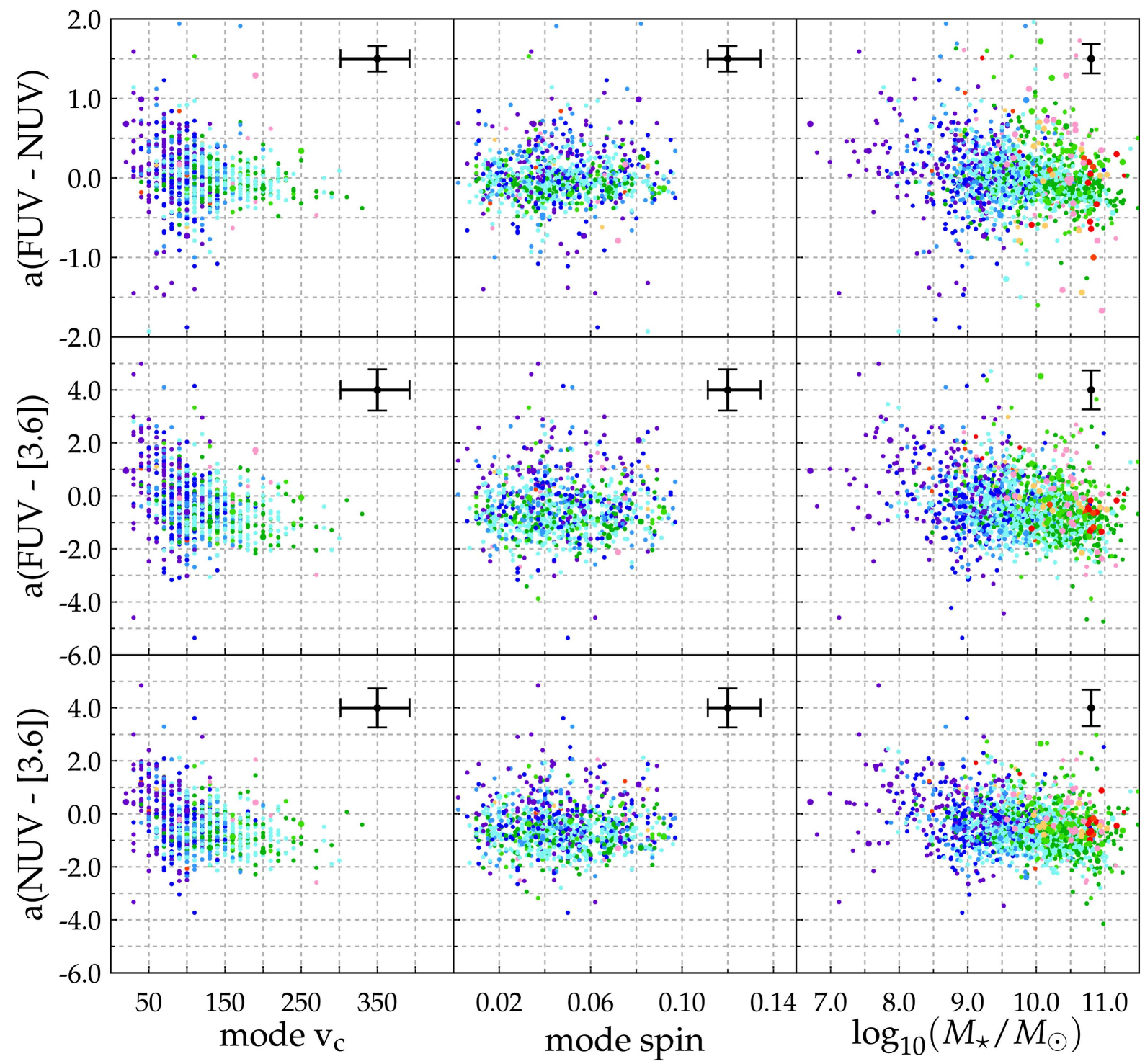

Figure 19. Color gradients (slopes of least-squares linear fit) in the (FUV - NUV), (FUV - [3.6]), and (NUV - [3.6]) colors vs. circular velocities (mode), spins (mode), and stellar masses, respectively. A positive color gradient indicates a reddening as we move to the outskirts. A negative color gradient indicates a bluing. Average $1 \sigma$ uncertainties in both axes are shown in the upper right corner of each panel.

biased toward the most extreme value model in spin or circular velocity, or both. We then use the central value (if the central value is within the grid) instead of the mode. If the central value is not in the grid, we ignore it. These are shown as open circles. The percentages are the fractions of outliers in circular velocity (top left) and spin parameter (bottom right). Outliers in both parameters are included in both fractions. Uncertainties for the outliers are not included in the average uncertainty. The lack of objects of high spin and low circular velocity could be due to the surface brightness limit involved in defining our sample. However, this would make our distribution even wider toward high- $\lambda$ values (see also Mo et al. 1998). Besides, the lack of low-mass, low-spin galaxies (high surface brightness dwarfs) is attributable to our diameter selection ( $\left.>1{ }^{\prime \prime}\right)$, as these would be very compact dwarf systems.

Thus, we conclude that the strong dependence of the spin on the circular velocity and, in particular, the lack of low-mass galaxies at both extremes of the distribution in spin might be due to selection effects in the $\mathrm{S}^{4} \mathrm{G}$ survey (at least, we cannot conclude otherwise). The relatively flat distribution of spin values in the range $\lambda=0.03-0.11$, which would be even more extended toward high- $\lambda$ values, used to pose a challenge to current models of galaxy formation. However, Amorisco \& Loeb (2016) recently showed that the properties and abundances in clusters of large, ultra-diffuse galaxies (UDGs) can be reproduced from within a standard cosmological framework and classical disk-formation models. It will be interesting to see, once catalogs of low surface brightness disk galaxies are available (including UDGs such as those found by Koda et al. 2015 in Coma), how they are distributed in terms of spin and circular velocity.

\subsection{Radial Distribution of UV Emission: UV Upturn and Star Formation}

The UV emission found in the 1931 galaxies within the $\mathrm{S}^{4} \mathrm{G}$ sample is clearly aligned in two sequences of UV-to-IR colors. These two sequences, which are called the GRS and GBS and are best isolated in the (FUV - NUV) and (NUV $-3.6 \mu \mathrm{m})$ 
color-color diagram and when galaxies are previously split by morphological type (see Figure 11), each correspond to a different mechanism responsible for the UV emission. In the case of the GRS, the big change in (FUV - NUV) color $(\sim 1.5 \mathrm{mag})$ with a change in (NUV $-3.6 \mu \mathrm{m})$ of $<1 \mathrm{mag}$ can only be attributed to the UV-upturn phenomenon (O'Connell 1999), which is believed to be caused by very hot EHB stars (see Zaritsky et al. 2015 and references therein).

On the other hand, the GBS has a slope of 0.12 (see Equation (1)), which implies a change of only $1.2 \mathrm{mag}$ in the (FUV - NUV) color for a change of $10 \mathrm{mag}$ in (NUV $-3.6 \mu \mathrm{m})$. As shown in Bouquin et al. (2015), this slope agrees well with the color correlation predicted by spectrophotometric models for the evolution of galaxy disks (see, e.g., BP00), so the UV emission of these objects can be interpreted as due to emission from relatively massive stars in the turnoff of the main sequence.

The GRS is mainly populated by E, E-S0, S0, and S0-a morphological types, and it is clearly isolated in its (FUV - NUV) blue end only in ETGs, i.e., E, E-S0, and S0 galaxies. This isolation is possible thanks to the dichotomy in the (NUV $-3.6 \mu \mathrm{m}$ ) colors of the central regions of ETGs (triangles in Figure 11). They are either very blue, indicating (residual) star formation in these innermost regions, or very red, which points toward a very old (light-weighted) stellar population. The fact that these very old populations in the centers of ETGs are also the ones showing the strongest UV upturn is something that has been explained in the past as being related to either the older age or higher metal (helium, at least) abundance of the horizontal-branch stars responsible for the UV emission in these regions (Boselli et al. 2005), but it may also be tied to the observations supporting differences in IMF (e.g., Cappellari et al. 2012, 2013a, 2013b; Conroy \& van Dokkum 2012).

In early-to-intermediate spirals ( $\mathrm{S} 0$-a through $\mathrm{Sc}$ ), the central regions of many galaxies appear in the locus where the GBS and GRS overlap. Besides, the entire GBS is well populated by measurements obtained in both the inner and outer regions of galaxies. Thus, these colors are of no use to determine whether the UV emission from the bulges of these galaxies is dominated by emission from young massive or evolved low-mass EHB stars or a combination of both. Both the outer parts of early-tointermediate spirals (except for regions populating the GGV; see below) and the late-type spirals ( $\mathrm{Sd}$ and beyond) at all radii follow a narrow GBS. According to the color profiles shown in Figure 3, the majority of the galaxies that are found to populate the GBS show negative color gradients, which is in agreement with the global scenario of inside-out formation of their disks. The study of the most extreme cases of inside-out disk formation will be the subject of a future communication. At the surface brightness levels reached by our data, we do not find clear signs of color upbending, at least in the bands considered in this work (see Bakos et al. 2008 and Marino et al. 2016 for studies of reversed optical color profiles and ionized-gas chemical abundance gradients in outer disks).

Finally, it is also worth mentioning that the central regions of galaxies (despite having the highest signal-to-noise ratios) show the widest dispersion in all three (FUV-NUV), (FUV $-3.6 \mu \mathrm{m}$ ), and (NUV $-3.6 \mu \mathrm{m}$ ) colors among galaxy types and, as a whole, covering $\sim 10 \mathrm{mag}$ in the case of the latter two colors. This, of course, indicates that nuclear regions are the least homogeneous within the population of local galaxies in terms of their stellar population and dust content.

\subsection{Galaxy Evolution through the Green Valley}

Here we focus on discussing the properties of those galaxies that were identified in Bouquin et al. (2015) as being globally included in the so-called GGV and of those regions within galaxies that are now found to be located in the GGV even though they are part of the GBS or GRS when considered as a whole.

In Figures 7 and 8, we showed that galaxies that belong (globally) to the GGV are mainly lenticulars and early-type spirals ( $\mathrm{S} 0-\mathrm{a}$ through $\mathrm{Sb}$ ) showing a relatively narrow distribution of (observed) sSFR around $10^{-12} \mathrm{yr}^{-1}$. Furthermore, Figure 9 shows that the outer regions of GGV galaxies behave differently from the outer regions of most GBS systems, with the FUV $-3.6 \mu \mathrm{m}$ color getting redder as we move progressively toward their outer disks. This clearly indicates that the reason these objects are in the GGV is that their disks are redder, for the same morphological type and surface brightness, than those of most GBS galaxies. Exploration of Figure 9 in the case of the GBS shows that the region of red disks is populated by a number of ETGs with profiles similar in shape to those found in the GRS, but that they probably show a very blue nucleus that places them in the GBS when considered as a whole.

A small fraction of GBS galaxies (mainly early-type spirals, but only a fraction of them) have disks that also redden with radius. These are objects that are likely to evolve into GGV galaxies or objects that GGV galaxies will evolve into, depending on whether GGV galaxies are quenching their star formation or regrowing a disk.

Our analysis shows that the fraction of galaxies belonging to dense environments is higher for GGV galaxies than for GBS galaxies but less than for the GRS. This result, combined with the fact that GGV galaxies have redder outer disks, hints at the direction of the evolution, from GBS to GRS, that favors star formation quenching due to environmental effects. Similar results have been obtained in the analysis of galaxies in clusters (see Bamford et al. 2009; Wolf et al. 2009; Cibinel et al. 2013; Head et al. 2014).

Moreover, a study of galaxies in transition in different environments (Vulcani et al. 2015) shows that galaxies in groups have a higher quenching efficiency than field galaxies. These results show that color transformation is due to the overall decrease in SFR, both in bulges and disks, while maintaining the morphology. They also show that morphological transformation is due to an increase in bulge-to-disk ratio because of disk removal, not because of the growth of the bulge, in disagreement with a bulge enhancement and absence of a disk-fading scenario (Christlein \& Zabludoff 2004). What is presented in Section 4 is in agreement with the former, where disk fading occurs, resulting in an increase in the bulge-to-disk ratio.

\section{Summary and Conclusions}

We have gathered GALEX FUV and NUV images for the $\mathrm{S}^{4} \mathrm{G}$ sample and measured their FUV and NUV magnitudes. Our UV subsample comprises 1931 galaxies and has an identical distribution in morphological type, distance, and $3.6 \mu \mathrm{m}$ absolute magnitude as the $\mathrm{S}^{4} \mathrm{G}$ sample of 2352 galaxies 
(Figure 1). Our GALEX subsample is compatible with being a random subsample of the entire $S^{4} \mathrm{G}$ sample and can also be considered representative of the local universe.

The photometry is done within rings with fixed PA and $\epsilon$ at 6 " steps in semimajor axis length and with a width of $6^{\prime \prime}$. The products are the $\mu_{\mathrm{FUV}}$ and $\mu_{\mathrm{NUV}}$ surface brightness profiles and (FUV - NUV) color profiles, along with the asymptotic FUV and NUV magnitudes and (FUV - NUV) color. Data are partially summarized in Table 1 , and the full catalog is available online. We have generated RGB postage-stamp images from UV images only and obtained the $\mu_{\mathrm{FUV}}, \mu_{\mathrm{NUV}}$ surface brightness, as well as the (FUV - NUV) color profiles. We used the RC2 numerical morphological classification to roughly classify the galaxies into narrower morphological type bins (sample demographics are summarized in Table 2).

These UV products, combined with the near-IR products of the $S^{4} \mathrm{G}$ sample, form an excellent set of tools to probe nearby galaxies, as we are directly tracing the current SFR with the former and the stellar mass with the latter, thus the sSFR. We have thus characterized the radial distributions of young and old stars in galaxies in the local universe.

We also looked at the spatially resolved colors formed by the three bands. The (FUV - NUV) color is most suitable for detecting variations in recent star formations on timescales below 1 Gyr. The (FUV - [3.6]) color is equivalent to a measurement of the observed sSFR. The (NUV - [3.6]) color is useful to construct the (FUV - NUV) versus (NUV - [3.6]) color-color diagram, in which the GBS and GRS subsamples are defined in the preliminary analysis of Bouquin et al. (2015). We see that the galaxies are grouped into narrow sequences in this color-color diagram and separate very well between starforming (GBS) and quiescent (GRS) galaxies. This allowed us to define an intermediate region, the GGV, where we find galaxies that are either leaving the blue sequence due to some damping of their star formation activity or leaving the red sequence, possibly by rejuvenation. We also perform the fit in the color profiles and show the distributions of the resulting slopes and $y$ intercepts (scale length and central SB of disks).

Our main results are the following.

1. The GBS, GGV, and GRS galaxies are well separated in the $\mu_{\mathrm{FUV}}$ versus $\mu_{[3.6]}$ plane. Most disks are located in a well-defined sequence that we call the "spatially resolved main sequence of star-forming disks," with $3.6 \mu \mathrm{m}$ surface brightness ranging from 20 to $25 \mathrm{mag} \operatorname{arcsec}^{-2}$ and FUV surface brightness ranging from 24 to $27 \mathrm{mag} \mathrm{arcsec}^{-2}$. The GBS galaxies are dominating the highest-surface sSFR densities, while the GRS galaxies are dominating the lowest-surface sSFR density. The ETGs of the GRS have a low surface sSFR density, $\Sigma_{\text {SSFR }}$, that stays radially constant at (or below) $10^{-12} \mathrm{yr}^{-1} \mathrm{pc}^{-2}$. The late-type galaxies of the GBS, on the other hand, have higher surface sSFR densities the later the type, with increasing surface sSFR density (bluing) inside-out. This is not always the case, since inside-out disk reddening is also seen for some of the galaxies. This reddening translates to sudden drops in surface SFR density and indicates a possible quenching (or damping; see Catalán-Torrecilla et al. 2017) of the star formation in the outskirts.

2. Star-forming GBS, quiescent GRS, and intermediate GGV galaxies are well separated in the (FUV - [3.6]) versus $\mu_{[3.6]}$ plane, especially when one looks at the colors of the isophote that encompasses $80 \%$ of the $3.6 \mu \mathrm{m}$ light (equivalent to the same percentage of stellar mass). The isophotes of the GGV galaxies fill the gap between the locus of the GBS and the GRS ones. Particularly, most GRS galaxies show very similar radial behavior to each other, and most of them end up in a similar locus in the (FUV - [3.6]) versus $\mu_{[3.6]}$ plane, where the $80 \%$ enclosed-light isophote ends up in a narrow range in $3.6 \mu \mathrm{m}$ surface brightness, between 21 and $23 \mathrm{mag} \operatorname{arcsec}^{-2}$, and in the (FUV - [3.6]) color range between 6 and 7 mag.

3. We performed an analysis of the $3.6 \mu \mathrm{m}$ surface brightness radial profiles by linearly fitting the data points using an array of cutoffs both in radial distance and in $3.6 \mu \mathrm{m}$ surface brightness to approximately exclude the bulge part and only fit the disk part. We find the best cutoffs values to be $R / R 80=0.5$ and $\mu_{[3.6]}=23.5$ mag $\operatorname{arcsec}^{-2}$ (corresponding to a stellar mass surface density of $3 \times 10^{7} M_{\odot} \mathrm{kpc}^{-2}$ ), where the mean reduced $\chi^{2}$ approaches unity and the number of galaxies is maximized $(>50 \%$ of the sample). Doing so, we efficiently exclude the bulge parts, as well as massive galaxies, and obtain a subsample of 987 disk galaxies for further analysis. This slope and $y$-intercept of the linear fit translate to circular velocities and central surface brightness (of the disk).

4. Finally, we compared the slope and $y$ intercept of the linear fit to the outer disk parts of our subsample to the slope and $y$ intercept of the linear fit to over 6258 simulated disk models of BP00 varying based on the circular velocity $v_{\mathrm{c}}$ and the spin parameter $\lambda$, thus obtaining a fine grid of slopes (i.e., scale length) and $y$ intercepts (i.e., central surface brightness). From this, we deduced the circular velocity for each of our galaxies by finding the closest model matching the slope and $y$ intercept of the galaxy. We find a distribution for the mode circular velocity with mean $v_{\mathrm{c}}=149 \pm 102$ $\mathrm{km} \mathrm{s}^{-1}$ (standard deviation $1 \sigma$ ) and median $v_{\mathrm{c}}=120_{-40}^{+70}$ $\mathrm{km} \mathrm{s}^{-1}$ ( $1 \sigma$ with the IQR method and thus excluding the outliers) and a distribution for the mode spin parameter with mean $\lambda=0.062 \pm 0.037$ (standard deviation $1 \sigma$ ) and median $\lambda=0.054_{-0.024}^{+0.030}$ ( $1 \sigma$ with the IQR method). For the spin, we recover the probability distribution function of Mo et al. (1998), whereas for the circular velocity, our distribution is skewed toward higher circular velocities than the ones obtained from HyperLeda. The low-mass Sd, Sm, and Irr galaxies seem to be more affected than larger spiral galaxies. Despite the large scatter, this method yields circular velocities similar to those observed within a range of factors of one to two for most galaxies.

We acknowledge financial support to the DAGAL network from the People Programme (Marie Curie Actions) of the European Union's Seventh Framework Programme FP7/ 2007-2013/under REA grant agreement number PITN-GA2011-289313. AYKB also thanks Professor Emeritus John E. Beckman for the valuable suggestions during private communications. JHK thanks the Astrophysical Research Institute of Liverpool John Moores University for their hospitality and the Spanish Ministry of Education, Culture and Sports for financial support of his visit there through 
grant number PR2015-00512. JHK acknowledges financial support from the Spanish Ministry of Economy and Competitiveness (MINECO) under grant numbers AYA2013-41243-P and AYA2016-76219-P. We acknowledge GALEXview and the STScI team for the data provided. We acknowledge the usage of the HyperLeda database (http://leda.univ-lyon1.fr). This research made use of Astropy, a community-developed core Python package for astronomy (Astropy Collaboration et al. 2013). We are grateful to our anonymous referee for the very constructive and excellent suggestions.

\section{Appendix A}

\section{A.1. Deriving $\Sigma_{\star}$ from $\mu_{[3.6]}$}

In this work, we make use of the stellar mass surface density $\Sigma_{\star}\left(M_{\odot} \mathrm{pc}^{-2}\right)$ distribution that is obtained from the $3.6 \mu \mathrm{m}$ surface brightness $\left(\mathrm{AB}\right.$ mag $\left.\operatorname{arcsec}^{-2}\right)$ radial profiles. We start from the definition of absolute magnitude,

$$
M_{[3.6], \star}=M_{[3.6], \odot}-2.5 \log _{10}\left(\frac{L_{[3.6], \star}}{L_{[3.6], \odot}}\right) \text {, }
$$

where $M_{[3.6], \star}$ and $L_{[3.6], \star}$ are the $3.6 \mu \mathrm{m}$ absolute magnitude (AB) and luminosity (in erg s ${ }^{-1} \mathrm{~Hz}^{-1}$ ) of the galaxy and $M_{[3.6], \odot}$ and $L_{[3.6], \odot}$ are the solar $3.6 \mu \mathrm{m}$ absolute magnitude and luminosity.

We also need the following expression for the mass-to-light ratio:

$$
\frac{M_{\star}}{L_{[3.6], \star}}=\Upsilon_{[3.6]},
$$

where the mass-to-light ratio of the $\operatorname{Sun}\left(M_{\odot} / L_{[3.6], \odot}\right)$ is unity and $M_{\star}$ is the stellar mass of the galaxy, $L_{[3.6], \star}$ is the luminosity at $3.6 \mu \mathrm{m}, M_{\odot}$ is solar mass, and $L_{\odot, 3.6}$ is the solar luminosity at $3.6 \mu \mathrm{m}$. Here $\Upsilon_{[3.6]}$ is the mass-to-light ratio at $3.6 \mu \mathrm{m}$ as obtained by Meidt et al. (2014) and is equal to 0.6 (assuming a Chabrier IMF).

Rearranging Equation (7), we have

$$
\frac{L_{[3.6], \star}}{L_{[3.6], \odot}}=10^{-0.4\left(M_{[3.6], \star}-M_{[3.6], \odot)} .\right.}
$$

Rearranging Equation (8) and adding the conversion factor $a_{\mathrm{IMF}}$ for the transformation from a Chabrier IMF (Chabrier 2003) to Kroupa IMF (Kroupa 2001), we get

$$
\frac{M_{\star}}{M_{\odot}}=\frac{L_{[3.6], \star}}{L_{[3.6], \odot}} \cdot \Upsilon_{[3.6]} \cdot a_{\mathrm{IMF}},
$$

where we use, in our case, $a_{\mathrm{IMF}}=\frac{M / L(\text { Kroupa })}{M / L(\text { Chabrier })}=1.034$ (conversion factors from Madau \& Dickinson 2014).

We then take the $\log$ of Equation (10), combined with Equation (9):

$$
\log _{10}\left(\frac{M_{\star}}{M_{\odot}}\right)=\log _{10}\left(\frac{L_{[3.6], \star}}{L_{[3.6], \odot}}\right)+\log _{10}\left(\Upsilon_{[3.6]} \cdot a_{\mathrm{IMF}}\right),
$$

i.e.,

$$
\log _{10}\left(\frac{M_{\star}}{M_{\odot}}\right)=0.4\left(M_{[3.6], \odot}-M_{[3.6], \star}\right)+\log _{10}\left(\Upsilon_{[3.6]} \cdot a_{\mathrm{IMF}}\right) .
$$

Finally, changing $M_{[3.6], \star}$ to $\mu_{[3.6]}\left(\mathrm{AB}\right.$ mag $\left.\operatorname{arcsec}^{-2}\right)+$ $5-5 \log _{10} d(\mathrm{pc})$ and converting $\operatorname{arcsec}^{-2}$ to $\mathrm{pc}^{-2}$ gives

$$
\begin{aligned}
\Sigma_{\star}\left(M_{\odot} \mathrm{pc}^{-2}\right)= & \Upsilon_{[3.6]} \cdot a_{\mathrm{IMF}} \cdot 10^{0.4 M_{[3.6], \odot}} \\
& \cdot 10^{-0.4 \cdot\left(\mu_{[3.6]}+5-5 \log _{10} d\right)} \cdot\left(\frac{206265}{d}\right)^{2},
\end{aligned}
$$

where $M_{[3.6], \odot}$ is the Sun's $3.6 \mu \mathrm{m}$ absolute $\mathrm{AB}$ magnitude, which is taken to be $6.03 \mathrm{mag}$ (converted to $\mathrm{AB}$ scale from the Vega magnitude value, $M_{\odot, 3.6 \text {, vega }}=3.24$, given by Equation (13) in Oh et al. 2008).

This corresponds to a stellar mass surface density $M_{\star} /$ area $=1.045 M_{\odot} \mathrm{pc}^{-2}$ at a $3.6 \mu \mathrm{m}$ surface brightness $\mu_{[3.6]}=27 \mathrm{mag} \mathrm{arcsec}^{-2}$ in the case of a Chabrier IMF and $M_{\star} /$ area $=1.080 M_{\odot} \mathrm{pc}^{-2}$ in the case of a Kroupa IMF. The equation, then, simplifies to the following:

$$
\log _{10}\left(\Sigma_{\star}\left(M_{\odot} \mathrm{pc}^{-2}\right)\right)=10.819-0.4 \mu_{[3.6]}+\log _{10} a_{\mathrm{IMF}} \text {, }
$$

where the term $\log _{10} a_{\mathrm{IMF}}=0$ for a Chabrier IMF, 0.015 for a Kroupa IMF, and 0.215 for a Salpeter IMF.

\section{Appendix B}

\section{B.1. Deriving the Observed SSFR from (FUV - [3.6])}

We start with the SFR (UV), assuming a Salpeter IMF (Salpeter 1955) and using a calibration by Madau et al. (1998), as provided by Kennicutt (1998), which we can convert to the expression for a Kroupa IMF by multiplying by $b_{\mathrm{IMF}}=0.67$ or by $b_{\mathrm{IMF}}=0.63$ for a Chabrier IMF, as reviewed and prescribed in Kennicutt \& Evans (2012) and Madau \& Dickinson (2014),

$$
\operatorname{SFR}\left(M_{\odot} \mathrm{yr}^{-1}\right)=1.4 \times 10^{-28} \cdot b_{\mathrm{IMF}} \cdot L_{\nu}\left(\mathrm{erg} \mathrm{s}^{-1} \mathrm{~Hz}^{-1}\right),
$$

and with the following expression of luminosity:

$$
\begin{aligned}
& L_{\nu}=4 \pi(d(\mathrm{~cm}))^{2} f_{\nu} \\
& L_{\nu}=4 \pi\left[d(\mathrm{pc}) \cdot 3.086 \times 10^{18}(\mathrm{~cm} / \mathrm{pc})\right]^{2} 10^{-0.4(\mathrm{FUV}+48.6)} .
\end{aligned}
$$

Combining the two equations above gives

$$
\begin{aligned}
\log _{10}(\mathrm{SFR})\left(M_{\odot} \mathrm{yr}^{-1}\right)= & 2 \log _{10} d(\mathrm{pc})-0.4 \mathrm{FUV} \\
& -9.216+\log _{10} b_{\mathrm{IMF}},
\end{aligned}
$$

where FUV is in $\mathrm{AB}$ magnitudes, the distance $d$ is in pc, and $\log _{10} b_{\mathrm{IMF}}=0,-0.174$, and -0.201 for a Salpeter, Kroupa, and Chabrier IMF, respectively.

Second, we need to introduce the distance modulus $m-M=5-5 \log _{10} d(\mathrm{pc})$ into Equation (12), so this becomes

$$
\begin{aligned}
\log _{10}\left(\frac{M_{\star}}{M_{\odot}}\right)= & \log _{10}\left(\Upsilon_{3.6} \cdot a_{\mathrm{IMF}}\right) \\
& -0.4\left([3.6]+5-5 \log _{10} d-6.03\right),
\end{aligned}
$$


where [3.6] is the apparent $\mathrm{AB}$ magnitude at $3.6 \mu \mathrm{m}, d$ is the distance in pc, and $M_{\odot, 3.6, \mathrm{AB}}=6.03$ (see Appendix A).

Finally, we combine Equations (17) and (18):

$$
\log _{10}(\mathrm{SSFR})=\log _{10}\left(\frac{\mathrm{SFR}}{M_{\star}}\right)=\log _{10}(\mathrm{SFR})-\log _{10}\left(M_{\star}\right) .
$$

It thus becomes

$$
\begin{aligned}
\log _{10}(\mathrm{sSFR})= & -0.4(\mathrm{FUV}-[3.6])-9.628+\log _{10} b_{\mathrm{IMF}} \\
& -\log _{10}\left(\Upsilon_{3.6} \cdot a_{\mathrm{IMF}}\right) .
\end{aligned}
$$

We can thus obtain the logarithm of the sSFR (in units of $\mathrm{yr}^{-1}$ ) from the (FUV - [3.6]) (AB mag) color. We emphasize that these quantities would not be corrected for extinction.

\section{ORCID iDs}

Alexandre Y. K. Bouquin (ib https://orcid.org/0000-00018285-4189

Armando Gil de Paz (iD https://orcid.org/0000-0001-6150-2854

Samuel Boissier (iD https://orcid.org/0000-0002-9091-2366

Kartik Sheth (iD https://orcid.org/0000-0002-5496-4118

Dennis Zaritsky (ID https://orcid.org/0000-0002-5177-727X

Reynier F. Peletier (i) https://orcid.org/0000-0001-7621-947X

Johan H. Knapen (iD https://orcid.org/0000-0003-1643-0024

Jesús Gallego (iD https://orcid.org/0000-0003-1439-7697

\section{References}

Amorisco, N. C., \& Loeb, A. 2016, MNRAS, 459, L51

Andrae, R., Schulze-Hartung, T., \& Melchior, P. 2010, arXiv:1012.3754

Astropy Collaboration, Robitaille, T. P., Tollerud, E. J., et al. 2013, A\&A, 558, A 33

Bakos, J., Trujillo, I., \& Pohlen, M. 2008, ApJL, 683, L103

Bamford, S. P., Nichol, R. C., Baldry, I. K., et al. 2009, MNRAS, 393, 1324

Boissier, S., \& Prantzos, N. 1999, MNRAS, 307, 857 (BP99)

Boissier, S., \& Prantzos, N. 2000, MNRAS, 312, 398 (BP00)

Boselli, A., Boissier, S., Cortese, L., et al. 2006, ApJ, 651, 811

Boselli, A., Cortese, L., Deharveng, J. M., et al. 2005, ApJL, 629, L29

Bouquin, A. Y. K., Gil de Paz, A., Boissier, S., et al. 2015, ApJL, 800, L19

Buta, R. J., Sheth, K., Athanassoula, E., et al. 2015, ApJS, 217, 32

Cano-Díaz, M., Sánchez, S. F., Zibetti, S., et al. 2016, ApJL, 821, L26

Cappellari, M., McDermid, R. M., Alatalo, K., et al. 2012, Natur, 484, 485

Cappellari, M., McDermid, R. M., Alatalo, K., et al. 2013a, MNRAS, 432, 1862

Cappellari, M., Scott, N., Alatalo, K., et al. 2013b, MNRAS, 432, 1709

Cardelli, J. A., Clayton, G. C., \& Mathis, J. S. 1989, ApJ, 345, 245

Catalán-Torrecilla, C., Gil de Paz, A., Castillo-Morales, A., et al. 2017, ApJ, 848,87

Chabrier, G. 2003, PASP, 115, 763

Christlein, D., \& Zabludoff, A. I. 2004, ApJ, 616, 192

Cibinel, A., Carollo, C. M., Lilly, S. J., et al. 2013, ApJ, 777, 116

Conroy, C., \& van Dokkum, P. G. 2012, ApJ, 760, 71

de Blok, W. J. G., Walter, F., Brinks, E., et al. 2008, AJ, 136, 2648 de Souza, R. E., Gadotti, D. A., \& dos Anjos, S. 2004, ApJS, 153, 411

Erroz-Ferrer, S., Knapen, J. H., Font, J., et al. 2012, MNRAS, 427, 2938

Erroz-Ferrer, S., Knapen, J. H., Leaman, R., et al. 2016, MNRAS, 458, 1199

Fang, J. J., Faber, S. M., Salim, S., Graves, G. J., \& Rich, R. M. 2012, ApJ, 761,23

Fazio, G. G., Hora, J. L., Allen, L. E., et al. 2004, ApJS, 154, 10

Freeman, K. C. 1970, ApJ, 160, 811

French, K. D., Yang, Y., Zabludoff, A., et al. 2015, ApJ, 801, 1

Gadotti, D. A. 2008, MNRAS, 384, 420

Gavazzi, G., Boselli, A., Donati, A., Franzetti, P., \& Scodeggio, M. 2003 , A\&A, 400, 451

Gil de Paz, A., Boissier, S., Madore, B. F., et al. 2007, ApJS, 173, 185

Gil de Paz, A., Madore, B. F., Boissier, S., et al. 2005, ApJL, 627, L29
González Delgado, R. M., Cid Fernandes, R., García-Benito, R., et al. 2014, ApJL, 791, L16

Graham, A. W., \& Worley, C. C. 2008, MNRAS, 388, 1708

Head, J. T. C. G., Lucey, J. R., Hudson, M. J., \& Smith, R. J. 2014, MNRAS, 440,1690

Herrera-Endoqui, M., Díaz-García, S., Laurikainen, E., \& Salo, H. 2015, A\&A, 582, A86

Kauffmann, G., Heckman, T. M., De Lucia, G., et al. 2006, MNRAS, 367, 1394

Kawata, D., \& Mulchaey, J. S. 2008, ApJL, 672, L103

Kennicutt, R. C., \& Evans, N. J. 2012, ARA\&A, 50, 531

Kennicutt, R. C., Jr. 1998, ARA\&A, 36, 189

Kennicutt, R. C., Jr., Armus, L., Bendo, G., et al. 2003, PASP, 115, 928

Kim, T., Gadotti, D. A., Sheth, K., et al. 2016, yCat, 178

Knapen, J. H., Stedman, S., Bramich, D. M., Folkes, S. L., \& Bradley, T. R. 2004, A\&A, 426, 1135

Koda, J., Yagi, M., Yamanoi, H., \& Komiyama, Y. 2015, ApJL, 807, L2

Kroupa, P. 2001, MNRAS, 322, 231

Madau, P., \& Dickinson, M. 2014, ARA\&A, 52, 415

Madau, P., Pozzetti, L., \& Dickinson, M. 1998, ApJ, 498, 106

Makarov, D., Prugniel, P., Terekhova, N., Courtois, H., \& Vauglin, I. 2014, A\&A, 570, A13

Marino, R. A., Gil de Paz, A., Sánchez, S. F., et al. 2016, A\&A, 585, A47

Martin, D. C., Fanson, J., Schiminovich, D., et al. 2005, ApJL, 619, L1

Martin, D. C., Wyder, T. K., Schiminovich, D., et al. 2007, ApJS, 173, 342

Meidt, S. E., Schinnerer, E., van de Ven, G., et al. 2014, ApJ, 788, 144

Mo, H. J., Mao, S., \& White, S. D. M. 1998, MNRAS, 295, 319

Muñoz-Mateos, J. C., Boissier, S., Gil de Paz, A., et al. 2011, ApJ, 731, 10

Muñoz-Mateos, J. C., Gil de Paz, A., Boissier, S., et al. 2007, ApJ, 658 1006

Muñoz-Mateos, J. C., Gil de Paz, A., Boissier, S., et al. 2009a, ApJ, 701, 1965

Muñoz-Mateos, J. C., Gil de Paz, A., Zamorano, J., et al. 2009b, ApJ, 703, 1569

Muñoz-Mateos, J. C., Sheth, K., Regan, M., et al. 2015, ApJS, 219, 3

Norton, S. A., Gebhardt, K., Zabludoff, A. I., \& Zaritsky, D. 2001, ApJ, 557,150

Ochsenbein, F., Bauer, P., \& Marcout, J. 2000, A\&AS, 143, 23

O'Connell, R. W. 1999, ARA\&A, 37, 603

Oh, S.-H., de Blok, W. J. G., Walter, F., Brinks, E., \& Kennicutt, R. C., Jr. 2008, AJ, 136, 2761

Papastergis, E., Giovanelli, R., Haynes, M. P., Rodríguez-Puebla, A., \& Jones, M. G. 2013, ApJ, 776, 43

Paturel, G., Theureau, G., Bottinelli, L., et al. 2003, A\&A, 412, 57

Peebles, P. J. E. 1969, ApJ, 155, 393

Pezzulli, G., Fraternali, F., Boissier, S., \& Muñoz-Mateos, J. C. 2015, MNRAS, 451, 2324

Ponomareva, A. A., Verheijen, M. A. W., \& Bosma, A. 2016, MNRAS, 463, 4052

Querejeta, M., Meidt, S. E., Schinnerer, E., et al. 2015, ApJS, 219, 5

Rodríguez-Puebla, A., Behroozi, P., Primack, J., et al. 2016, MNRAS, 462,893

Salim, S., Fang, J. J., Rich, R. M., Faber, S. M., \& Thilker, D. A. 2012, ApJ, 755,105

Salo, H., Laurikainen, E., Laine, J., et al. 2015, ApJS, 219, 4

Salpeter, E. E. 1955, ApJ, 121, 161

Schawinski, K., Urry, C. M., Simmons, B. D., et al. 2014, MNRAS, 440 889

Schlegel, D. J., Finkbeiner, D. P., \& Davis, M. 1998, ApJ, 500, 525

Sheth, K., Regan, M., Hinz, J. L., Gil de Paz, A., \& Menéndez-Delmestre, K. 2010, PASP, 122, 1397

Thilker, D. A., Bianchi, L., Boissier, S., et al. 2005, ApJL, 619, L79

Thilker, D. A., Bianchi, L., Meurer, G., et al. 2007, ApJS, 173, 538

Tully, R. B., Courtois, H. M., Dolphin, A. E., et al. 2013, AJ, 146, 86

Vulcani, B., Poggianti, B. M., Fritz, J., et al. 2015, ApJ, 798, 52

Wolf, C., Aragón-Salamanca, A., Balogh, M., et al. 2009, MNRAS, 393, 1302

Wyder, T. K., Martin, D. C., Schiminovich, D., et al. 2007, ApJS, 173, 293

Yi, S. K., Lee, J., Sheen, Y.-K., et al. 2011, ApJS, 195, 22

Yi, S. K., Yoon, S.-J., Kaviraj, S., et al. 2005, ApJL, 619, L111

Yıldı, M. K., Serra, P., Peletier, R. F., Oosterloo, T. A., \& Duc, P.-A. 2017, MNRAS, 464, 329

Zaritsky, D., Courtois, H., Muñoz-Mateos, J.-C., et al. 2014a, AJ, 147, 134

Zaritsky, D., Gil de Paz, A., \& Bouquin, A. Y. K. 2014b, ApJL, 780, L1

Zaritsky, D., Gil de Paz, A., \& Bouquin, A. Y. K. 2015, MNRAS, 446, 2030 